\title{
15th International Nutrition and Diagnostics Conference INDC 2015
}

\author{
Prague, Czech Republic, October 5-8, 2015
}

Chairman: A. Horna

Scientific Organising Committee: I. Acworth, I. Bondarenko, J. Ehrenbergerová, A. Horna, P. Jandera, E. Jansen, R. Mabrouk, M. Macka, M. Šamánek, H. Tlaskalová-Hogenová, J. Xiao, Z. Zadák, T. Zima

Local Organising Committee: J. Blattná, A. Horna, J. Hornová, M. Hornová, J. Vávrová, Š. Voborníková, C. Garnica Ortiz

\section{Abstracts for Plenary Sessions}

Diabetes, nutrition and oxidative stress

\section{Jansen $E^{\star}$}

Centre for Health Protection, National Institute for Public Health and the Environment, PO Box 1, 3720 BA Bilthoven, the Netherlands, E-mail: eugene.jansen@rivm.nl

Diabetes Mellitus is a chronic disease which is characterized by a decreased ability to utilize glucose as an energy source. As a result high serum glucose levels will occur without a medical treatment by insulin injections (type 1 diabetes) or oral drugs (type 2 diabetes). Less attention is paid to the influence of nutrition. Patients with diabetes often show an increased level of inflammation and/or oxidative stress and symptoms of metabolic syndrome, such as abdominal obesity, elevated blood pressure, elevated plasma glucose, high serum triglycerides, and low HDL cholesterol. Nutrition cannot cure diabetes, but can contribute to a (partial) prevention of adverse symptoms connected to the disease.

In this paper several aspects in nutrition will be discussed, such as a high glycemic diet (glucose, fructose), a diet causing inflammation (high caloric, iron) and oxidative stress, consumption of different fats (saturated, omega 3/6 ratio), antioxidants in the diet (fruits and vegetables) and the use of supplements such as multivitamins, single vitamins (E, D, K2) and lipoic acid. Examples will be given from research and own experience.

The role of isotypes and molecular properties of antibodies to environmental antigens in health and disease

\section{Mestecky J}

University of Alabama at Birmingham, USA; Institute of Microbiology, Czech Academy of Sciences; and Institute of Immunology and Microbiology, 1st School of Medicine, Charles University, Prague, Czech Republic, E-mail: mestecky@uab.edu

Environmental antigens from ingested food, inhaled air, and commensal microbiota resident on mucosal surfaces stimulate the entire immune system including the induction of systemic and mucosal immune responses. In healthy individuals humoral responses in external secretions are represented by antibodies dominantly of the IgA isotype which display the protective activities by inhibition of penetration of foreign antigens. Due to its anti-inflammatory activity
IgA effectively inhibits potential tissue damage induced by antibodies of other isotypes. This may not be the case in individuals with limited IgA responses. In a number of diseases including IgA deficiency, infection with the human immunodeficiency virus, type I hypersensitivity and others, the absence or restricted humoral response in the IgA isotype may lead to the induction of antibodies of other isotypes (e.g., IgG or IgE) with pathologic consequences. These include the formation of immune complexes capable of activation of complement cascade and their deposition with ensuing damage in target tissues. Furthermore, the frequent alteration of glycan moieties associated with certain Ig isotypes may further accentuate their inflammatory potential. Consequently, the isotype as well as molecular aberrancies of molecules of antibodies specific for environmental antigens play an important role in the protection or potential tissue damage.

The birth of a microbiome: changes to the microbiota during pregnancy

\section{Koren $\mathbf{O}^{*}$}

Faculty of Medicine, Bar-Ilan University, Israel,E-mail:korenomry@ gmail.com

During pregnancy the female body undergoes hormonal, metabolic, and immunological changes such as an increase in body fat early in pregnancy followed by reduced insulin sensitivity later in gestation. Pregnancy progression is also associated with dramatic alterations in the composition of the gut and vaginal microbiotas. The vaginal microbiota of pregnant women is characterized with a decrease in bacterial diversity which is also seen in the gut microbiota as pregnancy progresses. In the gut, the lower diversity is accompanied by an increase in "between sample" diversity and an increase in the number of Proteobacteria and opportunistic pathogens. Germfree mice inoculated with gut microbiota from pregnant women presented metabolic changes mirroring those of the pregnant women. We are now at the point of trying to understand whether these changes in community structure are a cause or consequence of some of the

Received: May 25, 2016; Accepted: September 15, 2016; Published: September 23, 2016 
characteristics of pregnancy. It will be interesting to determine whether pregnancy associated microbiota alterations are required for a healthy pregnancy and whether pregnancy complications such as gestational diabetes are associated with dysbiosis?

\section{The mucosal immune system and its exploitation by mucosal vaccines}

\section{Russell M*}

University at Buffalo, Buffalo, NY, USA, E-mail: russellm@buffalo.edu

Most infectious diseases directly afflict or are acquired through the mucosal surfaces of the body consisting of the oro-gastrointestinal, respiratory, and genital tracts, the conjunctivae, their associated glands and the mammary glands. These extensive surfaces are protected by the mucosal immune system, which represents the largest compartment of the entire immune system. Numerous innate defense mechanisms include physicochemical barriers and a variety of anti-microbial proteins secreted by epithelial cells, as well as innate myeloid and lymphoid cells. The generation of adaptive immune responses at mucosal surfaces, including specific secretory IgA antibodies and effector $\mathrm{T}$ cells of various types requires stimulation of the mucosal immune system through specialized inductive sites which disseminate activated lymphocytes to nearby and remote mucosal effector sites. However, relatively few mucosally delivered vaccines have so far been developed for human use. A major function of the mucosal immune system is to control the commensal microbiota without eliminating it, and recent findings are revealing the importance of the microbiota in human health. The mucosal immune system must therefore critically distinguish not only between self and non-self antigens, but also between harmless food materials that must be tolerated, commensals that must be accommodated, and pathogens and their toxins that must be eliminated. The last function must be accomplished without inflicting damage on the mucosal epithelia, which stand as the first barrier against invasion. The default mode of mucosal immune response is therefore hyporereactive, non-inflammatory, and of limited duration. Mucosal vaccination strategies have been designed to overcome these limitations, and include a variety of delivery systems designed to protect vaccine antigens against digestion and ensure their uptake by inductive site tissues, as well as novel adjuvants to elicit the desired types of response.

\section{DNA damage, DNA repair and colorectal cancer}

Vodička $\mathrm{P}^{1,2^{+}}$, Slyskova $\mathrm{J}^{1,2}$, Pardini $\mathrm{B}^{3}$, Svoboda $\mathrm{M}^{1,2}$, Vodickova $\mathrm{L}^{1,2}$, Naccarati $A^{1,3}$ and Hemminki $K^{4}$

${ }^{1}$ Department ofMolecular Biology of Cancer, Institute of Experimental Medicine, Academy of Science of the Czech Republic, Prague, Czech Republic

${ }^{2}$ Institute of Biology and Medical Genetics, First Medical Faculty of Charles University, Prague, Czech Republic

${ }^{3}$ Human Genetics Foundation, Torino, Italy

${ }^{4}$ Department ofMolecular Genetics Epidemiology, German Cancer Research Center (DKFZ), Heidelberg, Germany, E-mail: pvodicka@ biomed.cas.cz

Introduction: DNA repair maintains universal genomic stability and preserves cellular functions. Defective DNA repair predisposes individuals to cancer by accumulating DNA damage and mutations. Measurement of DNA repair capacity (DRC) through functional assays integrates effects of epigenetic factors, genetic polymorphisms, gene expression, stability of gene product, effect of inhibitors/stimulators, environmental and lifestyle factors.

Methods: We recently developed functional assays to measure nucleotide excision (NER) and base excision (BER) repair capacities in human tissues (1). MiRNA, lncRNA and DNA repair transcript levels were analysed by RT PCR (Biomark II Fluidigm platform), protein expressions by western blotting and immunohistochemistry, activities of SMUG1 and UNG1,2 by fluorimetric assay. SNPs were determined by KASP genotyping, their functional relevance by a luciferase reporter assay.

Results: Healthy subjects exposed to chemical carcinogens exhibited decreased DNA damage and increased BER capacity. On the contrary, DRC were significantly lower and DNA damage higher in incident colorectal cancer (CRC) patients than in matched control subjects. In CRC patients the DRC, significantly lower at the time of diagnosis, increased to the levels observed in healthy controls following the completion of chemotherapy (2). We measured DRC in blood cells, healthy mucosa and tumor tissues from 70 patients with sporadic CRC. Lymphocytes in contrast to tumor tissues exhibited lowest DRC (1). SNPs in miRNA binding sites of SMUG1 affected significantly survival in 5-fluorouracil-treated CRC patients (3). Further mechanistic investigation comprises transcript levels, protein expressions as well as uracil glycosylase activities in cell lysate (4).

Discussion/Evaluation: Our data indicate clearly alterations in excision DNA repair kinetics in CRC patients. However, the DRC should be determined in particular histo-pathological subtypes separately as well as in precancerous adenomas. Mechanistic investigations are undertaken on BER, SMUG1 and UNG1,2 glycosylases in particular, in relation to 5 -fluorouracil-treated CRC. The data are being evaluated regarding post-transcriptional regulation of DNA repair by miRNA and lncRNA, as suggested by our recent report (3).

Conclusion: Our data on DRC in modulating the chemotherapy outcome are quite promissing. However, the analysis of sequential steps from subjects in risk to cancer patients may further elucidate the prognostic value of DNA excision repair.

Kiwifruit-based carbohydrate exchanges effectively reduce the glycaemic impact of starch-based breakfast cereal by a combination of processes

\section{Monro $\mathrm{J}^{1 \star}$, Mishra $\mathrm{S}^{1}$, Edwards $\mathrm{H}^{2}$, Podd $\mathrm{J}^{2}$ and Ansell $\mathrm{J}^{3}$ \\ ${ }^{1}$ New Zealand Institute for Plant \& Food Research Limited, Palmerston North, New Zealand \\ ${ }^{2}$ Massey University, Palmerston North, New Zealand \\ ${ }^{3}$ Zespri International Limited, Tauranga, New Zealand, E-mail: john.monro@plantandfood.co.nz}

During digestion, kiwifruit tissues released from the physical constraint of the fruit may expand several-fold to a greater hydrated solids volume than the original fruit, and occupy available space in the foregut, surrounding and interacting with other food components. Inside this cell wall dispersion, in vitro digestion of available carbohydrate (CHOavail), diffusion of digestion products, and mixing responsible for mass transfer of nutrients to the gut wall for absorption, may be retarded, together reducing the glycaemic response to carbohydrate foods such as breakfast cereal consumed with kiwifruit. To measure the role of non-CHOavail components of kiwifruit in 
reducing the glycaemic response to co-consumed wheat cereal we formulated meals containing equal amounts of breakfast cereal, with the addition of either kiwifruit, or sugars of the same composition and quantity as in kiwifruit, so the only difference between the meals was the non-CHOavail kiwifruit components. The meal containing entire kiwifruit significantly reduced the glycaemic response amplitude $(\mathrm{p}<0.001)$ and the area under the $0-120 \mathrm{~min}$ incremental blood glucose response curve (IAUC) $(\mathrm{p}<0.022)$, each by $20 \%$ compared with the equi-carbohydrate diet containing the added kiwifruit sugars. It also significantly increased the 120-180 min IAUC by preventing postprandial overcompensation (cereal; -17.1 mmol.l-1l-1, cereal + kiwifruit, 17.3 mmol.l-1l-1, LSD 16.1, p<0.001), indicating improved homeostatic blood glucose control, and reduced hunger experienced at $180 \mathrm{~min}$. In a subsequent study, kiwifruit was included in a carbohydrate exchange format, kiwifruit partially replacing breakfast cereal in equi- carbohydrate meals. Blood glucose was further reduced $(25 \%$, $\mathrm{p}<0.001$ ), the satiating effect of the meals was not significantly reduced by kiwifruit substitution, and insulin demand was decreased. Whole kiwifruit may therefore be a valuable component in equi-carbohydrate diets for managing glycaemia, the multiple nutritional benefits of the whole fruit being provided with the additional functional benefit of improved glycaemic control.

Cut-offs of diagnostic indices in iron deficiency and irondeficiency anemia in Chinese infants

Lichen $\mathrm{Y}^{1,4^{*}}$, Xiaoguang $\mathrm{Y}^{1}$, Jie $\mathrm{L}^{2}$, Wei $\mathrm{Z}^{3}$, Jun $\mathrm{W}^{4}$, Yuejiao $\mathrm{W}^{2,3}$ and $\mathrm{Na} \mathrm{L}^{4}$

${ }^{1}$ National Institute of Nutrition and Health, China CDC

${ }^{2}$ Department of Pediatrics, The People's Hospital of Beijing University, China

${ }^{3}$ Department of Pediatrics, Beijing Maternity Hospital Affiliated to Capital Medical University, China

${ }^{4}$ Department of Obstetrics, The People's Hospital of Beijing University, China, E-mail: yanglichen28@126.com

Iron deficiency anemia (IDA) is a major public health problem in infants. Yet diagnostic criteria for iron deficiency (ID) and IDA in infants are poorly defined. Our study aimed to establish appropriate cut-off values for diagnosis of ID and IDA in 4- and 6-month-old Chinese infants. Pregnant women delivering at 37 or more gestational weeks with normal iron status were recruited after obtaining informed consent. Later, infants with normal birth weight were selected. Those who were not exclusively breastfed in the first 4 months were excluded from the study. At 4 months old, all infants were randomly assigned to a control group or an iron supplement group. The latter group was given a daily dose of iron supplement corresponding to $1 \mathrm{mg} / \mathrm{kg}$ body weight of elemental iron from 4 to 6 months of age. Before and at the end of the 2-month study period, vein blood samples was collected to analyze hemoglobin ( $\mathrm{Hb}$ ), mean corpuscular volume (MCV), serum ferritin(SF), soluble transferrin receptor (sTfR), and free erythrocyte protoporphyrin (FEP) levels. Individuals whose $\mathrm{Hb}$ response increased sharply after iron supplementation were excluded for possible ID. Cutoffs of all iron indices were determined as the limit of $95 \%$ confidence interval (CI) of the normal iron status infants enrolled in our study. A total of 309infants at 4 months and 136infants at 6 months were finally included in our statistical analysis. Our study suggests the following 2 SD cut-off values for the diagnosis of IDA in 4- and 6-month-old Chinese infants: SF6mg/L, Hb205 $\mu \mathrm{g} / \mathrm{dL}$ at 4 months; SF6mg/L, $\mathrm{Hb} 209 \mu \mathrm{g} / \mathrm{dL}$ at 6 months. The Chinese clinical trial registry number is

\section{ChiCTR-TRC-12002838.}

Does a low fodmap diet reduce symptoms associated with functional gastrointestinal disorders? - a meta-analysis

\section{Marsh A*, Eslick E and Eslick G}

The Whiteley-Martin Research Centre, Discipline of Surgery, The Sydney Medical School Nepean, Penrith, New South Wales, Australia, E-mail: abigail.marsh@uq.net.au

Introduction: Functional gastrointestinal symptoms such as abdominal pain, bloating, constipation, diarrhea and flatulence are common in patients with Irritable Bowel Syndrome (IBS) or Inflammatory Bowel Disease (IBD). The diversity of symptoms presenting has meant finding an effective treatment challenging with most treatments alleviating only the primary symptom. A novel treatment option for IBS and IBD currently generating much excitement is the low FODMAP (Fermentable, Oligo-, Di-, Monosaccharides And Polyols) diet.

Objective: To determine the evidence of the efficacy of a low FODMAP diet in the treatment of such symptoms.

Methods: Electronic databases were searched. Pooled odds ratios (ORs) and 95\% confidence intervals were calculated for the effect of a low FODMAP diet in the reduction of IBS Symptom Severity Score (SSS) and increase in IBS Quality of Life (QOL) score for both randomized clinical trials (RCTs) and non-randomized interventions using a random-effects model.

Results: Six RCTs and 16 non-randomized interventions were included in the analysis. There was a significant decrease in IBS SSS scores for those individuals on a low FODMAP diet in both the RCTs (OR: $0.44,95 \%$ CI: $0.25-0.76$ ) and non-randomized interventions (OR: 0.03 , 95\% CI: 0.01-0.20). In addition, there was a significant improvement in the IBS QOL score for RCTs (OR: 1.84, 95\% CI: 1.12-3.03) and for non-randomized interventions (OR: 3.18, 95\% CI: 1.60-6.31). Moreover, following a low FODMAP diet was found to significantly reduce symptom severity for abdominal pain (OR: 1.81, 95\% CI: 1.13-2.88), bloating (OR: 1.75, 95\% CI: 1.07-2.87) and overall symptoms (OR: 1.81, 95\% CI: 1.11-2.95) in the RCTs. In the nonrandomized interventions similar findings were observed.

Conclusion: This meta-analysis supports the efficacy of a low FODMAP diet in the treatment of functional gastrointestinal symptoms. Further research should ensure studies include an assessment of dietary adherence as part of a low FODMAP diet.

Effects of red grape polyphenols on the immune system in experimental models and humans

\section{Jirillo $E^{*}$}

Department of Basic Medical Sciences Neuroscience and Sensory Organs, University of Bari, Bari, Italy, E-mail: emilio.jirillo@uniba.it

Polyphenols are widely distributed in the vegetal kingdom and endowed with anti-oxidant and anti-inflammatory activities. Our own studies have been based on the use of polyphenols extracted from red grape belonging to various cultivars. As animal models, we have utilized mice with experimental colitis and farmed sea basses. Colitis mice have been administered for one week with fermented grape marc (FGM) from Koshu cultivar and compared to untreated animals and healthy controls. In untreated colitis mice, a dramatic reduction of the bowel length was observed which was partially prevented by the 
administration of polyphenols. In addition, in untreated sick animals levels of Tumor Necrosis Factor alpha and Interleukin(IL)-1 beta in colon homogenates were more elevated than in healthy mice, while polyphenol treatment dramatically reduced both cytokines. With regard to the fish model, farmed sea basses received a feed enriched in polyphenols (extracted from the seeds of Nero di Troia cultivar) over a period of six months and compared to fish fed a conventional feed. This dietary regimen led to a reduction of intestinal and splenic inflammatory cytokines and increase in Interferon (IFN) - gamma in comparison to conventionally fed fish. In both models, polyphenols exhibited anti-inflammatory and immunomodulating activities. In the human model, we have carried out both in vitro and in vivo studies. In vitro, healthy human peripheral lymphomonocytes when exposed to FGM from Negroamaro cultivar and Koshu cultivar released an array of inflammatory and anti-inflammatory cytokines. In particular, IL-10 (a typical anti-inflammatory cytokine) was released by $\mathrm{T}$ regulatory cells, as assessed in cytofluorimetric studies. In vivo, administration of Leucoselect-Phytosome (a compound enriched in polyphenols from red grape seeds) to frail elderly patients for one month led to a recovery of T helper 1 functions in terms of release of IFN-gamma and IL-2. Also these data strongly indicate the anti-inflammatory and immunomodulating activities of polyphenols from red grapes.

\section{Polysaccharides - a sweet part of probiotics}

\section{Górska ${ }^{\star}$, Brzozowska E and Gamian A}

Institute of Immunology and Experimental Therapy, Polish Academy of Sciences, Weigla 12, 53-114 Wrocław, Poland, E-mail:sabina.gorska@ iitd.pan.wroc.pl

Human bodies are a habitat for 10-100 trillion of microorganisms such as bacteria, viruses and eukaryotes and collectively this complex community constitutes our microbiota. Accumulating evidence indicates that especially the intestinal microbiota plays a major role in health and disease in humans: regulate our physiology and metabolism and, perhaps more importantly, provides signals for proper development of intestinal as well systemic immune compartments. Probiotics are live microorganisms that provide health benefits to the host when ingested in adequate amounts. The strains most frequently used as probiotics include lactic acid bacteria and bifidobacteria. Probiotics have demonstrated significant potential as therapeutic options for a variety of diseases, but the mechanisms responsible for these effects have not been fully elucidated yet. Several important mechanisms underlying the effects of probiotics include the following: modification of the gut microbiota, competitive adherence to the mucosa and epithelium, strengthening of the gut epithelial barrier and modulation of the immune system to convey an advantage to the host. At the intestinal epithelial level, bacteria and antigens are sampled by dendritic cells and subsequently presented to nave T cells in the Peyer's patches and mesenteric lymph nodes. Recognition of microbe-associated molecular patterns is known to be mediated by pattern recognition receptors that signal the presence of specific microorganisms to the host. Still very little is known about the bacterial effector molecules involved in bacteriahost crosstalk and very important questions remain largely without answer: Which biomolecule is responsible for immune activation? Lactobacillus cell envelope contains several effector molecules that are pivotal in the direct signaling capacity of these bacteria that underlies their immunomodulatory effects, including lipoteichoic acid, peptidoglycan, and (glyco)proteins, teichoic acid or polysaccharides (PSs) that may not be involved in direct signaling to the host cell, but still affect signaling through shielding of other bacterial effector molecules.
Bacterial PSs consist of a repeating mono- or oligosaccharide subunits connected by varying glycosidic linkages, thereby generating homo- or heteropolymers, respectively that are structurally very diverse. They could be covalently linked to bacterial surface molecules forming a capsule, form loosely attached structures, or they can be secreted into the environment. Although very little is known about the structure and biological functions of lactobacilli PSs, it has been suggested that these antigens play an essential role in the adhesion phenomenon. It has been shown that PSs can modify the adhesion of pathogenic bacteria to the intestinal mucus and are considered to be critical in host-microbe interactions, function in immunomodulation.

\section{Natural medium-chain anionic polysaccharides: a significant part of the detoxification phase III system}

\section{Bondarenko I}

North West Russian Research Centre for Hygiene and Public Health, St.Petersburg, Russia, E-mail: igor.g.bondarenko@gmail.com

Humans are exposed to a permanently increasing number of toxic compounds in the environment, including a vast variety of pharmaceuticals. Many of those are very distant from previously encountered compounds, and yet we can cope with environmental exposure by decreasing the level of their toxicity to the body (detoxification). In addition, the excess of endogenous biologically active substances (hormones and neurotransmitters) are recognised as toxic and therefore detoxified via the same metabolic pathways. Considering these exposures, the body's ability to detoxify is now recognised as a key factor to general health. Obvious causative links have been detected between the insufficiency of detoxification and chronic diseases, such as multiple chemical sensitivity syndrome, chronic fatigue, Parkinson's disease, fibromyalgia, allergies, arthritis, certain malignancies, and many more [1]. Most tissues possess detoxifying systems responsible for the transformation and removal of (potentially) toxic chemicals. Proteins that include phase I, phase II enzymes, and phase III transporters play key roles in the detoxification, including elimination, of exogenous chemicals as well as excessive endogenous compounds [2]. Phase II enzymes, such as glutathione S-transferases, sulphotranferases, UDP-glucuronosyl transferases, $\mathrm{N}$-acetyltransferases, and $\mathrm{NAD}(\mathrm{P}) \mathrm{H}$ : quinone oxidoreductases catalyse conjugation of both exogenous and endogenous chemicals. That usually occurs after the reactions of phase I, namely, oxidation, reduction and hydrolysis [3]. Phase III detoxification system, or the antiporter, is an energy-dependent efflux pump that moves xenobiotics from the inside to the outside of the cells. [4]. This is thought to be a mechanism allowing reabsorption, and therefore re-exposure of the non-metabolised xenobiotics to Phase I activity, thus increasing the efficiency of detoxification. Phase III transporters (about 50 in total) are expressed in many tissues, including the liver, intestine, kidney and brain. A substantial role in the excretion of (mostly inorganic) toxins belongs to pectins - carbohydrate polymers consisting mostly of linear chains of polygalacturonic acid. Minor oligosaccharides (like rhamnose, mannose, fructose, and others) form cross-links holding the long chains together. Most pectins can assist in natural oral detoxification because they, being polyanionic chelators, can bind toxic chemicals (mostly metals) while travel along the intestine. Besides that, short- and medium-chain fragments of polygalacturonic acid are able to absorb from the intestine into the bloodstream and bind toxic metals there, with subsequent elimination of the oligosaccharide-bound toxicant into the urine. Moreover, shorter pectin molecule fragments can also stimulate excretion of the inorganic toxicants (lead, mercury, 
manganese, aluminium and others) from inside of the cells [5], which is followed by their urinary excretion. Possible mechanisms of action of different medium-chain anionic polysaccharides (fragmented pectins) as specific components of the detoxification phase III system are discussed.

\section{Dietary therapy for inflammatory bowel diseases}

\section{Dieleman $L^{*}$}

University of Alberta, Edmonton, Canada,E-mail: l.dieleman@ ualberta.ca

The last decade has seen a huge interest in the human microbioma. This was stimulated by the exponential increase of molecular techniques for in depth analysis of even uncultivable micro-organisms. This development was preceded by an increased interest in the gut microflora and its role for the pathogenesis of chronic intestinal inflammation. Several rodent colitis models have indicated that specific gut bacteria induce and perpetuate chronic inflammation. It was also recognized that intestinal inflammation was associated with dysbiosis, with a preponderance of pathogenic-disease inducing bacteria versus a relative lack of beneficial microbes. Such a dysbiosis is not unique to gut-related chronic inflammation, but is also associated with e.g. rheumatoid arthritis, asthma, food allergies, obesity, diabetes and even multiple sclerosis. These findings led to a renewed interest in microflora-altering therapies for these disorders.

One of those chronic inflammatory conditions is human Inflammatory Bowel Diseases (IBD), compromised of Ulcerative Colitis (UC) and Crohn's disease (CD). UC is confined to the colon, whereas Crohn's disease can affect the entire GI tract. IBD is now believed to be caused by a combination of genetic, environmental and immunological factors. Although over 160 susceptibility genes have been identified it requires a combination of such genetic susceptibility and environmental factors to get disease. The high prevalence of IBD in societies with a western lifestyle and the ability to acquire the disease in the next generation by migrating from a low endemic to a high endemic area suggests that environmental instigators are necessary.

Diets can change the intestinal microflora as dietary components form the substrate of these micro-organisms. Recently it was discovered that dietary pattern and lifestyle affect specific bacterial enterotypes. Western diets and especially early exposure to specific gut microflora affect the unique composition of the gut microbioma of that individual. Although the gut microflora is relatively stable after the age of 2-3 years modulations in composition and bacterial functions are possible, recently leading to studies in dietary therapies to prevent and treat mild IBD among others. Although Metchnikov described probiotics already in 1911 it was not until 20 years ago that the role of beneficial microbes was thoroughly investigated in health and disease. Despite enthousiasm for probiotics in IBD only a few well controlled studies have been performed. Only the probiotic cocktail VSL\#3 and E. coli Nissle 1917 were able to prevent and treat mild to moderately active UC and chronic pouchitis, the latter condition after the creation of an ileoanal pouch after colectomy for refractory UC, whereas no effect of probiotics has been shown so far for Crohn's disease. Prebiotics are undigestible dietary fibres that can stimulate specific beneficial microbes to produce short-chain fatty acids in the colon by fermentation. Beta-fructans oligosaccharides can reduce colitis in experimental colitis. Interestingly, in a translational open label study the same prebiotic mixture was able to treat mild to moderately active UC. These beneficial effects were accompanied by specific microflora changes and were associated with increased butyrate metabolism in the responders. Taken together, our increasing knowledge of the gut microbioma and the ability to modulate it has led to an exponential interest in performing studies to prevent and possibly treat chronic diseases that are affected by it. This has led to a renewed interest in assessing the efficacy and potential protective mechanisms of new probiotics, prebiotics, fecal transplantion and dietary therapy as an alternative or adjunct therapy to current anti-inflammatory drugs for chronic debilitating disorders such as IBD.

\section{Gnotobiology as a research tool}

Stepankova $\mathrm{R}^{1^{*}}$, Hrncir $\mathrm{T}^{1}$, Kofronova $\mathrm{O}^{2}$, Grimova $\mathrm{I}^{1}$, Drabonova $\mathrm{B}^{1}$ and Tlaskalova-Hogenova $\mathbf{H}^{1}$

${ }^{1}$ Laboratory of Gnotobiology, Department of Immunology, Institute of Microbiology, Czech Republic

${ }^{2}$ Laboratory Electron Microscopy, Institute of Microbiology, Czech Academy of Sciences, Prague, Czech Republic, E-mail: stepankova. renata@seznam.cz

Gnotobiology is the science of the study of animals or other organisms raised in environments free of germs or those which contain only specifically known germs. Gnotobiotic animals are sterile or associated with well-defined microbiota whereas conventional animals are colonized with unknown germs including bacteria, viruses and parasites. Using the tool of gnotobiotic animals we can determine more precisely how specific germs affect the body.

Our laboratory deals with construction of isolators and obtaining germ-free animals by cesarean section or hysterectomy.

We have established new colonies of germ-free rats and mice which were bred in germ-free conditions. To establish first generation of germ-free colonies the animals were hand-fed with a special formula milk diet designed in our laboratory. In our research we have focused on the role of dietary saturated and unsaturated fatty acids and also showed that milk diet may cause reproductive complications in germfree animals. The sterility of germ free animals is determined by method of cultivation on the plate and fluorescence in situ hybridization (FISH) by oligonucleotide probes. We studied the role of microbiota in immune system development development (eg. T cell development) and in pathogenesis of various diseases (eg. experimetally induced colitis, ankylosing enthesopathy, atherosclerosis).

\section{Mucosal barrier function and inflammatory diseases}

Tlaskalová-Hogenová $\mathbf{H}^{1^{*}}$, Hornová $\mathrm{M}^{1}$, Coufal $\check{S}^{1}$, Kokešova $A^{1}$, Sánchez $D^{1}$, Hoffmanová $I^{2}$, Zákostelská $Z^{1}$, Klimešová $K^{1}$, Dusilová $A^{1}$, Málková $J^{1}$, Štěpánková $R^{1}$, Hudcovič $T^{1}$, Kozáková $H^{1}$, Rossmann $\mathrm{P}^{1}$ and Kverka $\mathrm{M}^{1}$

${ }^{1}$ Institute of Microbiology, Academy of Sciences of the Czech Republic, v.v.i., Videňská 1083, Prague 4, Czech Republic

${ }^{2} 3 r d$ Faculty of Medicine, Charles University, Prague, Czech Republic, E-mail: tlaskalo@biomed.cas.cz

The steadily increasing prevalence of chronic diseases like allergy, arthritic diseases, inflammatory bowel diseases, diabetes, obesity and others occuring in developed countries is causing significant morbidity. These disorders represent an important medical problem because they have a devastating impact on quality of life and require long-standing medical care. Considerable effort has been made to understand mechanisms leading to the development of these disorders. 
Numerous chronic diseases may occur as a result of disturbances of mucosal barrier function or changes in mechanisms regulating mucosal immunity. Barrier formed by intestinal epithelium separates intestinal microbiota from underlying tissues, preventing bacterial infiltration and subsequent inflammation. The interaction is usually balanced however under some conditions this interaction could be for the host harmfull. The failure of gut barrier function is important feature in pathogenesis of several inflammatory and neoplastic diseases. We analysed how systemic inflammation influences the intestinal barrier function and how indometacin-induced barrier dysfunction differs between germ-free and conventional mice. We demonstrated that systemic inflammation increases the gut barrier permeability and changes the expression of pro inflammatory cytokines and tight junction proteins in the colon mucosa. We found that the presence of commensal microbiota is necessary for indometacin induced gut barrier failure and that probiotics or their lysates can restore intestinal barrier function. Inadequate intestinal colonization with premature delivery, delivery by Cesarean section and excessive use of perinatal antibiotics results in the absence of adequate bacterial-epithelial crosstalk and an increased incidence of immune-mediated diseases (e.g. necrotizing enterocolitis - NEC, allergy). Necrotizing enterocolitis, an intestinal inflammatory disease of premature infants, is caused, in part by an excessive inflammatory response to initial bacterial colonization due to the immaturity of innate immune system. We studied the possibility to use biomarkers of intestinal mucosal cell damage as diagnostic tool for distinguishing early NEC from sepsis. Our results suggest that intestinal fatty acid binding protein (i-FABP) can distinguish early stadium of NEC from sepsis. Infants with inadequate intestinal colonization can be restored to a bacterial balance with the intake of probiotics.

\section{Application of high hydrostatic pressure to increase microbiological stability of unfiltered beer}

Dostálek $\mathrm{P}^{{ }^{*}}$, Kotlíková $\mathrm{B}^{1}$, Tůmová $\mathrm{T}^{1}$, Ivanišová $\mathrm{M}^{1}$, Strohalm $\mathrm{J}^{2}$, Houška $M^{2}$, Jelínek $L^{1}$ and Karabín $M^{1}$

${ }^{1}$ Department of Biotechnology, Institute of Chemical Technology Prague, Technická 5, 16628 Prague 6, Czech Republic

${ }^{2}$ Department of Food Engineering, Food Research Institute Prague, Rádiová 7, Prague 10, Czech Republic, E-mail:Pavel.Dostalek@vscht.cz

The shelf-life of unfiltered beer is mostly affected by living yeast and, as such, can be increased by pasteurization. Using this method, increased microbiological stability can be achieved, but heating beer during pasteurization has a negative effect on its sensory stability. The application of high hydrostatic pressure (HHP) to unfiltered beer, instead of pasteurization, should inactivate the yeast, thus avoiding this problem. Samples of non-filtered beer were subjected to HHP from 250 to $450 \mathrm{MPa}$ for 5 or 10 minutes and then tested for microbial viability. The results show that HHP (350 MPa for 5 minutes) apparently inactivated yeast cells very effectively, and we did not observe any lysis or leakage of intracellular components into the beer. Samples tested for microbial activity showed no cell growth on nutrient agar, however, using flow cytometry and a dead yeast cell staining method, we showed that around $70 \%$ rel. of cells were still viable. Subjecting samples to $350 \mathrm{MPa}$ HHP for 10 minutes instead of 5 had almost the same effect on yeast cell viability. By applying higher pressures (450 MPa), up to $85 \%$ rel. of cells were killed, but this led to undesirable leakage of intracellular components; thus a lower pressure is recommended. These results suggest that application of high hydrostatic pressure may be a promising technique for increasing the shelf-life of unfiltered beer.
Manufacturing method for semi-dried mulberry fruit having improved stability of cyanidin-3-glucoside and preserved shape

\section{Kim $H^{*}$, Kim S, Jo Y, Kweon H and Lee K}

National Academy of Agricultural Science, Korea, E-mail: hyunbok@korea.kr

As mulberry fruit's functionality has known through the media and public relations, attention on consumption and processing products has been increased in Republic of Korea. However, mulberry fruit is common to keep a frozen storage because of the very high water content. Because freezing-storing cost generates before the processing or sales, farmers of mulberry fruit production are weighted in financial burden. In addition, mulberry fruit has difficulty in expanding consumption by made in the simple processed products like the mulberry jam, mulberry juice, mulberry enzyme, alcohol, etc. Recent, frozen fruits including frozen blueberries, frozen strawberries, frozen mango, frozen rich, frozen mulberry fruit has been started to sell in the large discount stores and convenience stores.

However, there is restricted sales in only special place that have the frozen distribution facilities. So, for the income improvement and consumption promotion of mulberry farmers facing these difficult situations, we developed the method of semi-dried mulberry fruit using the hot air dryer.

By this method, it was possible to manufacture three kinds of processing products (juice, jam, semi-dried mulberry fruit) at the same time. First, to make the mulberry juice, put the sugar into the mulberry fruit in a ratio of $1: 1$, and citric acid $0.3 \%$. It was fermented at room temperature for 2-4 weeks. After the filtering the its juice, mulberry jam was manufactured just grinding fermented mulberry fruit. The rest fermented mulberry fruit was inserted in the hot air dryer $(35 \sim 40 \rrbracket)$ for 24 30hours. This semi-dried mulberry fruit maintained the shape and cyanidin-3-glucoside stability compared to other treatment group. Also, it can be preserved at room temperature.

Phytochemicals in berberis vulgaris downregulate virulence gene expression in enteropathogenic $E$. coli by quorumquenching and block intimin-mediated attachment on human enterocytes

Oyong $\mathrm{G}^{1,2^{*}}$, Jafari $\mathrm{N}^{3,4}$, Tan $\mathrm{MC}^{5}$ and Cabrera $\mathrm{E}^{2}$

${ }^{1}$ Molecular Science Unit, Center for Natural Science and Ecological Research, De La Salle University, Manila, Philippines

${ }^{2}$ Biology Department, De La Salle University, Manila, Philippines

${ }^{3}$ Department of Cell and Molecular Biology, College of Science, University of Tehran, Tehran, Iran

${ }^{4}$ Markey Cancer Center, University of Kentucky, USA

${ }^{5}$ Chemistry Department, De La Salle University, Manila, Philippines, E-mail: glenn.oyong@dlsu.edu.ph

Enteropathogenic Escherichia coli (EPEC) is a significant cause of diarrhea among the young and the elderly worldwide. This coliform pathogen intimately attaches on human enterocytes via expressed virulence factors induced by quorum-sensing (QS) mechanism. The QS inhibition (QSI) properties of several phytochemicals from Berberis vulgaris L. var. asperma fruit ethanolic extract (BFE) against EPEC O55:H7 pathogenesis and virulence were investigated. Transcription 
profiles of virulence factors eaeAg (intimin), escC (type III secretion biogenesis), and tir (translocated intimin receptor) were respectively quantified by RT-qPCR post-exposure to B. vulgaris ethanolic extract. Moreover, co-treatment of EPEC planktonic cultures with BFE seeded and exposed on human enterocytes (FHC) was performed in vitro to determine bacterial attachment and colonization. FHC and EPEC were correspondingly stained with antibody-tagged probes and visualized by fluorescence microscopy. In silico analyses of molecular interaction of phytochemicals, previously identified by LC-MS, on the TIR binding site was assessed via LigandFit. A significant down-regulation in the expression of eaeA, escC, and tir $(\mathrm{p}<0.05)$ was observed from as low as $20 \%$ of BFE. Fluorescence photomicrography revealed inhibition of localized adherence as opposed to untreated controls. Identified phytochemicals chlorogenate and rutin showed high dock scores dictated by their molecular interaction with TIR. Results infer the QSI potential of $\mathrm{B}$. vulgaris against EPEC as evidenced by non-adherence on FHC. These findings suggest prevention of infection, colonization and virulence of EPEC via quorum-quenching and blockade of TIR by phytochemicals in the $\mathrm{B}$. vulgaris fruit ethanolic extract.

Monolithic and core-shell columns in fast uni-, two-and three-dimensional hplc separations of phenolic compounds

\section{Jandera $P^{\star}$, Hájek $T$ and Staňková $M$}

University of Pardubice, Department of Analytical Chemistry, Studentská 595, 53210 Pardubice, Czech Republic, E-mail: Pavel. Jandera@upce.cz

UHPLC on sub-2 micron particle columns is a straightforward way to increasing the speed of HPLC separations, at the cost of very high pressures and special instrumentation. However, fast efficient separations are possible also on short core-shell or monolithic columns and often enable fast (1 min or less) and efficient separations at pressures which can be achieved with conventional HPLC instrumentation, due to low flow resistance. This feature is especially attractive for fast separations in the second dimension of comprehensive twodimensional HPLC, where the analysis of a fraction collected from the first dimension must be performed on-line in the second dimension within the short time available for the collection of the next fraction. Short core-shell or silica-based monolithic columns in the second dimension can be combined with a longer microbore or capillary column in the first dimension. For this purpose, we developed an efficient organic polymer zwitterionic column, which can be used either in reversed-phase or in normal-phase (HILIC) mode for efficient separations of phenolic acids, polyphenolic compounds, flavonoids and related compounds. Selection of suitable column combinations and separation conditions are discussed. Dramatic increase in the number of compounds separated in a single run can be achieved by applying simultaneous gradient elution on a zwitterionic monolithic microcolumn in the first dimension and on a $3-5 \mathrm{~cm}$ core-shell or silicabased monolithic column in the second dimension. As the monolithic zwitterionic polymethacrylate column shows dual retention mechanism in mobile phases with low and high concentrations of acetonitrile, alternating RPxHILIC and RPxRP separations with increasing and decreasing acetonitrile gradients run serially in the first dimension can be performed with the same column combinations, in a quasi-threedimensional system for further increase of the peak capacity up to over 900. The approach is illustrated by examples of separations of complex standard mixtures of phenolic, polyphenolic and flavonoid compounds and analysis of beverages and plant extracts.

\section{Novel analytical approaches to on-site analysis applicable to nutrition and food analysis}

\section{Macka $\mathbf{M}^{*}$}

Australian Centre for Research on Separation Science (ACROSS) and School of Physical Sciences - Chemistry, University of Tasmania, Private Bag 75, Hobart 7001, Australia, E-mail: mirek.macka@utas.edu. au

The need for portable analysis to undertake on-site or in-field analysis of complex mixtures has been increasing over the past decades. The progress in miniaturisation of instrument components and the development of ultra-small controllers and data processers has opened up analytical techniques traditionally seen as bound to the lab bench, such as separation methods, to portability and thus on-site use. At the same time new concepts have been developed allowing low-cost analytical approaches, such as based on paperfluidics. In this presentation the increasingly effective applicability of analytical methods for portable on-site analysis will be illustrated using two examples: (1) Miniaturised capillary liquid chromatography (LC), and (2) Novel paperfluidics based analytical devices ( $\mu$ PADs), with attention paid to their applicability in the areas of nutrition and food analysis.

In our approach to miniaturised capillary LC, our goal was to design a portable medium-pressure highly flexible modular 'LEGOstyle' LC system based almost entirely from commercial off-the-shelf low-cost components. The system weighing under $2 \mathrm{~kg}$ (plus data acquisition) is based on a breadboard assembled modular flexible microfluidic system (LabSmith) with microsyringe pumps (5, 20 and $100 \mu \mathrm{L}$ ), complemented with other off-the-shelf components, including an injection valve and LED-based photometric detector, all operated through a PC.

Paper-based microfluidic devices ( $\mu$ PADs) are capable of achieving rapid qualitative and quantitative measurements of a variety of analytes inexpensively. We developed a novel, highly flexible and low-cost fabrication method using a desktop digital craft plotter/cutter and technical drawing pens with tip size of 0.5 and $1.0 \mathrm{~mm}$. The pens were used with either commercial black permanent ink for drawing fluidic brakes, or with specialty in-house formulated aqueous inks. A functional test of these $\mu$ PADs was conducted by the screening of antioxidant activity. Photometric measurements of flavonoid, phenolic compounds and DPPH free radical scavenging activity were carried out on $\mu$ PADs.

\section{LC/EC/MS for the prediction of metabolism and toxicity of drugs: what shall $i$ do if $i$ have got flu?}

\section{Horna $A^{\star}$ and Vyňuchalová $K$}

Institute of Nutrition and Diagnostics, RADANAL Ltd., Okružní 613, Pardubice, Czech Republic, E-mail: horna@radanal.cz

Paracetamol (Acetaminophen, $\mathrm{N}$-acetyl-p-aminophenol) is an effective analgesic-antipyretic that can be administered safely to children and adults at the appropriate therapeutic doses. The drug is readily metabolised by the liver and toxic metabolites are removed by conjugation with endogenous glutathione. Accidental or deliberate overdosage can lead to hepatic toxicity that can be fatal if not treated urgently with antidotes that replenish depleted glutathione. Pharmacists have an important role to play in patient education to help reduce the incidence of paracetamol poisoning. At the therapeutic 
adult dose of 1-2 g/day, oral paracetamol is indicated for fever and mild to moderate acute pain conditions. Administration of acetaminophen via intravenous route has become increasingly widespread and has been used as a safe and effective antipyretic and analgesic agent. Maximum recommended therapeutic dose of paracetamol is $4 \mathrm{~g} /$ day in adults and $50-75 \mathrm{mg} / \mathrm{kg} /$ day in children. Consumption of a single dose greater than $7 \mathrm{~g}$ in an adult and $150 \mathrm{mg} / \mathrm{kg}$ in a child is considered potentially toxic to the liver and kidneys due to the highly active metabolite $\mathrm{N}$-acetyl-p-benzoquinone imine. Paracetamol is essentially metabolized in the liver by conjugation with glucuronic acid (55\%) and sulfuric acid (35\%). Hepatotoxic metabolites are produced in small amounts by the cytochrome P450 (isoenzyme CYP2E1). In the therapeutic plasma concentration range, this metabolite is detoxified by conjugation with glutathione. In case of intoxication the amount of this toxic metabolite increases and outweighs the amount of available glutathion, which can lead to hepatic failure and renal tubular necrosis. Paracetamol metabolism is age- and dose-dependent. Metabolites are excreted through the kidneys in the urine. Only $2-5 \%$ of the dose is excreted in an unchanged form in the urine. As a consequence of its short elimination half-life (1-3h), 24 hours after the ingestion of a single dose of paracetamol, $98 \%$ of the dose is eliminated. A prediction of toxicity and metabolism in a human body is very important for determining of harmfulness of compounds which affect us or substances that we intentionally accept thinking that they aren't toxic. A suitable tool for mimicking the drugs metabolism in a human body is liquid chromatography coupled with mass spectrometry and electrochemistry (LC/EC/MS). Usage of electrochemistry allows simulating of the metabolism catalysed by cytochromes P450. Gained oxidation products are analysed and identified with mass spectrometry. Moreover it is possible to watch interactions between metabolites and glutathione thanks to injecting of this tripeptide directly to the device.

Sample pretreatment for analysis of benzodiazepines in serum and urine

\section{Mucha M1,2*, Kurka P3 and Kalina J1,2}

${ }^{1}$ Department of Chemistry, Faculty of Science, University of Ostrava, 30. dubna 22, 70133 Ostrava, Czech Republic

${ }^{2}$ Environmental Center, Faculty of Science, University of Ostrava, 30. dubna 22, 70103 Ostrava, Czech Republic

${ }^{3}$ Department of Forensic Medicine, University Hospital Ostrava, 17. listopadu 1790, 70852 Ostrava - Poruba, Czech Republic, E-mail: marek.mucha@osu.cz

Benzodiazepines are currently the most popular psychotropic drugs among physicians and drug addicts. Their ever increasing consumption and hence necessity of their accurate determination is associated with this popularity. The determination of benzodiazepines requires powerful analytical instrumental methods as for example LC/ MS. However, it is unsafe to make a direct injection of real sample due to risk of instrument contamination. Therefore, sample pretreatment by for example SPE or Liquid-Liquid (L-L) extraction is normally used before instrumental analysis $[1,2,3,4]$.

The present work describes optimization of L-L extraction procedure for pretreatment of real serum and urine samples containing six selected benzodiazepines (Alprazolam, Bromazepam, Clonazepam, Diazepam, Chlordiazepoxide and Oxazepam) for analysis by LC/MS. Different types and volumes of extraction agents and buffers were tested and method parameters were optimized. The extracts were analyzed by LC/MS system.
The optimized L-L extraction method with recovery $67-88 \%$ for serum and $65-92 \%$ for urine was tested in a wide concentration range of bezodiazepines and it was compared with SPE. This extraction method was subsequently applied for determination of selected benzodiazepines in real samples of serum and urine.

The ods vitamin D initiative: information, standardization, and analytical tools for assuring high quality clinical measurements of vitamin d metabolites

\section{Betz ${ }^{1 \star}$, Sempos $C^{1}$, Wise $S^{2}$ and Coates ${ }^{P}$ \\ ${ }^{1}$ NIH Office of Dietary Supplements, USA \\ ${ }^{2}$ National Institute of Standards and Technology, USA, E-mail: betz@od.nih.gov}

A landmark paper published in 2004 (1) highlighted the diversity of analytical approaches to clinical Vitamin D status determination and the variability of analytical results resulting from the various analytical platforms. The paper called for the creation of a Vitamin D laboratory standardization program and identified a series of resources that would be required in order to initiate the program. The Office of Dietary Supplements (ODS) at the U.S. National Institutes of Health partnered with the U.S. National Institute of Standards and Technology (NIST) to develop a Vitamin D Initiative (2) with analytical and informational resources and to begin the process of organizing an international Vitamin D Standardization Program (VDSP) (3). The ODS/NIST collaboration produced certified reference materials (CRM) for 25-hydroxyvitamin D in human serum (SRM 972a) and 25-hydroxyvitamin D calibration solutions (SRM 2972a). Additional clinical CRM for higher levels of Vitamin D metabolites in serum (SRM 2973) and in calibration solutions are in progress. Creation and dissemination of the CRM permitted development of a Reference Measurement Procedure (RMP) for vitamin D metabolites in human serum. The goal of the VDSP is to promote standardized laboratory measurement of 25-hydroxyvitamin D to improve clinical results and public health. Standardization permits all participating laboratories to report true values based on NIST and University of Ghent RMPs. True values permit pooling of research results, which in turn enables creation of evidence-based guidelines and more informed decision making by physicians, policymakers, and others.

\section{Community based alternative management of fibroids using nutritional and herbal supplements among women in kisumu municipality}

\section{Aruwa $\mathrm{S}^{1 \star}$, Otieno $\mathrm{A}^{2}$ and Rew $\mathrm{F}^{3}$}

${ }^{1}$ Agriculture Sector Development Strategy Programme, Siaya County, Kenya

\section{${ }^{2}$ Uzima University, Kenya}

33aramogi Oginga Odinga University, Kenya, E-mail: suaruwaus@ yahoo.com

Background: Fibroids are among the most common conditions affecting women of reproductive age, 70 to $80 \%$ of women will have had one by the age of fifty. Introduction:In Kenya $80 \%$ of the populations are still dependent on herbal medicine. The World Health Organization's global strategy includes popularizing and incorporating herbal medicine in the national health systems of member countries. Worldwide, people developed unique indigenous healing traditions adapted and defined by their culture, beliefs and environment. In 
the African context many plants have been used in traditional herbal medicines in an attempt to treat the uterine fibroids. Many of these have slowly reduced the size of fibroids and prevent further growth of new ones.

Methodology: The study used quantitative and qualitative methods of data collection, the study population were women diagnosed with fibroids who had attended alternative medical care. The sample size was 347 .

Conclusions: The study established that fibroids can be completely eliminated using nutritional and herbal supplements. Of the 350 women interviewed $9.4 \%$ had fibroids completely cleared. Those who used nutritional supplements were at $6.2 \%$, while those who used indigenous processed herbs were $3.1 \%$. Fear of surgery, its complications and lack of hope for complete removal of fibroids were the main reasons women turned to using nutritional and herbal supplements.

\section{Quality considerations in chondroitin sulfate products Hildreth $\mathbf{J}^{*}$ \\ Synutra Pure, Ltd., USA, E-mail: jhildreth@synutrapure.com}

Chondroitin sulfate (CS) is a major component of connective tissue, and is partially responsible for providing the flexibility of these tissues. A recent systematic review on "Chondroitin Sulfate for Osteoarthritis" reviewed 43 clinical trials conducted over more than 20 years and involving 9,110 participants who used chondroitin for osteoarthritis (OA). The review concluded that chondroitin performed better than placebo by safely improving pain and functional capacity in OA patients. Chondroitin sulfate is marketed in a variety of quality levels and concentrations and in many countries there is no required standard dosage. In the U.S. market, CS is sold as a dietary supplement and supplement product manufacturers are not permitted to make any claim related to treatment of osteoarthritis. The United States Pharmacopeial Convention (USP) publishes a chondroitin monograph in its Dietary Supplement Compendium that mandates a suite of testing methods to ensure purity and quality of this ingredient and an assay result of better than 90 percent CS. In European markets, chondroitin is sold both as an approved slow-acting drug for symptomatic relief of osteoarthritis (SYSADOA) as well as a nutritional supplement. Both the European Pharmacopeia and the British Pharmacopeia define pharmaceutical grade chondroitin as $95 \%$ or better in assay content. Chondroitin material that does not meet this assay requirement is not considered to be in compliance with drug quality standards, although nutritional supplements that contain less than 95\% CS are available. When considering evidence for efficacy of CS in osteoarthritis, it is important to note that most of the existing clinical evidence for CS efficacy derives from clinical trials that used pharmaceutical grade chondroitin sulfate as the active intervention. A recent study of the quality of CS products marketed in the Czech Republic as prescription drugs and nutraceutical found that the majority of products sold as Nutraceuticals did not meet their label claim CS but that products sold as drugs met claims, probably because of mandatory drug manufacturing regulations. Chondroitin sulfate has been proven to be a useful ingredient for treating the symptoms of osteoarthritis but variability in the regulatory environment where it is sold and in the quality of raw materials and finished products 2 is confusing to consumers. This talk will provide an overview of the quality issues and the scientific challenges surrounding CS quality and testing.

\section{Biomarkers of (anti)oxidant status in human nutrition, aging and disease}

\section{Jansen $\mathrm{E}^{1 \star}$, Beekhof $\mathrm{P}^{1}$, Cremers $\mathrm{H}^{1}$ and Ruskovska $\mathrm{T}^{2}$}

${ }^{1}$ Centre for Health Protection, National Institute for Public Health and the Environment, Bilthoven, the Netherlands

${ }^{2}$ Faculty of Medical Sciences, Goce Delcev University, Stip, Macedonia, E-mail: eugene.jansen@rivm.nl

The (anti)oxidant status of individuals is an important factor for the risks of chronic diseases. Biomarker measurements in serum/plasma is a good way to determine the status of the oxidant/antioxidant balance. From our own experience, we come to a proposal of a set of biomarkers for nutritional intake of antioxidants to determine the (anti)oxidant status in serum as a reflection of nutrition. Serum concentrations of biomarkers of fat-soluble vitamins are not suitable to assess transient changes in nutritional intake, because of their strong homeostasis. For long-term epidemiological studies however, they are well-suited. In addition, a number of oxidation/redox biomarkers can be used in addition to the antioxidant to assess possible relations with health risks. The best biomarkers for this purpose are the reactive oxygen metabolites and total thiols. Examples will be given from large-scale European studies. With the combination of vitamin A, vitamin E, carotenoids (as measured with HPLC), vitamin C, reactive oxygen metabolites, biological antioxidant potency and total thiols (as measured with an auto-analyzer), an overall view is obtained concerning the intake and effect of antioxidants in nutrition and aging research. In this paper we describe the methods to measure a selection of these biomarkers in general population. Critical points in biomarker validation with respect to blood sampling, storage conditions and assay stability are discussed.

\section{A genetic immunonutrition research experience}

\section{Mabrouk RR*}

Faculty of Medicine, Ain Shams University, Cairo, Egypt, E-mail: randareda@gmail.com

The interrelation of the immune system, genetic variants, and the environmental factors including the effective role of nutrients on the body system in health and disease has always been interesting. Lately it was the focus of research work. Most common disorders that have a genetic component such as obesity, diabetes, heart diseases, common forms of cancer, most autoimmune diseases and allergic diseases, have both a genetic and environmental component. Though genetics sets the predisposing background for these disorders, yet prevention depends largely on the response of the immune system to the environmental factors. Nutrigenetics studies the role of genetic variation on interactions between diet and health, aiming to provide more personalized dietary advice. Many serious disorders and morbidities can be prevented if the nutritional needs are tailored according to the genetic profile. To our knowledge, no researchers in Egypt had studied the relation between the genes and the nutrients in the different disorders. In our newly developed immunutrition research unit in the Immunology, Clinical Pathology Department at the Faculty of Medicine, Ain Shams University Cairo Egypt an ongoing research performed by the unit young researchers together with the academic faculty on the genetic based disorders including Hypertension, Obesity, \& Metabolic Syndrome has started. We aimed to open up some important new information in relation to dietary optimization. Genetic variants analysis using the PCR and the single nucleotide polymorphism in the above mentioned disorders is being investigated. 
We are analyzing the TCF7L2 in Type II DM, the NOS3 and ApoA2 gene in familial hypercholestraemia and in co-morbitidities related to obesity, and the ACE gene variants in hypertensives. The results are correlated to the severity of the diseases, immunological parameters related to both the innate and adaptive immune system together with different nutrients concentration. The results related to the dietary habits and immunological are very interesting and informative.

Impact of elevated levels of plasma homocysteine and impaired dna repair in a mouse model of vascular cognitive impairment

Jadavji NM ${ }^{1,2^{*}}$, Füchtemeier $M^{1}$, Boehm-Sturm $P^{1}$, Farr TD $^{1}$, Smith $\mathrm{PD}^{2}$, Malysheva $\mathrm{O}^{3}$, Caudill $\mathrm{MA}^{3}$ and Dirnagl $\mathrm{U}^{1}$

${ }^{1}$ Department of Experimental Neurology; Center for Stroke Research Berlin, Charité Medical University, Berlin; German Center for Neurodegenerative Diseases, Partner Site Berlin, Germany

${ }^{2}$ Carleton University, Canada

${ }^{3}$ Division of Nutritional Sciences, Cornell University, Ithaca, USA, E-mail:nafisa.jadavji@mail.mcgill.ca

Epidemiological evidence indicates that folate deficiency plays a role in the development of vascular cognitive impairment (VCI). Folates are involved in nucleotide synthesis, including DNA repair and methylation. Uracil-DNA glycosylase (UNG) plays a role in DNA repair and previous work has shown that folate deficiency in UNG deficient mice (UNG-/-) results in neurodegeneration. Deficiencies in folate metabolism result in increased levels of homocysteine. Methylenetetrahydrofolate reductase (MTHFR) is an enzyme that links folate and homocysteine metabolism. Mthfr+/- mice have elevated levels of plasma homocysteine, but are otherwise phenotypically normal. The purpose of this study is to evaluate the impact of genetic deficiency in folate metabolism or impaired DNA repair in combination with nutritionally induced hyperhomocysteinemia in a mouse model of VCI produced by chronic hypoperfusion. Male and female mice deficient in MTHFR (Mthfr+/-) or UNG (UNG-/-) and wild-type controls were placed on either control (CD) or folate deficient (FADD) diets for 6 weeks after which mice underwent implantation of microcoils around both common carotid arteries or a sham procedure. Behavioural and magnetic resonance angiography analysis began after 4-weeks. At the conclusion of experiments plasma homocysteine levels were measured and choline metabolism was assessed in cortical tissue. All mice on FADD had elevated levels of plasma homocysteine. Angiography showed remodeling of the basilar artery and vasculature at the base of the brain in wildtype CD and FADD microcoil mice. FADD UNG/- microcoil mice exhibited learning impairments in the Morris water maze whereas MTHFR-deficient mice did not. Only MTHFR mice with chronic hypoperfusion had altered choline metabolism in cortical tissue. The results suggest that a combination of hyperhomocysteinemia, chronic hypoperfusion or impaired DNA synthesis is required for memory impairments, whereas MTHFR deficiency alone has no effect on learning. Additionally, cortical choline metabolism is disrupted in MTHFR deficient mice with chronic hypoperfusion.

\section{Cancer screening using tumour markers}

\section{Buchler $\mathbf{T}^{\star}$}

Department of Oncology, First Faculty of Medicine and Thomayer Hospital, Charles University, Prague, Czech Republic, E-mail: tomas. buchler@ftn.cz
Tumour markers are chemical compounds produced by tumour tissue. They are either completely absent or present in significantly lower concentrations in a healthy individual than in the presence of a tumour. Circulating tumour markers are mostly proteins and glycoproteins, although some microRNA molecules have been characterised as tumour markers in the recent years. The concentration of tumour markers in the plasma or other body fluids depends on the tumour size, degree of secretion of the tumour marker by cancer cells, and metabolic degradation of the tumour marker molecules. Although the importance of tumour markers in the clinical practice is often overrated by both physicians and patients, they have become an important part of the diagnostic process for many malignancies. However, the detection of early cancers using tumour markers has remained an elusive strategy despite significant efforts and many clinical trials, especially in prostate cancer using prostate-specific antigen (PSA) and in ovarian cancer using carbohydrate antigen 125 (CA 125).

Prostate specific antigen (PSA). PSA is a prostate-specific serine protease. PSA monitoring is of paramount importance for early prostate cancer detection and for monitoring of patients during and after treatment. Benign causes of PSA elevation include invasive medical procedures (urinary bladded catheterisation, cystoscopy, prostate biopsy), inflammation (prostatitis, prostatic abscess), as well as ejaculation (where PSA normalises after approximately 48 hours). Some drugs reduce PSA concentration - for example finasteride, an inhibitor of 5-reductase, lowers serum PSA levels by an average of 50\%. Nevertheless, PSA has quite high sensitivity and specificity for prostate cancer and correlates with prostate cancer clinical stage, with values above $20 \mathrm{ng} / \mathrm{ml}$ associated with an $80 \%$ risk of extraprostatic tumour progression. PSA is frequently used for the screening of healthy men for early prostate cancer. However, the strategy has generated significant controversy as most population-based studies failed to confirm the benefit of PSA screening in terms of overall mortality. Consequently, PSA screening has not been adopted by most health systems despite the fact that prostate cancer is the most common type of cancer of men in the developed world. Carbohydrate antigen 125 (CA125). CA 125 is a differentiation antigen expressed during the development of pleural, peritoneal, and scrotal serous membranes. CA125 levels are increased in serous ovarian carcinoma (sensitivity 82-96\%), one of the most common and deadly gynaecological cancers. Ovarian cancer is notoriously difficult to detect in the early stages by clinical or radiographic examination. However, CA125 has relatively low specificity and may be elevated in any disease affecting the serosal surfaces, such as endometriosis, effusion or inflammation. Alone or in connection with ultrasonographic examination, CA125 has been used for the screening of ovarian cancer, although the benefits of this approach in terms of prolonging the life of patients have not been unequivocally demonstrated.

\section{The role of nutrition in preventive oncology}

\section{Mach I}

\section{Czech Association of Nutritionists, E-mail: ivan.mach@nutris.net}

It's been shown that nutrition and the parameters of its framework are important, if not the dominant risk factor for a variety of malignant tumors. So far, the influence of nutrition was only not proven on the breast cancer. For this reason the nutritional advice offered by the clinical oncologic workplaces to the potential cancer patients and their direct relatives is still missing. In the upcoming period it should be a part of a comprehensive approach to the prevention of malignant neoplasms and their recurrences. It is expected that by the year 2030 
cancer will be the leading cause of death among the Czech population. At the specialized workplaces equipped with the necessary diagnostics, we expect trained experts to be able to create nutritional plans with the aim of promoting the prevention and therapy of oncologic diseases. It is known that a wide range of nutritional substances that bind to known to us carcinogens neutralize these formations, reduce angiogenesis of the tumors, as well as the progression of cancer cells themselves or blocks certain involved enzymes. These chemo-protective and chemopreventive agents that have a mostly antioxidant properties are of either plant or animal origin. In cancer prevention a great advantage can be provided by the use of phytochemicals found in berry plants (anthocyanidines, carotenoids, ellagic acid, resveratrol), onion (diallyl disulfide, quercetin), legumes (phytoestrogenes like genistein as well as other polyphenoles and isoflavones), Brassicae (glukosinolates, isothiocyanates, indoles), citrus (limonen), ginger (Curcumin), Solanaceae (carotenoids, lycopene) and green tea (epigallokatechin gallate). The protective animal factors include vitamin $\mathrm{D}$ and some of the fatty acids. For example, conjugated linoleic acid (lamb, mutton and sheep's milk cheese) and in particular the n-3 unsaturated fatty acids (omega-3) sourced from enriched and purified fish oil supplements are generally anti-inflammatory. Studies on animals have shown the effects of these and many other substances (containing Zn, Se etc. - anticancer enzyme catalyst etc.) to reduce the risk of breast, colon and prostate cancer, and are known to enhance the effectiveness of certain types of chemotherapy. Currently the pharmaceutical industry offers a number of substances in the form of food supplements, manufactured in accordance with the principles of good manufacturing practice (GMP) suitable for preventive supplementation.

Comparison of the polyphenolic profiles of berries from different vaccinium species in relation to their nutraceutical value

Ancillotti C1 ${ }^{33^{*}}$, Ciofi $\mathrm{L}^{1}$, Chiuminatto $\mathrm{U}^{2}$, Stahl-Zeng $\mathrm{J}^{2}$, Bartoletti $\mathrm{R}^{3}$ and Del Bubba $M^{1}$

${ }^{1}$ Department of Chemistry, University of Florence, Via della Lastruccia 3 - 50019 Sesto Fiorentino, Florence, Italy

${ }^{2}$ Sciex Europe, Landwehrstraße 54, 64293 Darmstadt, Germany

${ }^{3}$ Department of Experimental and Clinical Medicine, University of Florence, Largo Brambilla 3 - 50134, Florence, Italy, E-mail: claudia. ancillotti@unifi.it

Vaccinium myrtillus is a wild berry native to mountain areas of Northern and Central Europe, largely used in transformed products, as well as dietary supplements. The interest in this fruits is due to its high content of phenolic compounds, well-known for their healthprotecting attributes, as anti-inflammatory, anti-hypertensive, antimicrobial and anti-cancer agents. Accordingly, many researches focusing on the characterization of selected phenolics in V. myrtillus berries have been published in recent years, evidencing that the most abundant class of polyphenols in V. myrtillus berries are anthocyanins. The composition of phenolic compounds of V. myrtillus berries has been found different from the one of other Vaccinium species - such as the widely commercialized V. corymbosum - indicating the potential use of phenolic profile for the chemotaxonomic discrimination of $\mathrm{V}$. myrtillus fruits from other cultivated and wild species. This aspect is very important since V. myrtillus shows a nutraceutical value higher than that of V. corymbosum and the two species are not well-distinguished by consumers. However, to the best of our knowledge, no comprehensive investigation of the polyphenolic profiles of these Vaccinium species has been published. Moreover, the presence of a different Vaccinium species, namely V. gaultherioides (for which no data regarding the primary and secondary metabolic profiles are reported in literature) has been recently observed in the zones traditionally populated by V. myrtillus, such as Tuscan Apennines. In this regard, it should be underlined that the phenotype of $\mathrm{V}$. gaultherioides berry is very similar to the one of V. myrtillus and the two berries can be confused by the harvesters involved in the production chain of transformed bilberry. For this reason, the polyphenolic profile of V. myrtillus, V. corymbosum and V. gaultherioides berries have been comparatively investigated using liquid chromatography coupled with non-target tandem quadrupole-time of flight mass spectrometric approaches for obtaining comprehensive metabolomic profiles of polyphenolic fractions. The results showed that the three species exhibit very different quali-quantitative profile of phenolic substances, can be discriminated according to their polyphenolic compositions and can be successfully used for health purposes according to their specific characteristics.

\section{Athena study: antioxidants and cardiovascular risk}

Brasacchio $\mathrm{C}^{1,3 \star}$, Conti $\mathrm{C}^{1}$, Soldati $\mathrm{L}^{1}$, Dogliotti $\mathrm{E}^{2}$, Lazzaroni $\mathrm{M}^{3}$, Mingione $\mathrm{A}^{1}$ and Cusi $\mathrm{D}^{1}$

${ }^{1}$ University of Milan, Department of Health Sciences, San Paolo Hospital Milan, Italy

${ }^{2}$ Umberto Veronesi Foundation, Milan, Italy

${ }^{3}$ I.R.C.C.S. Carlo Besta Foundation, Milan, Italy, E-mail: caterina. brasacchio@unimi.it

According to World Health Organization data, the leading causes of death are cardiovascular diseases (CD). Nutritional studies demonstrated a protective role of antioxidants against $\mathrm{CD}$, cancer and obesity. The European study ATHENA aims to study the beneficial effects of antioxidants on human health. We investigated the dietary intake of anthocyanin, vitamin $\mathrm{C}$, vitamin $\mathrm{E}$ and beta-carotene in relation to some of cardiovascular risk parameters (BMI, waist circumference, cholesterol LDL and HDL, LDL/ HDL ratio, triglycerides) in 493 Caucasian subjects divided in two groups: Group 1 (low antioxidants intake) vs. Group 2 (high antioxidants intake). Statistical analysis was performed using STATA 10 Software. The Group 2 showed lower BMI, waist circumference, cholesterol LDL and LDL/HDL ratio than Group 1. Multiple linear regression, evidenced that in Group 2 a large vitamin $C$ intake was associated to lower LDL $(p=0.0015)$ while a high beta-carotene intake was correlated with higher HDL ( $\mathrm{p}=0.026)$. If they were assumed together we observed a correlation with a lower LDL/ HDL ratio, waist circumference and triglycerides $(p=0.005, p=0.033$, $\mathrm{p}=0.037$ respectively). Furthermore, the elevate anthocyanin consumption was correlated with lower triglycerides and waist circumference $(\mathrm{p}=0.01)$, but when anthocyanin was taken in association with vitamin $\mathrm{C}$ and beta-carotene there was a correlation with lower BMI also $(p=0.024)$. In conclusion, a diet with high intake of antioxidants is linked to a reduced $\mathrm{CD}$ risk parameters, with major influence of vitamin $\mathrm{C}$ and beta-carotene.

Intestinal transport of sardine (sardine pichardus) protein hydrolysate prepared from action of proteases from spent brewer's yeast (saccharomyces pastorianus) - assessment of antioxidant and ace-inhibitory activities

\section{Vieira $E^{\star}$ and Ferreira IMPLVO}

Department of Bromatology and Hydrology of Faculty of Pharmacy, University of Porto, Portugal, E-mail: elsavieiraf@gmail.com 
Sardine protein hydrolysate (SPH) with promising antioxidant and angiotensin-I-converting enzyme (ACE) inhibitory activities was studied for in vitro simulated gastrointestinal digestion and in vitro intestinal cell permeability. SPH was produced by incubation of sardine sarcoplasmic proteins (obtained from canning industry waste) with Saccharomyces yeast proteases (obtained from spent brewer's yeast) at an $\mathrm{E} / \mathrm{S}$ ratio of $0.27: 1(\mathrm{U} / \mathrm{mg}), \mathrm{pH} 6.0$ and $50{ }^{\circ} \mathrm{C}$ for 7 hours. This hydrolysate demonstrated in vitro ACE-inhibitory activity (IC50) of $236 \mu \mathrm{g}$ protein $/ \mathrm{mL}$ and FRAP value of $249 \mu \mathrm{M} \mathrm{TE} / \mathrm{mL}$. Both activities were enhanced by ultrafiltration through a $10 \mathrm{kDa}$ MWCO membrane, IC50 $182 \mu \mathrm{g} / \mathrm{mL}$ and FRAP value of $315 \mu \mathrm{M} \mathrm{TE} / \mathrm{mL}$. In vitro simulated gastrointestinal digestion increased $(\mathrm{p}<0.05)$ antioxidant capacity $(344$ $\mu \mathrm{M} \mathrm{TE} / \mathrm{mL}$ ); ACE-inhibitory activity of SPH remained unchanged (IC50 $187 \mu \mathrm{g} / \mathrm{mL}$ ), indicating that SPH peptides showed resistance to gastrointestinal proteases. Reverse phase chromatography analysis revealed that different peptides were responsible for antioxidant activity. A permeability assay using Caco- 2 cell monolayer and Caco2/HT29-MTX co-culture monolayer showed an intestinal transport of peptides that reached, respectively, $26.71 \%$ and $49.56 \%$ after $3 \mathrm{~h}$ at $37^{\circ} \mathrm{C}$. Paap values were $5.89 \times 10-6 \mathrm{~cm} \mathrm{~s}-1$ and $10.93 \times 10-6 \mathrm{~cm} \mathrm{~s}-1$, respectively. Mass spectrometry confirmed that the molecular weights of major peaks were $<10 \mathrm{kDa}$ regardless of ultrafiltration. For both models, intestinal transepithelial transport was efficient for some SPH peptides; permeates after $3 \mathrm{~h}$ at $37^{\circ} \mathrm{C}$ showed antioxidant activity but no ACE-inhibitory activity was detected. These results suggest that SPH prepared through Saccharomyces yeast proteases is a potentially bioavailable source of antioxidant peptides awaiting further in vivo study. Additionally studies are however needed to assess prospective antihypertensive effects. The reuse of these two agro-industrial by-products is advisable from both economic and environmental standpoints.

(-)-Carvone reduced intracellular triglyceride accumulation via activation of orlal in cultured hepatocytes

\section{Lee SJ*, Wu C, Jia Y and Kim Y}

Department of Biotechnology, Graduate School of Life Sciences and Biotechnology, BK21-PLUS, Korea University, Seoul 136-713, Republic of Korea, E-mail: junelee@korea.ac.kr

Aroma compounds in plants show biological activities interacting with cellular receptors and regulators. Here we show that (-)-carvone, a major aroma compound in spearmint reduces intracellular triglyceride concentrations. (-)-carvone is known as a ligand to a human olfactory receptor, OR1A1. In this study, OR1A1 expression was detected in HepG2 liver cells. OR1A1 activation by (-)-carvone increased the cyclic adenosine monophosphate (cAMP), but not intracellular $\mathrm{Ca} 2+$ concentration, thereby inducing protein kinase $\mathrm{A}$ (PKA) activity with subsequent phosphorylation of cAMP response element-binding protein (CREB) and upregulation of the CREBresponsive gene hairy and enhancer of split (HES)-1, a corepressor of peroxisome proliferator-activated receptor- $\gamma$ (PPAR- $\gamma$ ) in hepatocytes. In (-)-carvone-stimulated cells, the repression of PPAR- $\gamma$ reduced the expression of the target gene, mitochondrial glycerol-3-phosphate acyltransferase, which encodes a key enzyme involved in triglyceride synthesis. Intracellular triglyceride level and lipid accumulation were reduced in cells stimulated with (-)-carvone, effects that were diminished following the loss of OR1A1 function. These results indicate that OR1A1 may function as a non-redundant receptor in hepatocytes that regulates the PKA-CREB-HES-1 signaling axis and thereby modulates hepatic triglyceride metabolism.

\section{Date seeds: proximately composition and binding of bile acid} in vitro

\section{Gülden $G^{\star}$, Özge D, Sedat $S$ and H. İbrahim E}

Department of Food Engineering, University ofMersin, 33343, Mersin,Turkey,E-mail: gulgok33@gmail.com

Introduction: In the past few years there have been attempts to use agricultural by-products for potential health benefits in the diet formulation of human nutrition. Date seeds are by-products of date (Phoenix dactylifera) serve as good sources of dietary fiber compared with most fresh, dried fruits and their by-products. Increased intake of dietary fiber has been found to alter gut transit time, alert the colonic microflora, increase feces weight, decrease appetite effects, absorb toxins and bind bile acids. The bile acids are known amphiphilic steroids which are synthesized from cholesterol in the liver. The adsorption capacity of bile acids by dietary fiber is known regarding the mechanisms for the hypocholesteremic effect of dietary fiber and an increase in the fecal loss of bile acids. Objectives: The main objectives of this study were the determination of the proximate composition and the nutritional properties of date seed. Thus, protein, fat, soluble and insoluble dietary fiber, lignin, mineral compositions were analyzed. Additionally, total phenolic and antioxidant content and bile acid binding capacity of date seeds were also determined.Material and Methods: Date seeds were obtained from date palm three cultivars (Safawi, Sughai, Mabrum) grown in Madinah, Saudi Arabia. The dry matter, protein, lipid and ash content of date seeds were determined by using standard AOAC methods (AOAC, 2000). Soluble and insoluble dietary fiber was determined according to Prosky (1987) by using the Megazyme total dietary fiber analysis kit (Megazyme International Ireland Ltd, Wicklow, Ireland). The method given by Sayar (2005) was applied for the determination of bile acid binding capacity. Results: The protein, fat, ash and total dietary fiber contents of the date seeds varieties (Safawi, Sughai and Mabrum) were ranged from 5.42-5.60, 6.99-8.14, 0.81-0.97 and 85.43-86.41 g/100 g dry weight, respectively. The potassium concentration was the highest, followed in descending order by magnesium and phosphorus. Safawi date seed had the highest amount of total phenolics (48.32 mg GAE/g DM) and DPPH radical scavenging activity (6.17 mg TROLOX E/g DM). The bile acid binding activity of Safawi, Sughai and Mabrum date seeds were found to be 7.02, 7.67 and $6.96 \mathrm{mmol} / 100 \mathrm{~g}$ respectively. Conclusions: Date seeds can provide more dietary fiber in the diet than was previously believed. There could be relationship between BA binding ability and dietary fiber content according to these findings.However, micro and macro nutrients of date seeds to a wide range of products will contribute to the development of value-added or functional and technological foods that recently are in high demand.This confirms that the date seeds has great potential in food applications, especially in development of functional foods.

Coconut consumption and cardiovascular disease incidence, is there an association? A case control study in Sri Lankan population

\section{Athauda $\mathrm{LK}^{\star}$, Kasturiratne A and Wickremasinghe AR}

Department of Public Health, Faculty of Medicine, University of Kelaniya, Sri Lanka, E-mail: lathika@kln.ac.lk

Introduction \& objectives: Consumption of coconut and its products is reported to be associated with an increased risk of cardiovascular disease (CVD). This study was conducted to determine 
if there is an association between coconut consumption (oil and coconuts) and CVD incidence among Sri Lankans who are known to consume a large amount of coconut-based products. Methods: A hospital based case control study was conducted at the Colombo North Teaching Hospital, Ragama, Sri Lanka comprising 176 cases and 148 controls. Previously healthy patients admitted with a first incident Acute Coronary Syndrome (ACS) or a Cerebrovascular Event (stroke) were recruited as cases and patients with no previous history of CVD or diabetes mellitus were recruited from surgical wards as controls. There were 136 ACS and 40 Stroke patients as cases. An intervieweradministered questionnaire was used to estimate the average consumption of coconut (nuts and oil) and to obtain information on risk factors of CVD. Descriptive analysis and multiple logistic regression analysis were conducted using SPSS. Results: The mean $(+\mathrm{SD})$ age of cases and controls were $57.68+8.91$ and $56.05+10.02$, respectively $(\mathrm{p}=0.192)$. Mean $(+\mathrm{SD})$ coconut nut consumption per person per week was $1.97+0.81$ in cases and $2.11+1.01$ nuts in controls $(\mathrm{P}=0.66)$. Coconut oil use for cooking was $88.44+61.6 \mathrm{ml} /$ person/week in cases and $81.4+51.52 \mathrm{ml} /$ person/week in controls $(\mathrm{p}=0.175)$. $91.5 \%$ cases and $98 \%$ controls used coconut oil for cooking. Coconut oil use was protective for cases (OR:0.197, 95\% CI:0.052-0.744) while number of sedentary hours per day (OR:1.11, 95\% CI: 1.044-1.188) and family history of IHD (OR:2.293, 95\%CI:1.318-3.991) and stroke (OR:2.275, 95\%CI:1.268-4.082) were significant risk factors after adjusting for each other and for the amount of oil used per person per week, number of coconuts used per person per week, family history of NCD, being current smokers and consumers of alcohol. Conclusion: There was no evidence of an association between coconut consumption and incidence of cardiovascular disease or stroke. Use of coconut oil had a protective effect on the incidence of cardiovascular disease.

\section{Beneficial effect of low dose of ethanol on senescence- accelerated mice}

\section{Kato $\mathrm{N}^{1^{*}}$ and $\mathbf{I z u} \mathrm{H}^{2}$}

\section{${ }^{1}$ Hiroshima University, Japan}

${ }^{2}$ National Research Institute of Brewing, Japan, E-mail: nkato@ hiroshima-u.ac.jp

Accumulating epidemiological evidence suggests light to moderate alcohol intake confers multiple beneficial effects including risk reduction of several diseases such as type 2 diabetes, brain infarction, cognitive impairment, liver cirrhosis, cancers, etc (1). However, there is limited information regarding the effects of low dose of ethanol in animal studies. Recent our study has provided the evidence that $1 \%$ $(\mathrm{v} / \mathrm{v})$ ethanol intake in drinking water is beneficial for the liver function in rats fed a high-fat diet, but the $2 \%$ ethanol intake is less effective (2). This study was conducted to examine the effect of low dose of ethanol on the senescence of senescence-accelerated mouse 8 (SAMP8) marked by brain aging. Male SAMP8 mice (11 wk old) had free access to a commercial stock diet with drinking water containing 0,1 or $2 \%$ ethanol for $15 \mathrm{wk}$. The mean of total grading score of senescence, indicating the degree of senescence, in the $1 \%$ ethanol group was the lowest among the three groups until $26 \mathrm{wk}$, and the difference depended on the scores of their conditions of skin and hair, lordokyphosis, and behavior. Analysis using open field test showed the elevation of rearing counts in the $1 \%$ ethanol group, but not in the $2 \%$ ethanol group, implying the elevation of seeking behavior by the $1 \%$ ethanol intake. Furthermore, gene expression analysis indicated that the $1 \%$ ethanol intake markedly elevated the mRNA of brain alcohol dehydrogenase 1 , being responsible for the conversion of retinol to retinoic acid, a putative protective factor for brain diseases, but the $2 \%$ ethanol intake did not. We conclude the $1 \%$ ethanol intake is likely to be beneficial for SAMP8 mice. Taken together, our findings appear to be in favor of the putative protective effect of lower alcohol consumption against health problems.

\section{New trends in biological activity detection - relation to} nutrition and diagnostics

Krejčí $J^{1 *}$, O’Connel $M^{2}$, Stratmann $L^{3}$, Ventrubová $I^{1}$, Plička $R^{1}$, Krejčí $\mathrm{D}^{1}$ and Poláková $\mathrm{I}^{1}$

${ }^{1} B V T$ Technologies, a. s., Czech Republic
${ }^{2}$ Probe Scientific Ltd., United Kingdom
${ }^{3}$ PalmSens BV, The Netherlands, E-mail: jan.krejci@bvt.cz

Main Topics

1. Difference between activity and concentration. (Biological activity)

2. Time and its underestimation in online continuous analysis

3. Importance of sensors as a source of continuous data

4. Miniaturization of sensors. It decreases the measured system changes. It decreases the time resolution of the measurement.

5. Electrochemical sensors

6. Biosensors

7. Microdialysis and body interfaces

8. Practical examples (continuous glucose monitoring, continuous lactate monitoring, continuous dopamine monitoring, activity of catalase as antioxidant marker, antioxidant activity of wine, traces of algae inhibitors)

The new technologies (microsensors, microfluidics) enable to monitor the physiological processes in more reliable conditions (wearable devices) and with better time resolution. It brings new insights in many biochemical processes. The first steps were demonstrated by examples in the lecture.

\section{Dioxins in food of lifestock-farming?}

\section{Schiefer $\mathrm{HP}^{\mathbf{1}^{*}}$ and Sandra $\mathrm{P}^{2}$}

\section{${ }^{1}$ Independent consultant, Ternitz, Austria}

${ }^{2}$ Ghent University, Belgium, E-mail: hanspeterschiefer@gmail.com

This is all about falsification or/and manipulation of chemical analytical results in food:

The so called Dioxin-scandal(TCDD=Tetrachlorodibenzodio xin) 1999 in Belgium, followed by the Irish Dioxin scandal 2008, then by the German Dioxin-scandal in 2011 and 2014 the so-called HCB(Hexachlorobenzene)- scandal in Austria are typical examples of disinformation or telling only half the truth. To avoid the bitter truth of a high PCBs (Polychlorinated Biphenyl)-concentration the burden was shifted to the 10.000-less concentrated TCCDs or TCDFs. Prof.Pat Sandra from Belgium could prove already in 1999 that the so-called Dioxin-scandal was in fact a PCB-scandal, but neither the German governmental institutions nor the Austrians have learned, instead both tried like the Belgian government to avoid any publicity, because they feared the breakdown of the meat industry or in case of 
the HCBs in Austria the controlling authorities and the cementfactory processmanagement did not want the people realizing that they did a sloppy job.

Is manipulation ethical? Even when it forces people to do the right things or avoids panicking? For more info please refer to the handout material,which I will provide.

\section{P01. Determination of vitamin B1, vitamin B2, niacin and trace metals in pigmented thai rice varieties}

\section{Jansom $\mathrm{C}^{\star}$ and Lerdvuthisophon $\mathbf{N}$}

Faculty of Medicine, Thammasat University, Pathumthani, Thailand, E-mail: jchalerm@hotmail.com

There are many indigenous varieties of rice in Thailand, and some have been commercially successful. They are exported to many countries because of their higher yield, disease resistance, ability to be harvested throughout the year, especially palatability and nutritional value. This study determined the contents of vitamin B1, vitamin B2 and niacin in twenty varieties of half-milled rice. The levels of essential elements were determined by atomic absorption spectrometer (AAS) and mercury was determined using an in-house developed cold vaporAAS method. Normal and glutinous rice varieties of purple to black $(n=9)$, orange to deep red $(n=9)$ and white pigmentation $(n=2)$ were collected from various rice research centers throughout Thailand. All varieties of rice had high contents of vitamin B1, especially Hom-Dang Sukhothai 1 and Khao Dawk-Mali 105 varieties. Most kinds of rice had low levels of vitamin B2, except PSL00255-4-4-5R and Hom-Dam Sukhothai 2 varieties had rather high contents of vitamin B2. All rice had a small amount of niacin (less than 15 microgram per gram). The average contents of vitamin B1, vitamin B2 and niacin were no difference between pigmented, and no difference between normal and glutinous rice. Most types of rice showed high levels of Fe, Mn and Zn, especially Hom-Phukhiew and Dang-Muenglong varieties, while all had low levels of $\mathrm{Cr}, \mathrm{Cu}$ and Se. Eleven varieties of rice had trace levels of mercury (less than 0.5 microgram per gram) and in nine kinds mercury was undetectable (lower than the detection limit $=0.0034$ microgram per gram).

P02. Total phenolics compound, total flavonoids, tocopherols and gamma-oryzanol in pigmented thai rice varieties

\section{Jansom $\mathrm{V}^{\star}$ and Lerdvuthisophon $\mathrm{N}$}

Faculty of Medicine, Thammasat University, Pathumthani, Thailand, E-mail: bum_vanida@hotmail.com

There are numerous traditional rice varieties and rice specialists are producing many improved varieties. However, only a few varieties can be registered with the National Thai Department of Rice due to their unique hereditary characteristics and quality. Currently, the high bioactivities of some varieties are attracting interest among researchers and consumers. This study determined the contents of total phenolics compound, total flavonoids, vitamin $\mathrm{E}$ isomers and gamma-oryzanol in fifteen varieties of half-milled rice. The improved pigmented $(n=12)$ and white $(n=3)$ rice varieties were collected from various rice research centers throughout Thailand. The total phenolic content in pigmented varieties was significant higher than white. The highest total phenolic content was found in black rice (Niew-Dam Leum Pua) with $288 \mathrm{mg}$ (gallic acid equivalent) per $100 \mathrm{~g}$ (half-milled rice). The high content of total phenolic was found in black rice (Hom-Dam Sukhothai 2) and in red rice (Hom-Mali Dang). All pigmented varieties of rice had high contents of total flavonoid, especially Hom-Dang and NiewDam Leum Pua varieties. The total flavonoid content in black rice was significant higher than red rice, where as white rice varieties had low levels of total flavonoid. All varieties of rice showed high contents of alpha-tocopherol while they had low amounts of beta, gamma, deltatocopherols and tocotrienols. Gamma-oryzanol was noticeably higher in black rice and some kinds of deep red rice. The remaining varieties of deep red, purple and red rice had a lower amount of gamma-oryzanol, while orange and white rice had the lowest content.

P03. Effects of 6-month weight loss new programme on anthropometric measurements and biological profile

\section{Hamouda $\mathrm{A}^{1^{*}}$ and Abou El Noeman $\mathrm{S}^{2}$}

${ }^{1}$ Department of Biochemistry, Faculty of Science, Alexandria, Egypt

${ }^{2}$ Department of Medical Biochemistry, Faculty of Medicine, University of Tanta, Egypt, E-mail: asmaakingdom1@yahoo.com

Introduction: Obesity has become a leading global health problem owing to its strong association with a high incidence of diseases. Obesity results from the complex interaction of environmental factors that act on a genetic background. Obesity is a pathological condition in which excess body fat has accumulated to the extent that it may have an adverse effect on health. Treatment of obesity includes a two-step: assessment and management. Assessment includes determination of the degree of obesity and overall health status. Management involves not only weight loss and maintenance of body weight but also measures to control other risk factors. Aim: To investigate effect 6 months safe weight loss programme on anthropometric measurements and biological and metabolic profiles in obese patients. Subjects and Methods: 35 obese patients were enrolled in the present study: 20 female, 15male. Participants underwent a comprehensive series of biochemical, anthropometrical, physical, and nutritional prior to treatment- at baseline and after the 6-month of obesity treatment program. 6 months weight loss programme:

- Diet plan intervention in small groups

- In-person training and individual diet plan intervention

Results: There is significant reduction of weight and high improvement in laboratory parameters. Conclusions: Our data showed that there an improvement in weight loss. These finding may be important for controlling obesity-related co-morbidities. It would appear that moderate weight loss of 8-10\% observed in our study resulted in significant improvements in laboratory parameters.

P04. Short and long term effects of pomegranate extracts (punica granatum) on apoptosis in rat kidney induced by diethylnitrosamine and phenobarbital

\section{Hamouda $\mathrm{A}^{\star}$ and Shaban $\mathbf{N}$}

Biochemistry Department, Faculty of Science, Alexandria University, Alexandria, Egypt,E-mail: asmaakingdom1@yahoo.com

Pomegranate (Punica granatum L.) has strong anti-inflammatory, antioxidant, anti-obesity, and anticancer effects. The effect of different pomegranate extracts, peel extract (PE), seed oil extract (SOE), and pomegranate juice extract (PJ) on levels of kidney caspase-3, DNA fragmentation (DNAF) and kidney function tests in rats treated and untreated with diethyl nitrosamine (DEN) and Phenobarbital(PB) during short (35 days) and long (154 days) period was studied. Injected of rats with DEN and PB caused an increased in the levels of DNAF, 
caspase- 3 and kidney function tests, compared to the control in both period of study. Treatment of rats with PE, SOE, PJ pre, during, and post DEN and PB administration improved kidney function and decreased the levels of DNAF, and caspase- 3 activities compared to the DEN group in both period of study, indicates that PE, SOE, PJ reduced and treatment apoptosis induced by DEN and $\mathrm{PB}$. Treatment of healthy rats with PE, SOE, and PJ only for 35 days not increased kidney function or induced apoptosis for kidney tissues. Treatment with PJ alone in healthy kidney induced apoptosis which was higher than that induced by SOE and PE in case of long period study, this mean that fresh fruit or pomegranate juice safe for healthy in general at harvesting season only.

P05. Antibacterial activity of thai medicinal plant extracts against oral and gastrointestinal pathogenic bacteria and prebiotic effect on the growth of lactobacillus acidophilus

\section{Nanasombat $S^{\star}$, Nattakorn $K$, Ritcharoon B and Sukcharoen $P$}

Department of Biology, Faculty of Science, King Mongkut's Institute of Technology Ladkrabang, Bangkok 10520, Thailand, E-mail: snanasombat@gmail.com

Gastrointestinal tract infections are the most common gastrointestinal disorders. Pathogenic bacteria related to the pathogenesis include Porphyromonas gingivalis (an oral pathogen), Helicobacter pylori (a stomach pathogen) and other pathogenic bacteria, Bacillus cereus, Escherichia coli, Enterobacter aerogenes, Listeria monocytogenes, Salmonella Rissen, Salmonella Typhimurium, Staphylococcus aureus and Yersinia enterocolitica. To alleviate these problems, antibacterial activity of plant extracts against these bacteria should be explored. The 25 crude methanolic extracts of Thai medicinal plants were tested for their antibacterial activity against the bacterial strains as mentioned using agar diffusion test and minimum inhibitory concentration (MIC) determination. Their phytochemicals and prebiotic propertieswere also determined. Rhubarb (Rheum palmatum) root, wild turmeric (Curcuma aromatica) rhizome, aleppo oak (Quercus infectoria) gall and ringworm bush (Cassia alata) stem extracts

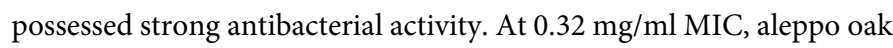
and wild turmeric extracts inhibited the growth of Y. enterocolitica, while rhubarb, wild turmeric,aromatic ginger(Kaempferia galanga) rhizome and ringworm bush extracts inhibited $\mathrm{P}$. gingivalis growth. The aleppo oak gall extract had highest phenolics and tannins (672.13 mg gallic acid equivalents (GAE)/g extract and $884.79 \mathrm{mg}$ tannic acid equivalents (TAE)/g extract, respectively), whereas the copper pod (Peltophorum pterocarpum) stem bark extract had highest flavonoids $(5,293.60 \mathrm{mg}$ quercetin eqivalents (QE)/g extract). Five plant extracts with high water soluble carbohydrate were tested for their prebiotic properties. Their indigestible polysaccharide content and stimulatory effect on the growth of Lactobacillus acidophilus in MRS broth were determined. Of all, mangosteen (Garcinia mangostana) fruit peel and gac (Momordica cochinchinensis) rootextracts had high indigestible polysaccharide contents, and exhibited good stimulatory effect to the growth of Lactobacillus acidophilus. In conclusion, the rhubarb root, wild turmeric rhizome, aleppo oak gall and ringworm bush stem extracts could be used in combination with mangosteen fruit peel and gac root extracts for treatment of gastrointestinal tract infections.
P06. Effect on organoleptic properties of biscuits fortified with fenugreek seed germinated until 5 days

\section{Aljumayi $\mathbf{H}^{\star}$}

\section{Taif University, Saudi Arabia, E-mail: drhuda2008@gmail.com}

The objective of this study was to evaluate the effect of fortification of biscuits with $10 \%, 20 \%$ and $30 \%$ of fenugreek seed that germinated for 2, 3, 4, and 5 days. The organoleptic properties results showed that a maximum of $10 \%$ fenugreek flour germinated for 2 and 3 days can be incorporated to prepare acceptable quality biscuits. However, 10 and $20 \%$ germinated fenugreek flour is preferred when increasing germination time up to 4 and 5 days. The increased in the germination time and percentage of adding germinated fenugreek flour to wheat flour altered the contents of protein and dietary fiber. These biscuits can be safely stored in polypropylene bags up to 2 months without altering their organoleptic properties.

\section{P07. Development of a nutritional wellness programme for} staff

\section{Devar $\mathrm{S}^{1^{*}}$ and Napier $\mathrm{C}^{2}$}

${ }^{1}$ Durban University of Technology, South Africa

${ }^{2}$ University of Mpumalanga, South Africa,E-mail: siveshnee.devar@ ump.ac.za

Background: The rate of absenteeism and prevalence of NCDs in South Africa is extremely high. This is consistent with other educational institutions and workplaces around the globe. In most cases the absence of health and the presence of one or more non communicable diseases coupled with the lack of physical exercise is a major factor in absenteeism. Absenteeism at the workplace comes at a huge cost to the employer and the country as a whole. Aim: Findings from this study was to develop a suitable nutritional wellness programme for the workplace.Methodology: A needs analysis in the form of a 24-hour recall, food frequency, health and socio demographic questionnaires was undertaken to determine the need for a wellness program for the institution. Anthropometric indices such as BMI, waist circumference and blood pressure were also undertaken to determine the state of health of the staff.Results: This study has found that obesity, central obesity, hypertension as well as deficiencies in nutrients and minerals were prevalent in this group. Fruit and vegetable consumption was also below the WHO recommendation. This study showed a link between diet, physical activity and diseases of lifestyle. There were positive correlations between age and systolic blood pressure, waist circumference and systolic blood pressure, waist circumference and diastolic blood pressure and waist-to-height ratio and BMI.Conclusion: The results indicated the need for immediate intervention in the form of a wellness program. Nutrition education is important for both the workplace and out. Education and knowledge are important factors for lifestyle changes. The proposed intervention is aimed at improving presenteeism and decreasing the incidence of non- communicable diseases. Presenteeism and good health are important factors for quality education at all educational institutions.

P08. Effect of essential oils of marjoram and rosemary on beef patties manufactured with mechanically deboned poultry meat

\section{Moustafa $\mathrm{H}^{\star}$ and Mohamed $\mathrm{H}$}

Department of Food Hygiene and Control, Faculty of Veterinary 
Medicine, Cairo University, Giza, Egypt, E-mail: hayamansour@ hotmail.com

The effect of addition of essential oils of marjoram (Origanum marjorana L.) and rosemary (Rosmarinus officinalis L.) at concentration of $200 \mathrm{mg} / \mathrm{kg}$ to beef patties formulated with $20 \%$ mechanically deboned poultry meat (MDPM) was evaluated. The proximate composition, lipid oxidation, sensory characteristics and Psychrotrophic counts of beef patties manufactured with 20\% MDPM (percentage of lean portion) were assessed during frozen storage at $-18{ }^{\circ} \mathrm{C}$. Beef patties formulated with MDPM showed significant $(\mathrm{P}<0.05)$ reduction in protein content and significant $(\mathrm{P}<0.05)$ increase in fat content. Incorporating of essential oils of marjoram and rosemary at level of $200 \mathrm{mg} / \mathrm{kg}$, significantly $(\mathrm{P}<0.05)$ reduced the TBARS of beef patties formulated with or without MDPM. Moreover, the TBARS of formulas prepared with natural herbal essential oils remained significantly $(\mathrm{P}$ $<0.05)$ lower during frozen storage for 3 months. TBARS values of formulas prepared with essential oils of marjoram and rosemary were significantly $(\mathrm{P}<0.05)$ lower than those of formulas prepared with the synthetic antioxidant BHT. The addition of natural herbal extracts to beef patties did not significantly $(\mathrm{P}>0.05)$ change the $\mathrm{pH}$ values of all formulas after preparation and during frozen storage for 3 months. Moreover, antioxidants incorporation did not significantly $(\mathrm{P}>0.05)$ reduce the psychrotrophic counts in all beef patties during frozen storage. A significant $(P<0.05)$ increase in the sensory scores and overall acceptability of beef patties with added natural herbal extracts during frozen storage period was observed. Overall, the study indicated the potential use of natural herbal essential oils to protect against lipid oxidation and improve the sensory attributes of beef patties formulated with MDPM. Furthermore, the superiority of essential oils of natural herbal extracts at level of $200 \mathrm{mg} / \mathrm{kg}$ to synthetic antioxidants was observed.

P09. The effect of resorcinolic lipids and acetylcholinesterase inhibitors on the activity of different molecular forms of acetylcholinesterase

\section{Stasiuk $M^{\star}$, Piwoni A, Prokopowicz M and Kozubek A}

University of Wroclaw, Faculty of Biotechnology, ul. Joliot-Curie 14a, 50-383 Wroclaw, Poland, E-mail: stasiuk@ibmb.uni.wroc.pl

Acetycholinesterase (AChE) inhibitors are commonly used to elevate acetylcholine levels in patients with Alzheimer's disease. Resorcinolic lipids were reported to have various biological activities which suggest their involvement in the regulation of cell and organism metabolism. These activities include the ability to modulate AChE activity. Our experiments indicated that alkylresorcinols with a long side-chain in the phospholipid bilayer (preincorporated in liposomes or after incorporation into a biological membrane) reduce the effectiveness of three different molecular forms of AChE inhibition by donepezil, physostigmine and galantamine. This may have therapeutic significance. However, alkylresorcinols added in a methanol solution (i.e., in the free form) act cooperatively with the above-mentioned inhibitors to cause complete enzyme inhibition.

P10. The analysis of anti-oxidative capacity of water-soluble substances in mulberry leaves and fruits

$\operatorname{Kim} \mathbf{H}^{1^{*}}$, Kim $S^{1}$, Seok $Y^{2}$, Seo $S^{3}$ and Sung $G^{1}$

${ }^{1}$ National Academy of Agricultural Science, Korea

${ }^{2}$ Gangwon-do Agricultural Product Registered Seed Station, Korea
${ }^{3}$ Chungcheongnam-do Sericultural and Entomology Experiment Station, Korea, E-mail: hyunbok@korea.kr

The mulberry leaves, root bark, and twigs have long been used in Chinese medicine to treat fever, protect the liver, improve eyesight, strengthen joints, facilitate discharge of urine, and lower blood pressure. Different parts of the mulberry, from the root bark to the leaves, have been extensively investigated for their various health benefits, including antioxidative, hypolipidemic, antihyperglycemic, and antiatherogenic effects. Previous studies have indicated that mulberry fruits and leaves exhibited significant scavenging effects on free radicals and protected low-density lipoprotein against oxidative damage. The reports suggested that polyphenols and flavonoids present in mulberry contribute to these health. Especially, mulberry leaves containing many natural components are considerable resource for natural antioxidants. The antioxidant capacity of mulberry leaves was investigated with minilum L-100 device and ARAW-KIT (anti-radical ability of watersoluble substance), in comparison to the ascorbic acid. The appropriate conditions for pre-treatments of mulberry leaves were $80 \% \mathrm{MeOH}$, $30 \mathrm{sec}$. vortex mixing. The antioxidant capacity of 16 varieties was $3303.4 \mathrm{nmol}$ (ascorbic acid equivalents) at opening stage of five leaves in spring. The highest stage of antioxidant capacity $(3708.0 \mathrm{nmol})$ and yield rate was just before the coloration stage with anthocyanin in fruits, whereas the lowest stage was middle of June $(2231.6 \mathrm{nmol})$ and about two months growing stage after summer pruning $(2064.6 \mathrm{nmol})$. But after summer pruning, the antioxidant capacity of mulberry leaves increased gradually until just before fallen leaves stage.

P11. Plasma glutamine and cystine levels are decreased and correlated with endomysial antibody in children with celiac disease

Sevinc $\mathrm{E}^{1^{*}}$, Sevinc $\mathrm{N}^{2}$, Sezgin $\mathrm{G}^{1}$, Akar $\mathrm{H}^{3}$, Arslan $\mathrm{D}^{1}$ and Kendirci $\mathrm{M}^{4}$

${ }^{1}$ Erciyes University, Pediatric Gastroenterology Kayseri, Turkey

${ }^{2}$ Erciyes University, Public Health Kayseri, Turkey

${ }^{3}$ Erciyes University, Pediatric Allergy Kayseri, Turkey

${ }^{4}$ Erciyes University, Pediatric Metabolism Kayseri, Turkey, E-mail: dr.eylemsevinc@gmail.com

Background: Glutamine, a nonessential amino acid, improves intestinal mucosal regeneration and absorption. Cystine is the precursors of glutathione, which is vital for antioxidant reactions in the body. The first aim of the study is to measure the glutamine and cystine levels in children with celiac disease (CD) and compare them with the controls. The second aim of the study is to investigate whether these amino acids correlates endomysial antibody (EMA) or not.

Materials and methods: Fifty children with $\mathrm{CD}$ were compared to 50 healthy age and sex matched normal children as control. The plasma glutamine and cystine levels of the children were measured by using tandem mass spectrometry.

Results: The plasma glutamine and cysteine were significantly $(\mathrm{p}<0.05)$ lower in coeliac group ( $808 \mathrm{vs} 19 \mu \mathrm{mol} / \mathrm{l}$, respectively) than in controls $(870 \mu \mathrm{mol} / \mathrm{l}, 48.5 \mu \mathrm{mol} / \mathrm{l})$. The plasma glutamine and cystine levels were lower $(\mathrm{p}<0.05)$ in EMA-positive $(796.5 \mathrm{vs} 18 \mu \mathrm{mol} / \mathrm{l} ; \mathrm{n}=42)$ compared with EMA-negative (928 vs $31.5 \mu \mathrm{mol} / \mathrm{l} ; \mathrm{n}=8$ ) patients with $\mathrm{CD}$. The plasma glutamine ( $928 \mathrm{vs} 870 \mu \mathrm{mol} / \mathrm{l}, \mathrm{p}>0.05$ ), and cystine (31.5 vs $48.5 \mu \mathrm{mol} / \mathrm{l}, \mathrm{p}>0.05$ ) levels were not significantly different between EMA-negative patients with $\mathrm{CD}$ and healthy controls. No statistically significant differences were seen for age, anthropometric 
characteristics, serum total protein or albumin $(\mathrm{p}>0.05)$.

Conclusions: Our study demostrated the plasma glutamine and cystine levels were significantly lower in children with $\mathrm{CD}$, and also the low levels of these amino acids negatively correlated to serum EMA level. Further investigation are needed whether plasma cystine and glutamine levels measurements help to reflect of the intestinal oxidative damage and for assessment compatibility of gluten free diet in the children with CD.

\section{P12. Hydroxy radical scavenging activities of cerials}

\section{Ukai $M^{\star}$}

Hokkaido University of Education, Japan, E-mail: ukai.mitsuko@h. hokkyodai.ac.jp

The radical scavenging activity against hydroxy radical was examined using a new reagent 5-(2,2-dimethy-1,3-propoxycyclophosphoryl)5-methyl-1-pyrroline N-oxide (CYPMPO). CYPMPO, colorless crystalline, is very soluble in aqueous solution. For careful detection of spin adducts, we used borosilicate ESR flat cell. We examined cereal specimens of wheat, rice and barley. We generated very pure hydroxy radical by illuminating phosphate buffer solution containing hydrogen peroxide, diethylene-triamine-pentaacetic acid (DTPA), and CYPMPO. The spin adducts by CYPMPO were sensitive and very stable. CYPMPO successfully trapped hydroxy radical in the specimens. The scavenging activity against hydroxy radical was measured by L-ascorbic acid or mannitol equivalents. We concluded ESR spin trap method with CYPMPO is useful for determine the radical scavenging activities against hydroxy radical of cereals.

\section{P13. Radical scavenging activities of plant food of multiple radicals}

\section{Kameya $\mathbf{H}^{\star}$}

NARO, Nation Food Research Institute, Japan, E-mail: hkameya@ affrc.go.jp

The multiple radical scavenging activity were examined using a new reagent 5-(2,2-dimethy-1,3-propoxycyclophosphoryl)-5-methyl1-pyrroline $\mathrm{N}$-oxide (CYPMPO) and 2,2,6,6-tetramethylpiperidine 1-oxyl (TEMPO). For careful detection of spin adducts, we used borosilicate ESR flat cells. We examined plant specimens from teas, vegetables and fruits. We generated very pure hydroxyl radical, alkyl-oxy radical, superoxide radical, singlet oxygen by illuminating phosphate buffer solution containing radical generation reagent and CYPMPO or TEMPO with $\mathrm{Hg}$-Xe arc lamp. The spin adducts by CYPMPO or TEMPO were sensitive and very stable. CYPMPO and TEMPO successfully trapped each pure radicals in the plant extracts. We concluded ESR spin trap method is useful for determine the multiple radical scavenging activities of plant food.

P14. Efficacy evaluation of dandropanax morbifera extracts on indomethacin induced gastric mucosal damage in rats

Lee WK ${ }^{1,2^{\star}}$, Park HH ${ }^{1,2}$ Ko TH $^{2,3}$, Thuy Linh TT $^{2,3}$ and Pyao $\mathrm{Y}^{2,3}$

${ }^{1}$ Laboratory of Developmental Genetics, Department of Biomedical Sciences, South Korea

${ }^{2}$ School of Medicine, Inha University, Incheon 400-712, South Korea

${ }^{3}$ Laboratory of Developmental Genetics, Department of Biomedical Sciences, South Korea, E-mail: wklee@inha.ac.kr
Dandropanax morbifera Lev. is belongs to araliaceae and can be found along the southern coast of South Korea and islands around. It has been shown anti-oxidation function as well as inhibition of liver damage. But its efficacy does not evaluated and confirmed on scientific methods such as in vivo approach with animal models. In the present study we have investigated the efficacy of Dandropanax morbifera extracts on its anti-ulcer effect using indomethacin induced gastric mucosal damage in rats. We divided the animals into 5 groups as follows: (1) vehicle control, (2) $25 \mathrm{mg} / \mathrm{kg}$ indomethacin, (3) $25 \mathrm{mg} /$ $\mathrm{kg}$ indomethacin $+1.7 \mathrm{ml} / \mathrm{kg}$ Dandropanax morbifera extracts, (4) 25 $\mathrm{mg} / \mathrm{kg}$ indomethacin $+8.3 \mathrm{ml} / \mathrm{kg}$ Dandropanax morbifera extracts, (5) $25 \mathrm{mg} / \mathrm{kg}$ indomethacin $+100 \mathrm{mg} / \mathrm{kg}$ lansoprazole.In groups 2,3 , 4 and 5, indomethacin was given at 30 minutes after Dandropanax morbifera extracts administration. $25 \mathrm{mg} / \mathrm{kg}$ indomethacin was given to all rats $(n=10)$ by oral gavage in $3 \%$ sodium bicarbonate. Seven hours after, the entire rats were sacrificed and confirmed gastrointestinal erosions and ulcers. Dandropanax morbifera extracts treatment before administration of indomethacin resulted in a decrease score in gastric damage areas in the entire group treated $(\mathrm{P}<0.05)$. It could be confirmed $\mathrm{H} \& \mathrm{E}$ staining with gut mucosal damage as well. And also, iNOS expression was decreased in Dandropanax morbifera extracts treatment group which is expressed very much in G2 group as an inflammation control. Our data suggests that Dandropanax morbifera extracts functions the protective effect on indomethacin-induced gastric mucosal damage.

P15. Evaluation of caffeic, chlorogenic, rosmarinic acid contents and antioxidant activity of some lamiaceae species

Benedec $D^{1 *}$, Oniga $\mathrm{I}^{1}$, Olah $\mathrm{NK}^{2}$, Raita $\mathrm{O}^{3}$, Hanganu $\mathrm{D}^{1}$ and Tiperciuc B ${ }^{4}$

${ }^{1}$ Luliu Hatieganu University of Medicine and Pharmacy, Department of Pharmacognosy, 12 I. Creanga Street, Cluj-Napoca 400010, Romania

${ }^{2}$ Department of Therapeutical Chemistry, Pharmaceutical Industry and Pharmaceutical Biotechnologies, Vasile Goldis Western University of Arad, 86 L. Rebreanu Street, Arad and Plantextrakt, 3462 Radaia, Cluj, Romania

${ }^{3}$ Department of Physics of Nanostructured Materials, National Institute for Research and Development of Isotopic and Molecular Technologies 65-103, Donath Street, 400301, Cluj-Napoca Romania

${ }^{4}$ Luliu Hatieganu University of Medicine and Pharmacy 12 I. Creanga Street, Cluj-Napoca 400010, Romania,E-mail: dani_67ro@ yahoo.com

In this investigation, six indigenous species of Lamiaceae family, namely Rosmarinus officinalis, Salvia officinalis, Melissa officinalis, Origanum vulgare, Ocimum basilicum and Hyssopus officinalis, were screened for to evaluate the caffeic, chlorogenic and rosmarinic acid contents and the antioxidant activity. HPLC analysis of the ethanolic extracts revealed the presence of rosmarinic acid in the largest amount in O. vulgare and M. officinalis ( 3.29 and $2.97 \mathrm{mg} / \mathrm{mL}$, respectively) and in the lowest in $\mathrm{H}$. officinalis $(0.31 \mathrm{mg} / \mathrm{mL})$. The results showed that among the analyzed plants, the high caffeic acid content was found in R. officinalis $(0.76 \mathrm{mg} / \mathrm{mL})$, Chlorogenic acid was determined only in O. basilicum $(0.32 \mathrm{mg} / \mathrm{mL})$.The extracts were screened for antioxidant activities using four in vitro assay models: DPPH radical scavenging assay, ferric reducing ability plasma (FRAP) method, cupric reducing antioxidant capacity (CUPRAC) assay, and an electron paramagnetic resonance (EPR) radicals' detection. O. vulgare and $\mathrm{M}$. officinalis extracts exhibited the highest antioxidant capacity, in good agreement 
with the phenolic acids content.The obtained results may indicate that these phenolic acids content could be used as a taxonomic marker and confirm, at the chemical level, differences among species.

P16. Dietary diversity of hiv-infected people from rural and urban communities in the free state, South Africa

Walsh $\mathrm{CM}^{{ }^{*}}$, Pienaar $\mathrm{M}^{1}$ and Van Rooyen $\mathrm{FC}^{2}$

${ }^{1}$ Department of Nutrition and Dietetics, University of the Free State, South Africa

${ }^{2}$ Department of Biostatistics, University of the Free State, South Africa,E-mail:walshcm@ufs.ac.za

Introduction: A diet that is sufficiently diverse is likely to reflect nutrient adequacy and aid in health promotion and disease prevention. Objective: The objective of this study was to determine dietary diversity of people from rural and urban communities in the Assuring Health for All (AHA) study that was undertaken in 991 participants from the rural and urban Free State province in South Africa.

Method: Adults between 25 and 64 years were eligible to participate. A 24-hour recall of food intake was completed during individual interviews with all adults and venous blood samples were drawn to determine HIV status. A household dietary diversity score (DDS) was calculated from the intake of 12 possible food groups to classify dietary diversity as low ( 3 or less), medium (4 and 5) or high (6 or more). Blood samples were analysed in an accredited laboratory using standard techniques. Results: Of the 567 rural participants, 97 (17.1\%) were HIV-infected. Of the 424 urban participants, 172 (40.6\%) were HIV-infected. Most meals typically consisted of starches, e.g. maize meal porridge and bread. Of particular concern was the low intake and diversity of vegetables and fruits and milk in all groups and the low intake of flesh meats in the urban group. Intake of fats and oils; sweets; and spices, condiments, and beverages tended to improve DDSs, without necessarily depicting healthy food choices. One out of five participants had low to medium DDSs, but no significant differences in the DDSs of HIV-infected and HIV-uninfected participants occurred in rural and urban areas.

Conclusion: Interventions aimed at improving nutritional status of HIV-infected persons should not only aim at improving dietary diversity, but also focus on encouraging healthy food choices.

P17. Study on the correlation between instant noodle intake and cardiometabolic risk factors of healthy Korean university students

\section{$\operatorname{Kim} S \boldsymbol{J}^{*}$}

Department of Family Medicine, Seoul National University College of Medicine, E-mail:soojin.84@gmail.com

Background: South Korean people have consumed over 3.6 billion packages of instant noodles in 2013, which was the highest figure in the globe. According to the 2012 Korean National Health Statistics Report, the early mortality during the age from the twenties to the forties caused by cardiovascular disease has been increased. This study is aimed to define the correlation between instant noodle intake and cardiometabolic risk factors of healthy young men and women who are 18 to 29 years old. Methods: A total of 3,876 subjects with the age of 18 to 29 years were selected from the same university. The subjects had gone through the same regular physical exam in 2013 and had no history of chronic disease. 2013 web survey and health check-up data including questionnaires on eating habits and health behavioral habits have been used for this study. Also, serum cholesterol subfractions, fasting glucose, blood pressure and physical data including body weight and waist circumference were measured.

Results: BMI, abdominal circumference, blood pressure, fasting glucose level, low HDL level, triglyceride level, number of metabolic syndrome components significantly increased according to the higher frequency of instant noodle intakes. Even after the control of potential confounding factors (health behavioral variables, other food intake variables), BMI, abdominal obesity, systolic blood pressure, diastolic blood pressure and serum triglyceride level have been statistically increased in accordance with the frequency. Conclusions: This study suggests that the frequency of instant noodle intakes is significantly correlated to the cardiometabolic risk factors of healthy 18-29 year olds.

P18. Immunomodulatory effects of lipopolysaccharide from the butyrivibrio group bacterium isolated from the goat rumen

\section{Asanuma $\mathbf{N}^{*}$}

Department of Life Science, College of Agriculture, Meiji University, Japan,E-mail:asanuma@meiji.ac.jp

Some strains of lactic acid bacteria are utilized as probiotics for health benefits on the host. Such bacteria have been shown to contribute to the host immune homeostasis. The genus Butyrivibrio is one of mammalian intestinal bacteria, and some strains of them might have the potential to improve immune responses. In this study, we aimed to isolate the Butyrivibrio strain that has the immunomodulatory effects by evaluating the cytokine generating ability and investigating the effect of oral administration of the selected isolate in mice. Several strains of Butyrivibrio were isolated from the goat rumen. The cells of isolated bacteria were heated and freeze-dried. Mouse splenocytes were cultured in the presence of the isolated bacteria, and then the culture supernatants were collected to measure cytokines produced by ELISA. When mouse splenocytes were cultured in the presence of isolated bacteria, two isolates showed which had the high ability to stimulate IL-12 generation. The phylogenetic analysis of $16 \mathrm{~S}$ rRNA sequence revealed that the two isolates are placed in the cluster of Butyrivibrio species. One of the two isolates stimulated IFN-gamma generation in the splenocyte culture. The lipopolysaccharide (LPS) fraction of this isolate had effects that are comparable to those of freeze-dried cells, suggesting that LPS is responsible for the immunomodulatory effects of this isolate. When mice were orally administrated the LPS fraction of the isolate, NK cell activity was increased in the spleen. The production of IFN-gamma, but not IL-4, in cultured splenocytes was augmented by administration of LPS. It is likely that the isolated Butyrivibrio species has immunomodulatory effects such as enhancing NK cell activity via its LPS.

P19. Investigation of prediabetes and dyslipidemia risk indicators in overweight and obese women

\section{Hizli $\mathrm{H}^{1 \star}$ and Azezli $\mathrm{A}^{2}$}

${ }^{1}$ Department of Nutrition and Dietethic, Istanbul Medipol University, Turkey

${ }^{2}$ Department of Internal Medicine, Istanbul University, Istanbul Faculty of Medicine, Turkey, E-mail: hhizli@medipol.edu.tr

Introduction: The prevalence of obesity is increasing rapidly in recent years. Rapid increase in obesity and associated diseases like 
cardiovascular disease and type 2 diabetes mellitus in many societies revealed contemporary terminology such as "prediabetes" and "dyslipidemia" that refer pre-clinical disorders. The aim of this study was to investigate the presence of prediabetes and dyslipidemia risk indicators in overweight and obese women.

Method: This retrospective study included 493 female patients with a mean age of $40.6 \pm 13.4$ years. Height, weight, waist and hip circumference, systolic and diastolic blood pressure were measured. Biochemical measurements were taken. Body mass index (BMI) and HOMA-IR values were calculated. To determine fasting blood glucose levels of "prediabetes" and serum lipid levels of "dyslipidemia", individuals were divided into four groups: prediabetes (group 1, n=89), dyslipidemia (group 2, $\mathrm{n}=259$ ), prediabetes + dyslipidemia (group 3, $\mathrm{n}=71$ ) and control group (group 4, $\mathrm{n}=74$ ). The relationship between various parameters analyzed with SPSS 18.0 package program, $\mathrm{p}<0.05$ was considered significant.

Results: Mean Body Mass Index (BMI) was 39,4 $\pm 8,6 \mathrm{~kg} / \mathrm{m} 2$ and mean waist circumference was $108,8 \pm 16,0 \mathrm{~cm}$. Between prediabetes and control group BMI, waist circumference, fasting plasma glucose, triglycerides, total, HDL and LDL cholesterol levels were significantly different $(\mathrm{p}<0.05)$. A comparison between prediabetes + dyslipidemia group and control group showed significant differences in age, systolic and diastolic blood pressure, weight, BMI, waist and hip circumference, waist/hip ratio, fasting plasma glucose, $\mathrm{HbAlc}$, fasting insulin, HOMAIR, uric acid, ALT, triglycerides, total, HDL and LDL cholesterol levels $(\mathrm{p}<0.05)$.

Conclusion: It was concluded that obesity caused prediabetes and dyslipidemia. Among all parameters in studied groups, it was found that the most disorders was in prediabetes+dyslipidemia group. In conclusion, the coexistence of prediabetes and dyslipidemia in overweight and obese female patients increased the risk of cardiovascular more than other diseases.

\section{P20. Overexpression of myocardial connexin-43 due to} irradiation is suppressed by aspirin and atorvastatin

\section{Viczenczová $C^{\star}$, Szeiffová Bačová B, Kura B, Beňová T, Slezák J and} Tribulová $\mathbf{N}$

Institute for Heart Research, Slovak Academy of Sciences, Bratislava, Slovakia, E-mail: viczencz.csilla@gmail.com

Background: Radiotherapy is widely used in cancer treatment. However, it has been shown by our and other studies that radiation on the mediastinum is associated with cardiac disorders of connexin- 43 (Cx43). This intercellular channel protein is crucial for synchronized heart function. The aim of this study was to explore whether the treatment with aspirin, atorvastatin and sildenafil affect irradiationinduced alterations of cardiac Cx43 and PKC signaling.

Methods: Adult, male Wistar rats were divided into non-irradiated control rats and irradiated rats that were subjected to single dosage radiation on mediastinum at $25 \mathrm{~Gy}$. Both groups were treated with anti-inflammatory drug - aspirin (3 $\mathrm{mg} /$ day), hypolipidemic drug atorvastatin $(0.25 \mathrm{mg} /$ day $)$ and cardioprotective drug - sildenafil $(0.3$ $\mathrm{mg}$ /day) for six weeks and compared to untreated animals. Biometrical parameters were registered. Left and right ventricular tissues were taken for determination of total $\mathrm{Cx} 43$ expression and its functional phosphorylated forms (P-Cx43) using immunoblotting. Expression of PKC-epsilon, which directly phosphorylates $\mathrm{Cx} 43$ and pro-apoptotic PKC-delta was also determined.
Key results: Regardless the treatment, a significant decrease was found in body weight in irradiated rats. Neither heart nor left and right ventricles were affected due to irradiation and treatment. Irradiation induced the increased levels of total Cx43 expression in left and to lesser extent in right ventricles, also significantly enhanced $\mathrm{P}-\mathrm{Cx} 43$ in both ventricles. Aspirin and atorvastatin prevented elevation of total $\mathrm{Cx} 43$ as well as functional P-Cx43 in left ventricles only. Sildenafil enhanced total but not P-Cx43 in left ventricles and did not change total but suppressed $\mathrm{P}-\mathrm{Cx} 43$ in right ventricles of irradiated groups. Expression of PKC-epsilon was not affected by irradiation or treatment in left ventricles, while atorvastatin suppressed its levels in right ventricles of irradiated rats. Expression of PKC-delta was enhanced in rats after exposure to irradiation and treatment with aspirin and atorvastatin resulted in an additional increase.

Conclusion: Our results indicate that irradiation-induced upregulation of $\mathrm{Cx} 43$ in heartis prevented by treatment with aspirin and atorvastatin but not by sildenafil.

\section{P21. Omega-3 index associated with connexin 43: powerful indicator of malignant arrhythmias}

\section{Szeiffova Bacova $\mathbf{B}^{1^{*}}$, Viczenczova $C^{1}$, Benova $T^{1}$, Sec $P^{2}$, Certik $M^{3}$ and Tribulova $\mathrm{N}^{1}$}

${ }^{1}$ Institute for Heart Research, Slovak Academy of Sciences, Bratislava, Slovakia

${ }^{2}$ Institute of Biochemistry and Genetic of Animals, SAS, Bratislava, Slovakia

${ }^{3}$ Slovak University of Technology, Bratislava, Slovakia, E-mail: barbara.bacova@savba.sk

Background: We know from previous studies that combination of reduced $\mathrm{Cx} 43$ expression and increased collagen content (fibrosis) is associated with increase arrhythmogenicity. Low omega-3 ( $\omega-3)$ index was suggested as a risk factor for cardiovascular diseases and sudden cardiac death. We have previously shown that hypertensive rats benefit from $\omega-3$ fatty acid (FA) intake. Aim of this study was to explore relationship between $\omega-3$ index, expression of connexin 43 and incidence to ventricular fibrillation (VF) in aged male and female spontaneously hypertensive rats (SHR), a model of chronic pressure overload, hypertrophy and fibrosis.

Methods: One year-old SHR and age-matched healthy Wistar rats (WR) fed with $\omega$-3FA (Vesteralens, Norway, EPA+DHA 200mg/ day/2month) were compared with untreated rats. Gas chromatography was used for analysis of red blood cells (RBC) $\omega-3 \mathrm{FA}$ and $\omega-6 \mathrm{FA}$ composition. $\omega-3$ index was calculated as RBC level of eicosapentanoic acid (EPA) + docosahexanoic acid (DHA) expressed in percentage of total FA. Left ventricular tissue was taken especially for $\mathrm{Cx} 43$ and PKC-epsilon protein expression using Western blot method. Inducible ventricular fibrillation (VF) was examined using Langendorff-mode perfused heart.

Results: Comparing to healthy WR $\omega-3$ index was lower in both male and female SHR, i.e. $0.73 \%$ and $0.44 \%$ versus $1.75 \%$ and $1.17 \%$. This parameter was significantly increased due to $\omega-3$ FA intake to $2.38 \%$ and $3.34 \%$ in male and female SHR. Cx43 protein and its functional phosphorylated forms as well as corresponding PKCepsilon expression were decreased in both male and female SHR rats. Non-treated male and female SHR were much prone to inducible VF (100\% males and 65\% females) comparing to WR (65\% males and $35 \%$ females).This propensity, as well as expression of Cx43 and PKC- 
epsilon was significantly normalized in males and females SHR due to $\omega$-3FA intake.

Conclusions: These findings support the hypothesis that lower $\omega-3$ index associated with decreased expression of connexin 43 might be a powerful indicator of increased propensity of the heart to malignant arrhythmias.

P22. Cardioprotective and antiarrhythmic mechanisms of dietary fatty acids demonstrated in experimental model of hypertension

Tribulova $\mathrm{N}^{1^{*}}$, Viczenczova $\mathrm{C}^{1}$, Szeiffova Bacova $B^{1}$, Benova $T^{1}$, Knezl $\mathrm{V}^{2}$ and Slezak $\mathbf{J}^{1}$

${ }^{1}$ Institute for Heart Research, SAS, Bratislava, Slovakia,

${ }^{2}$ Institute of Experimental Pharmacology and Toxicology, SAS, Bratislava, Slovakia, E-mail:narcisa.tribulova@savba.sk

Background: Hypertension-related maladaptive myocardial remodeling, including abnormalities of $\mathrm{Cx} 43$, increases propensity of the heart to the life-threatening arrhythmias. Previous studies suggested that hypertension is accompanied by omega-3 fatty (omega3) acids deficiency and enhanced free radical production while there is a benefit of dietary oils intake. We aimed to prove our hypothesis that omega3 that affect cell membrane properties and antioxidant-rich red palm oil (RPO) may exert antiarrhythmic effects via modulation of myocardial electrical coupling protein $\mathrm{Cx} 43$. Design and Methods: Spontaneously hypertensive rats (SHR), which mimic essential hypertension in human, and age-matched normotensive rats were treated more than one month with highly purified omega3 $(200 \mathrm{mg} /$ day) or RPO (200mg/day) and compared with untreated rats. Biometrical parameters were registered and left ventricular tissue was taken for $\mathrm{Cx} 43$ analysis using immunolabeling, immunoblotting and real time PCR. Besides, the expression of PKC $\varepsilon$ and PKC $\delta$ were analyzed. Susceptibility of the heart to lethal ventricular fibrillation (VF) was examined in Langendorff-perfused heart. Key results: Both tested compounds reduced systolic blood pressure but did not affect heart or left ventricular mass. Hypertensive rat heart was much prone to VF compared to normotensive ones. In contrast, the threshold to induce VF was increased and VF incidence decreased upon treatment of hypertensive rats with omega3 or RPO. Antiarrhythmic effects were associated with increased expression of Cx43 mRNA and/ or $\mathrm{Cx} 43$ protein as well as enhanced its functional phosphorylated forms. Moreover, hypertension-related myocardial mislocalization (lateralization) of $\mathrm{Cx} 43$ was attenuated by treatments. Besides, an increase of PKCe that is implicated in $\mathrm{Cx} 43$ phosphorylation and decrease of $\mathrm{PKC} \delta$ that is involved in pro-hypertrophic signaling was detected. In conclusion, findings indicate that dietary oils can protect the heart from malignant arrhythmias, at least in part, by targeting myocardial $\mathrm{Cx} 43$ in experimental model of hypertension. Further studies should elucidate how the modulation of $\mathrm{Cx} 43$ affects myocardial electrical properties.

\section{P23. HPLC determination of trans-resveratrol and polydatin} in nutraceuticals

\section{Fibigr ${ }^{\star}$, Satinsky $D$ and Solich $P$}

Charles University in Prague, Faculty of Pharmacy, Department of Analytical Chemistry, Heyrovskeho 1203, 50005 Hradec Kralove, Czech Republic, E-mail: fibigrja@faf.cuni.cz

Trans-resveratrol and its glucoside polydatin are the naturally occurring antioxidant polyphenols. They occur in many plants, but grapes and related products are considered the most important dietary sources of these substances. The interest of these compounds began after the studies on the "French Paradox", in which cardioprotective effect of wine due to its polyphenolic composition, including resveratrol showed positive biological properties. The present contribution reports a novel HPLC method for quality control of resveratrol content in nutraceuticals on the Czech market. Nutraceuticals together with functional foods are among the most rapidly growing sectors in the food and personal care product industry. For these reasons a quality control is required and new modern analytical methodologies for quality control must be developed. Efficient separation of trans-resveratrol, polydatin and internal standard p-nitrophenol was achieved on the fused-core column Ascentis Express ES-CN (100 x $3.0 \mathrm{~mm})$, particle size $2.7 \mu \mathrm{m}$, with mobile phase acetonitrile/water solution of acetic acid $(0.5 \%) \mathrm{pH}$ $3(20: 80, \mathrm{v} / \mathrm{v})$ at a flow rate of $1.0 \mathrm{~mL}$ min- 1 and at temperature $60^{\circ} \mathrm{C}$. The detection wavelength was set at $305 \mathrm{~nm}$. Commercial samples of nutraceuticals were extracted with $100 \%$ methanol using ultrasound bath for $15 \mathrm{~min}$ and then filtered through $0.45 \mu \mathrm{m}$ PTFE filter. A $5-\mu \mathrm{L}$ of this sample solution was directly injected into the HPLC system. The developed method has shown high sample throughput during sample preparation process, modern separation approach and short time (3 $\mathrm{min}$ ) of analysis.

\section{P24. HPLC determination of indole-3-carbinol in food supplements}

\section{Fibigr $\mathrm{J}^{\star}$, Satinsky $\mathrm{D}$ and Solich $\mathbf{P}$}

Charles University in Prague, Faculty of Pharmacy, Department of Analytical Chemistry, Heyrovskeho 1203, 50005 Hradec Kralove, Czech Republic,E-mail: fibigrja@faf.cuni.cz

Indole-3-carbinol (I3C) is the breakdown product of naturally occurring glucosinolates, mainly in cruciferous vegetables such as broccoli, Brussels sprouts, cabbage and garden cress. In an acidic environment like the human stomach, $\mathrm{I} 3 \mathrm{C}$ is rapidly converted into an array of acid condensation products and modified derivatives. These indole derivatives have been shown to suppress the proliferation of various cancer cell lines, including those of breast, colon, prostate, and endometrium. The present contribution reports a novel HPLC method for quality control of indole-3-carbinol content in nutraceuticals on the Czech market. Food supplements together with functional foods are among the most rapidly growing sectors in the food and personal care product industry. Despite this fact no specific regulation exists in Europe to control food supplements, although they are considered under the same laws that regulate medicine and drug. For these reasons a quality control is required and new modern analytical methods for quality control must be developed. Efficient separation of Indole-3carbinol, internal standard ethylparaben, and other substances present in multicomponent nutraceuticals was achieved on core-shell column Kinetex $5 \mu$ XB-C18 100A $(100 \times 4.6 \mathrm{~mm})$, particle size $5.0 \mu \mathrm{m}$, with mobile phase acetonitrile/water according to the gradient program at a flow rate of $1.25 \mathrm{~mL}$ min- 1 and at temperature $50{ }^{\circ} \mathrm{C}$. The detection wavelength was set at $270 \mathrm{~nm}$. Commercial samples of nutraceuticals were extracted with $100 \%$ methanol using ultrasound bath for $10 \mathrm{~min}$ and an undissolved material was removed by centrifugation. A $5-\mu \mathrm{L}$ of the supernatant was directly injected into the HPLC system. The developed method has shown high sample throughput during sample preparation process, modern separation approach and short time (6.3 min) of analysis. 
P25. Association between Body Mass Index and total body fat on serum vitamin d status in sedentary group of croatian workers

Pavicic Zezelj $\mathrm{S}^{1,2^{*}}$, Kendel Jovanovic $\mathrm{G}^{1,2,}$ Mika F ${ }^{3}$, Crnkovic Milic $\mathrm{I}^{1}$, Cvijanovic $\mathrm{O}^{4}$, Linsak $\mathrm{Z}^{1}$ and Micovic $\mathrm{V}^{1,2}$

${ }^{1}$ Teaching Institute for Public Health of Primorsko-goranska County, Health Ecology Department, Rijeka, Croatia

${ }^{2}$ Faculty of Medicine, University of Rijeka, Department of Environmental Medicine, Rijeka, Croatia Italy

${ }^{3}$ RUOS Health and Occupational Medicine, Saipem s.p.a, Milan,

${ }^{4}$ Faculty of Medicine, University of Rijeka, Department of Anatomy, Rijeka,Croatia,E-mail: sandra.pavicic@zzjzpgz.hr

Vitamin D deficiency being associated to variety of chronic diseases and increased cardiovascular morbidity and mortality risk related to obesity is of great importance for health prevention especially of working population. The study aim was to assess the association of vitamin D status to obesity in sedentary group of Croatian workers. Study subjects were 105 healthy workers ( $46 \%$ men, $54 \%$ women) to which we assessed serum 25 -hydroxivitamin $\mathrm{D}(25(\mathrm{OH}) \mathrm{D})$ concentrations, body mass index (BMI), total body fat (TBF) percentage and dietary habits. The mean serum $25(\mathrm{OH}) \mathrm{D}$ concentration was $18.43 \pm 11.20 \mathrm{ng} / \mathrm{l}$. There were $67 \%$ workers with insufficient serum $25(\mathrm{OH}) \mathrm{D}$ less than $30 \mathrm{ng} / \mathrm{l}$ (ID) with mean $15.37 \pm 6.76 \mathrm{ng} / \mathrm{l}$ and mean serum $25(\mathrm{OH}) \mathrm{D}$ of $33 \%$ workers with adequate serum $25(\mathrm{OH}) \mathrm{D}(\mathrm{AD})$ was $36.79 \pm 4.39 \mathrm{ng} / \mathrm{l}$. We found that ID workers had higher BMI and TBF and their dietary vitamin $\mathrm{D}$ was lower compared to $\mathrm{AD}$ workers, but with no statistical significance. $\mathrm{AD}$ workers had slightly higher dietary intake of vitamin D sources than ID workers. Serum $25(\mathrm{OH}) \mathrm{D}$ was negatively and not significantly correlated to $\mathrm{BMI}(\mathrm{r}=-0.46 ; \mathrm{P}=0.727)$ and to TBF $(\mathrm{r}=-0.09$; $\mathrm{P}=0.474)$ and also we didn't observed significant difference among ID and $\mathrm{AD}$ workers groups between those correlations. With multiple linear regression analysis, $\mathrm{BMI}$ and $\mathrm{TBF}$ were found to have negative influence on serum 25(OH)D in ID more than in AD workers group as they had lower BMI and TBF values compared to ID workers group, but not statistically significant. Still, the study results are significant for public health obesity and cardiovascular prevention programs that should be more focused on promotion of Mediterranean dietary style, sources of vitamin D and physical activity.

P26. Taking marine fish may be a risk factor for insulin resistance in healthy koreans

\section{Neunghye $L^{\star}$}

Hwaseong Hyundaiajou Clinic, Korea, E-mail: idgwho@gmail.com

Chronic low-grade inflammation is a cause of insulin resistance. Serum mercury is related with inflammation. Many Koreans have marine fishes, and they contain mercury. This study investigated the association between serum mercury and insulin resistance. Subjects from the 2008-2010 Korean National Health and Nutrition Examination Survey (KNHANES) were selected $(n=29,235)$ and the relevant data of 5,388 subjects (2,643 males and 2,745 females) were analyzed cross-sectionally. Homeostasis model assessment for insulin resistance (HOMA-IR) was compared according to serum mercury quartiles and the odds ratio of having the highest quartile of HOMAIR according to serum mercury quartiles was calculated. Serum mercury levels in men and women were $5.90 \mu \mathrm{g} / \mathrm{L}$ and $4.12 \mu \mathrm{g} / \mathrm{L}$, respectively, and fasting blood sugar (FBS), insulin, and HOMA-IR were significantly correlated with serum mercury. The correlation was stronger in men than in women. In men, FBS and HOMA-IR showed step-wise increases as the quartiles of serum mercury increased. HOMA-IR was significantly different in the third and fourth quartiles of serum mercury compared with the first quartile. In women, FBS and HOMA-IR were significantly different in the third and fourth quartiles of serum mercury compared with the first quartile. In addition, the odds ratio of having the highest quartile of HOMA-IR was significant in the top quartile of serum mercury (OR=1.720, 95\% CI; 1.172-2.526) compared with the lowest quartile in men. In this large populationbased study, serum mercury was correlated with HOMA-IR and may be a risk factor for insulin resistance in the Korean population.

\section{P27. Reduction of body weight by dietary ginger is associated} with mitochondrial biogenesis

\section{Jung $S^{\star}$, Oh S, Lee MS, Kim S and Kim Y}

Department of Nutritional Science and Food Management, Ewha Womans University, Seoul, Republic of Korea,E-mail: cococosy@naver. com

Obesity comes from a chronic disruption in balance between energy intake and energy expenditure. Impaired mitochondrial function and dyslipidemia in obesity are some of characteristics resulting in obesitylinked metabolic disorders. This study investigated anti-obesity effect of ginger extract in high-fat diet fed rats. Specifically, we focused on its ability to modify mitochondrial biogenesis and lipid profiles in this study. Male, Sprague-Dawley rats were randomly divided into three groups and fed each experimental diets for 14 weeks: HF, 45\% highfat diet fed group; GEL, $45 \%$ high-fat diet $+0.25 \%$ ginger extract fed group; GEH, $45 \%$ high-fat diet $+0.5 \%$ ginger extract fed group. At the end of the experiment, the final body weight and white adipose tissue mass were decreased by the ginger supplementation. The food intake and energy efficiency ratio did not differ among the three groups, which implies ginseng extract had no anorexic effect. Increased mitochondrial size and number in muscle and brown adipose tissue was shown by the dietary ginger. Mitochondrial DNA contents and mRNA levels of peroxisome proliferator-activated receptor $\gamma$ coactivator-1 $\alpha($ PGC-1 $\alpha$ ), nuclear respiratory factor-1(NRF-1) and transcription factor $\mathrm{A}(\mathrm{Tfam})$ in skeletal muscle were increased in the ginger fed groups, which suggests enhanced mitochondrial biogenesis. Furthermore, ginger extract elevated the levels of HDL-cholesterol in serum and liver compared to the HF control group. Upregulated mRNA expression of genes including ATP-binding cassette transporter A1(ABCA1), Apolipoprotein A-1(ApoA-1) and lecithin:cholesterol acyltransferase(LCAT) in the liver might also explain the effects of dietary ginger on HDL-cholesterol metabolism. These results indicate that dietary ginger extract might have beneficial effect on obesity by enhancing muscle mitochondrial function and HDL-cholesterol metabolism.

\section{P28. Comparison between calcium intake and excretion of Korean adult by age}

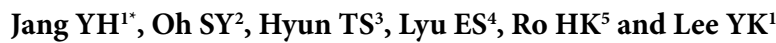

${ }^{1}$ Department of Food Science and Nutrition, Kyungpook National University, Daegu, Korea

${ }^{2}$ Department of Food Science and Nutrition, Kyunghee University, Seoul, Korea 
${ }^{3}$ Department of Food and Nutrition, Chungbuk National University, Cheongju, Korea

${ }^{4}$ Department of Food Science and Nutrition, Pukyong National University, Busan, Korea

${ }^{5}$ Department of Food and Nutrition, Dongshin University, Naju, Korea, E-mail: missjang90@naver.com

In comparison to the fact that the amount of recommended nutrient calcium intake based on the Dietary Reference Intakes for Korean adults is approximately $700 \mathrm{mg}$, the amount of average calcium intake of Korean adults per day is $511 \mathrm{mg}$, which is rather low. The purpose of this study is to evaluate the calcium intake status of Korean through the analysis of calcium intake and excretion targeting Korean adults. The subjects of this study were 640 healthy Korean adults aged 20 to 69 who lived in 8 cities within 4 regions including 320 males and 320 females. The amount of calcium intake was investigated using 24hour recall method for twice, and the amount of calcium excretion and the urine creatinine concentration were analyzed through 24-hour urine collection for twice. The average amount of calcium intake was $549.1 \mathrm{mg}$ (306 mg for animal calcium, $243 \mathrm{mg}$ for vegetable calcium), which was no significant difference between ages. The average amount of calcium intake per $1,000 \mathrm{kcal}$ was $289.3 \mathrm{mg}$ and 60 s showed the highest amount and 20 s showed the lowest $(\mathrm{p}<0.001)$. The average amount of calcium excretion was $0.2 \mathrm{~g} / \mathrm{d}$ and 20 s showed the lowest $(\mathrm{p}<0.05)$. And, 20s showed the highest blood calcium content $(\mathrm{p}<0.05)$. The urine calcium-creatinine ratio $(\mathrm{Ca} / \mathrm{Cr})$ was 0.16 and females showed a higher ratio than males in all ages, and all subjects are within the normal range. The quintile analysis result of calcium excretion showed that BMI $(\mathrm{p}<0.001)$, energy intake $(\mathrm{p}<0.05)$ and protein intake $(\mathrm{p}<0.05)$ was highest at the top quintile of calcium excretion. The results above showed that the amount of Korean adult's calcium intake was approximately $78.4 \%$ of the recommended nutrient intake, indicating that the nutritional education for increasing calcium intake would be necessary for Korean adults because the intake amount of calcium is still insufficient.

P29. LC-MS analysis, antioxidant and antimicrobial activities for hyssopus officinalis and ocimum basilicum from Romania

\section{Tiperciuc $B^{\star}$, Vlase L, Hanganu D and Benedec D}

Iuliu Hatieganu University of Medicine and Pharmacy, 13, E. Isac Street, 400023 Cluj-Napoca, Romania,E-mail: brandu32@yahoo.com

The aim of this work was to study the chemical composition, antioxidant and antibacterial properties of two Romanian species: Hyssopus officinalis L. and Ocimum basilicum L. Qualitative and quantitative analysis of the major phenolic compounds were conducted using high-performance liquid chromatography coupled to mass spectrometry (HPLC-MS). The total phenolic and flavonoidic content was spectrophotometrically determined. The extracts were screened for antioxidant activities using four in vitro assay models: DPPH radical scavenging assay, ferric reducing ability plasma (FRAP) method, cupric reducing antioxidant capacity (CUPRAC) assay, and an electron paramagnetic resonance (EPR) radicals' detection. The antimicrobial tests were performed using the disk diffusion assay. For Ocimum basilicum extract, the phenolic acid profile showed the presence of phenolic acids, and flavonoids (rutin, quercetin, kaempferol). In the aerial parts extract of hyssop the hydroxycinnamic acid derivatives (caftaric, caffeic acids) and the aglycones of flavone (luteolin, apigenin) were detected. The highest amount of the total polyphenols and flavonoids was determined in the extract of the basil.
The results showed that $\mathrm{O}$. basilicum extract was more antioxidant than H. officinalis extract, related with thepolyphenolic total content. The antimicrobial tests underlined an important activity against Staphylococcus aureus and Candida albicans for these samples. The phytochemical and pharmacological potential shown in this study will help to maximize the desired therapeutic benefits of these well known medicinal plants.

P30. Consumption of protease preparations derived from aspergillus markedly elevates cecal and organic acids in rats fed a high-fat diet

\section{Iwamoto $A^{\star}$, Yang $\mathrm{Y}$ and Kato $\mathrm{N}$}

Graduate School of Biosphere Science, Hiroshima University, E-mail:M152853@hiroshima-u.ac.jp

Recent our study has indicated that consumption of Aspergillus awamori-fermented burdock caused a marked elevation in cecal Bifidobacterium in rats fed a high-fat diet when compared with that of unfermented-burdock. Our preliminary study further showed consumption of two protease preparations derived from Aspergillus among several protease preparations commercially available also markedly elevated cecal Bifidobacterium. This study investigated the effects of dietary addition of the two proteases preparations derived from Aspergillus on the colonic luminal environment. Rats were fed $30 \%$ beef tallow diet with or without the protease preparations including Amano protease (Protease A "Amano SD," neutral proteases from Aspergillus spp.) or Orientase (Orientase $\mathrm{AY}$, acid proteases from Aspergillus niger) at the dose of $0.2 \%$ for 3 weeks. Cecal Bifidobacterium was dramatically elevated in the dietary Amano protease group (194 fold, $\mathrm{p}<0.05)$ but not the Orientase group. Lactobacillus was elevated in both groups $(\mathrm{p}<0.05)$. Cecal n-butyrate, propionate, and lactate were higher in the Amano protease and Orientase groups than the controls $(\mathrm{p}<0.05)$. Fecal IgA and mucins were elevated in the Amano protease group ( $\mathrm{p}<$ 0.05 ). These results suggest the potential useful effect of consumption of Aspergillus-derived protease preparations favorable for the colonic luminal environment in rats fed a high-fat diet.

\section{P31. Nutrition as a risk factor for changes in health status}

Semenova $\mathrm{VN}^{\star}$, Krasheninina GI, Galuzo NA, Fedyanina NS, Kozupeeva DA, Porotikova AY, Haritonova TA, Xannanoeva AM, Goman AV, Kochneva and Averyanova TA

Novosibirsk State Medical University, Novosibirsk, Russia, E-mail: tezis-ngmu@mail.ru

It is believed that food is the basis or essential in the emergence, development and approximately $80 \%$ of all known pathological conditions. In addition to significant contribution to the formation, preservation and strengthening of health of the population, food has its inherent feature high-quality control at the individual, family, group level.

The design of the study: This paper presents the results of studying the role of nutrition as a risk factor for the onset and exacerbation of diseases of the gastrointestinal tract, hepatobiliary system and kidneys; diseases, current medical, social and organizational points of view. A significant number of these patients are of working age, which explains the high relevance of the search for new methods of treatment and prevention of relapse of these pathological States. The object of the study was the patients of the clinics. Some medical records and food diaries 
have been analyzed, as well as sociological research (questionnaires, supplemented by interviews).

Results: Analysis of food diaries 150 ESRD patients undergoing dialysis showed that only one fifth of patients adhere to proper diet. In this group of patients revealed no clinically significant complaints, biochemical parameters were within normal limits. The main changes from "violators" of the diet was to increase body mass (in 50\% increase in interdialytic period was $3.5 \mathrm{~kg}, 30 \%$ had reached $4.5 \mathrm{~kg}$ instead of "laid" $2-3 \mathrm{~kg}$ ) of urea and creatinine in the serum 2 times (respectively $35,0 \pm 1,82$ against $18,5 \pm 0,91, \mathrm{~mol} / \mathrm{l}, \mathrm{p}=0.001 ; 1315,0 \pm 80,27$ against $628,5 \pm 44,18 \mathrm{~mol} / \mathrm{l}, \mathrm{p}<0.001)$. Analysis of complaints and the clinical and biochemical parameters allowed us to separate the observed "offenders" diet for 4 groups, highlighting three major complications (arterial hypertension, hypercalcemia, BEN) and the so-called mixed group (combining the complaints of all these groups and is characterized by variability of biochemical parameters). It is shown that the major causes of cancer of the colon, most patients are improper and irregular meals ( $83 \%$ vs. $21 \%$ in the control group) and concomitant diseases of the gastrointestinal tract (in 85.7\%; least, gastritis, pancreatitis, stomach ulcers, hemorrhoids). Unnecessary calories in the presence of physical inactivity at $76 \%$, followed by obesity ( $80 \%$ of patients), also increases the risk of colon cancer. 96 patients with diagnosis of chronic cholecystitis on the basis of the analysis were divided into 4 groups. Unhealthy lifestyle $45,8 \%$ leads in $61 \%$ of cases exacerbate, $71 \%$ have hidden cholelithiasis and half (53\%) of this group cholecystitis becomes acute, and in $12.5 \%$ respectful of correct eating behavior and rational motive mode, respectively, $-36 \% ; 51 \%$ and $17 \%$.

Conclusion: The research shows the importance of nutrition, especially nutrition as a risk factor for the development and exacerbation of diseases. Research findings demonstrate the necessity of hygiene education and awareness of the population, not only for formation of a healthy lifestyle and eating habits but also for early diagnosis and appropriateness of preventive examinations.

\section{P32. Effect of low dose of alcohol in Kkay obese/diabetic mice}

Takahara $\mathrm{K}^{1^{*}}$, Kimoto $\mathrm{A}^{1}$, Kato $\mathrm{N}^{1}$ and $\mathrm{Izu}^{2}$

${ }^{1}$ Hiroshima University, Higashi-Hiroshima 739-8528, Japan

${ }^{2}$ National Research Institute of Brewing, Higashi-Hiroshima 7390046, Japan, E-mail: M156879@hiroshima-u.ac.jp

Accumulating epidemiological evidence suggests beneficial effect of low dose of alcohol on the development of diseases including cardiovascular disease, diabetes, liver cirrhosis, cancers, etc. However, there are very limited animal studies on the effect of low dose of alcohol. Recently, we have reported that low dose of ethanol improves liver function in rats fed a high-fat diet (1). Furthermore, our studies with senescence-accelerated prone-mice 1 and 2 (SAMP1 and SAMP8) have suggested that low dose of ethanol retards the aging. This study was aimed to elucidate the effect of low dose of ethanol in KKAy obese/ diabetic mice. Male KKAy mice (6 week old) were fed a commercial stock diet for 10 weeks. Ethanol was given with drinking water containing $1 \%$ and $2 \%(\mathrm{v} / \mathrm{v})$ ethanol. The body weight, liver weight, skeletal muscle weight and adipose tissue weight were unaffected by intake of ethanol. One \% ethanol intake caused a significant reduction in serum total cholesterol $(\mathrm{P}<0.05)$, but $2 \%$ ethanol intake did not. Serum levels of glucose, triglyceride, insulin and leptin were unaffected. Intraperitoneal glucose-tolerance test indicated no influence of ethanol intake on the glucose tolerance. Interestingly, serum level of adiponection was lower in the $1 \%$ and $2 \%$ ethanol groups $(\mathrm{P}<0.05)$.
Serum levels of inflammatory cytokines were unaffected. Our study provides evidence for the beneficial effect of low dose of alcohol on blood cholesterol and adiponectin in KKAy mice.

P33. Protective effects of lactobacillus plantarum hy7714 against uvb-induced wrinkle formation and skin dryness in hairless mice

Jeung $\mathrm{W}^{1^{*}}$, Kim $\mathrm{S}^{1}$, Jeong $\mathrm{J}^{1}$, Choi $\mathrm{I}^{1}$, Huh $\mathrm{C}^{2}$, Sim $\mathrm{J}^{1}$, Ahn $\mathrm{Y}^{1}$

${ }^{1}$ R\&BD Korea Yakult Co., Ltd., Yongin 446-901, Republic of Korea

${ }^{2}$ Institute of Green Bio Science and Technology, Graduate School of International Agricultural Technology, Seoul National University, Pyeongchang 232-916, Republic of Korea, E-mail: wjeung@re.yakult. co.kr

Ultraviolet (UV) irradiation causes alterations in skin collagenous tissues caused by the breakdown of collagen, a major structural protein in the dermis. Collagen destruction by chronic sun exposure is one of the key factors contributing to the aged appearance of the skin. A large number of studies regarding UV-induced molecular mechanisms and signal transduction have been reported. The overriding factor in collagen loss is the activation of the collagenase matrix metalloproteinase (MMP)-1 by UVB exposure. MMPs are a family of zinc-dependent endopeptidases expressed in several cell types that degrade macromolecules from the extracellular matrix (ECM). Among these enzymes, MMP-1 plays an important role in the degradation of native types I and III collagen in human skin. Once cleaved by MMP1, collagen can be further broken down into small peptides by MMP9, resulting in ECM degradation. Reduction in collagen fibers is an important mechanism involved in the development of skin aging. In recent years, probiotics have gained interest due to their beneficial effects on skin health, such as inhibiting atopic dermatitis and improving skin immunity or inflammation. In addition to protecting against cutaneous photo damage, it has been suggested that a specific strain of lactic acid bacteria showed anti-aging effect such as reduction in wrinkle formation and improved elasticity in hairless mice. In the present study, we studied the protective effects of Lactobacillus plantarum HY7714 against UVB-induced wrinkle formation and skin dryness in hairless mice. First, we evaluated anti-wrinkle effect of L. plantarum HY7714 in UVB-irradiated human dermal fibroblast (Hs68) cells and hairless mice. Results showed that L. plantarumHY7714 treatment effectively rescued UVB-reduced procollagen expression through the inhibition of UVB-induced MMP-1 expression in Hs68 cells. Western blot showed that L. plantarumHY7714 inhibited the phosphorylation of Jun N-terminal kinase, thereby suppressing the UVB-induced phosphorylation and expression of c-Jun. Oral administration of L. plantarumHY7714 at a dose of $1 \times 109$ CFU/ day to hairless mice exposed to UVB radiation for 8 weeks clearly inhibited the number, depth, and area of wrinkles in hairless mouse skin. Histological data showed that L. plantarumHY7714 significantly inhibited UVB-induced epidermal thickness in mice. Western blot and zymography data also revealed that L. plantarumHY7714 effectively inhibited MMP-13 expression as well as MMP-2 and -9 activities in dermal tissue. Second, we evaluated the effect of L. plantarumHY7714 on skin hydration in Hs68 cells and hairless mice. L. plantarumHY7714 not only increased the serine palmitoyltransferase (SPT) mRNA level, but also decreased the ceramidase mRNA level. In order to confirm the skin hydrating effect of L. plantarumHY7714 in vivo, we orally administered L. plantarumHY7714 at a dose of $1 \times 109 \mathrm{CFU} /$ day to hairless mice exposed to UVB radiation for 8 weeks. Oral administration of L. plantarumHY7714 not only decreased UVB-induced epidermal 
thickness, but also suppressed the increase in transepidermal water loss (TEWL) and decrease in skin hydration, which reflects barrier function fluctuations following UV irradiation. In particular, L. plantarumHY7714 administration increased the ceramide level compared with that in the UVB group. In the experiment on SPT and ceramidase mRNA expressions, L. plantarumHY7714 administration improved the reduction in SPT mRNA levels and suppressed the increase in ceramidase mRNA levels caused by UVB in hairless mice.

Collectively, we determined the UV-protective effects of $\mathrm{L}$. plantarum HY7714, which decreases UVB-induced epidermal thickness, wrinkle formation, skin hydration loss, and TEWL, as observed in the UVB-irradiated hairless mice model. Thus these results suggest that L. plantarumHY7714 can be a potential candidate for anti-photoaging agent and preserving skin hydration levels against UV irradiation.

P34. Phlorotannin-rich fraction from ishige foliacea brown seaweed ameliorate the scopolamine-induced memory impairment in mice

\section{Um $\mathrm{M}^{*}$, Son H, Kim Y, Cho S, Kim I, Han D and Lee C}

Divisions of Functional Food Research, Korea Food Research Institute, Seongnam 463-746, Republic of Korea, E-mail: myum@kfri. re.kr

Ishige foliacea is a species of brown algae on the coasts of Korea and Japan and has shown to contain specific polyphenols knowns as phlorotannins. The phlorotannins are reported to have antioxidant, anticancer, anti-diabetic, and anti-inflammatory functions. Taking those facts into account, we hypothesized that phlorotanninrich fraction from Ishige foliacea brown seaweed (PRFI) exerts a neuroprotective effect. To investigate whether PRFI can preserve cognitive function, we examined its effects on scopolamine-induced learning and memory impairment in mice using the Morris water maze test and passive avoidance test. PRFI ( 50 or $100 \mathrm{mg} / \mathrm{kg}$ ) was administered to ICR mice by oral gavage for 6 weeks, and memory impairment was induced by intraperitoneal injection of scopolamine $(1 \mathrm{mg} / \mathrm{kg})$. As a result, PRFI supplementation restored the escape latency and number of crossing to near normal levels in Morris water maze test. No significant differences were found in swim speed or in the escape latency among the experimental groups during the course of four consecutive training trials. In addition, it exerted longer stepthrough latency than those of scopolamine-treated group in passive avoidance test. Supplementation with PRFI significantly decreased the activity of acetylcholinesterase in brain of the scopolamine-treated mice, as well as lipid peroxidation and oxidized gluthathione levels. Accordingly, expression of brain-derived neurotrophic factor and phosphorylation of extracellular signal-regulated kinases $1 / 2$ and cAMP response element-binding protein involved neuroplasticity in the hippocampus of PRFI groups were higher than those of the scopolamine-treated group. Collectively, these findings suggest that PRFI may prevent memory impairment, in part through the regulation of neuroplasticity signaling pathway.

P35. Isolation of bacteriocin (enterocin) producer enterococci strains from different type cheese and identification of bacteriocins by polymerase chain reaction (pcr)

\section{Özden Tuncer $B^{\star}$ and Avcı M}

Faculty of Engineering, Department of Food Engineering, Süleyman Demirel University, Isparta, Turkey, E-mail: banutuncer@sdu.edu.tr
Lactic acid bacteria (LAB) is a diverse group of beneficial bacteria which have been in advertently used by mankind for thousounds of year. Enterococci are the dominant lactic acid bacteria (LAB) in many foods, including vegetables, meat and dairy products. Many studies indicate that these bacteria play a major role in the development of the sensory characteristics of fermented foods such as traditional cheeses, sausages and olives. The ability of enterococci to produce antimicrobial peptides known as bacteriocins that could be used as food/feed biopreservatives is also remarkable. The bacteriocins (enterocins) produced by enterococcal strains include the commonly encountered enterocins A, B, P, AS-48, L50A, L50B, 1071A, 1071B and Q. Due to the biotechnological potential of enterococci in food and health, the purpose of this study is to determine the antimicrobial activity and the occurrence of bacteriocin structural genes in Enterococcus spp. isolated from different cheeses. A total of 100 presumptive Enterococcus strains were isolated from 33 different cheese samples on KAA medium and grown in MRS broth. All isolates were Gram-positive, catalasenegative and grown at $10{ }^{\circ} \mathrm{C}, 37^{\circ} \mathrm{C}$ and $45^{\circ} \mathrm{C}$, in the presence of $\mathrm{NaCl}(6.5 \%)$ and at $\mathrm{pH} 9.6$ in Elliker broth medium. In addition all of them were found resistant to heat at $60{ }^{\circ} \mathrm{C}$ for 30 and $60 \mathrm{~min}$. The 11 presumptive Enterococcus isolates showed ihbition zones against to different indicator microorganisms. Proteinase $\mathrm{K}$ treatment showed that antimicrobial substances of 11 isolates have proteinoeus nature. The 11 enterocin producer isolates were identified genotypically by $16 \mathrm{~S}$ rDNA homology and determined as E. faecalis (5) and E. faecium (6) with the similarity percentage of $93-99 \%$ when 16 S rDNA PCR amplicons compared to GenBank. Enterocin genes were detected with using most common enterocin primers. The entA gene was detected in 7 strains while 8 strains carry ent $X$ gene. The ent $B$ and entP genes were detected in 4 and 2 strains, respectively. The genes coding for enterocin L50A/B, bacteriocin 31, enterocin AS-48, enterocin Q,enterocin 1071 and enterocin cylLL/S were not detected in any Enterococcus strains. In the present study enterocins were identified using known enterocin primers and multiple enterocin genes were detected in 7 strains. Two unidentified enterocins should be investigated whether the new bacteriocins.

P36. Detection of enterotoxin genes in coagulase-negative staphylococcus and macrococcus caseolyticus strains from turkish dry fermented sausage (sucuk)

\section{Tuncer $\mathrm{Y}^{\star}$ and Çetin $\mathrm{H}$}

Faculty of Engineering, Department of Food Engineering, Süleyman Demirel University, Isparta, Turkey,E-mail: yasintuncer@sdu.edu.tr

Gram-positive, catalase-positive cocci (especially coagulasenegative Staphylococci) are known to be technologically important in the fermentation of dry fermented sausages. Sucuk is a popular dry fermented sausage in Turkey. In addition, it is popular not only in Turkey but also in many Middle Asian, Middle Eastern, SouthEastern and Northern European countries. Although sucuk is one of the most popular traditional meat products in Turkey, there is not enough data on the enterotoxigenic potential of its coagulase-negative staphylococcal microflora. The purpose of this study was to determine the enterotoxin-encoding genes in coagulase-negative Staphylococcus and Macrococcus caseolyticus strains isolated from sucuk, produced by different manufacturers without using starter culture. A total of 51 coagulase-negative Staphylococcus and $10 \mathrm{M}$. caseolyticus strains were tested for the presence of 18 enterotoxin genes; sea, seb, sec, sed, see, seg, seh, sei, selj, selk, sell, selm, seln, selo, selp, selq, selr and tst1 by the multiplex-PCR. All coagulase-negative Staphylococcus and M. 
caseolyticus strains were negative to all enterotoxin genes. The results of this study showed that absence of risk related to the enterotoxogenic potential of coagulase-negative Staphylococcus and M. caseolyticus strains as indigenous microbiota from traditional Turkish dry fermented sausage (sucuk).

\section{P37. Vitamin d decreases adipocyte lipid storage and increases lipolysis and sirt1 expression in 3t3-11 adipocytes}

\section{Chang $E^{\star}$ and Kim $Y$}

Department of Nutritional Science and Food Management, Ewha Womans University, Seoul, Korea, E-mail: eugenics77@hotmail.com

Rapidly increased prevalence of obesity becomes worldwide epidemic due to its contribution to chronic diseases including type 2 diabetes, cardiovascular disease and nonalcoholic fatty liver disease. Evidence is accumulating to show that low vitamin D status is closely associated with increased body fat mass and obesity; however the mechanisms by which vitamin D has beneficial effects have not been fully determined. This study was designed to investigate the influence of 1,25 dihydroxyvitamin $\mathrm{D}(1,25(\mathrm{OH}) 2 \mathrm{D})$ on lipid storage and metabolism in adipocytes. Differentiated 3T3-L1 adipocytes were treated with $1,25(\mathrm{OH}) 2 \mathrm{D}(100 \mathrm{nM})$ for 24 hours. MTT cell proliferation assay was employed to measure effect of $1,25(\mathrm{OH}) 2 \mathrm{D}$ on cell viability. The intracellular lipid content was evaluated by Oil Red O staining. Glycerol level in culture medium was measured to demonstrate effect of $1,25(\mathrm{OH}) 2 \mathrm{D}$ on lipolysis. RT-PCR was performed to determine adipogenesis-related markers such as aP2, C/EBPa, PPAR $\gamma$, and SCD1, fatty acid oxidation-associated factors including CPT1a, PGC1 $\alpha$, and PPARa, and sirtulin1 (SIRT1) expression, a nicotinamide adenine dinucleotide (NAD)-dependent deacetlyase involved in apoptosis, energy homeostasis, and longevity. 24 hour incubation of $1,25(\mathrm{OH}) 2 \mathrm{D}$ $(0,1,10,100 \mathrm{nM})$ did not alter cell viability. $1,25(\mathrm{OH}) 2 \mathrm{D}$ treatment (100 nM, 24 hour) significantly induced a reduction in intracellular lipid content and an increase in basal and isoproterenol-induced lipolysis. 1,25( $\mathrm{OH}) 2 \mathrm{D}$ significantly decreased gene expression of aP2, $\mathrm{C} / \mathrm{EBP} \alpha, \mathrm{PPAR} \gamma$, and SCD1. In contrast, mRNA levels of CPT1 $\alpha$, PGC1 $\alpha$, and PPAR $\alpha$ were significantly up-regulated by $1,25(\mathrm{OH}) 2 \mathrm{D}$ treatment. Moreover, SIRT1 expression was significantly increased by $1,25(\mathrm{OH}) 2 \mathrm{D}$ incubation. These findings suggest that $1,25(\mathrm{OH}) 2 \mathrm{D}$ might decrease adipocyte triglyceride storage and increase lipolysis and SIRT1 expression in 3T3-L1 adipocytes.

\section{P38. The metabolic and morphological changes in hepg2 cells treated with resorcinolic lipids and ethyl alcohol}

\section{Piwoni $A^{\star}$, Rożalska A and Stasiuk M}

University of Wroclaw, Faculty of Biotechnology, Laboratory of Lipids and Liposomes, Fryderyka Joliot-Curie 14a, 50-383 Wroclaw, Poland, E-mail: adriana.piwoni@gmail.com

High dietary intake of whole grain cereal is associated with decreasing risk of many diet-related diseases including cardiovascular disease, type 2 diabetes and some types of cancer. The whole grainoriginating substances responsible for these protective effects are still not identified. Alk(en)ylresorcinols are proposed to be one of them. Since ARs occur only in the outer layer of the grain, it has been proposed recently that the ARs content can be recognized and used as a biomarker for whole grain rye and wheat product's intake [3]. ARs has been shown as a very promising compounds having antioxidant, antigenotoxic, cytostatic and antibacterial activity.
The aim of this project was verification of ARs (5-n-alk(en) ylresorcinols, resorcinolic lipids) ability to protect HepG2 cells (human liver hepatocellular carcinoma cell line) against the effects of excess supply of ethanol - compound with previously proven a hepatotoxic activity. Obtain results shown that ARs when added to the culture medium increased the number of surviving cells in a manner dependent on incubation time and concentration of ARs in the medium. The incubation of HepG2 cells with resorcinolic lipids and then with ethanol eliminates the toxic effect of ethanol alone on the tested cells. We examined also the physiological and morphological state of cells after treatment with ethanol and ARs (alone and together). Indicators of physiological state of the cell were e.g. a lactate dehydrogenase (LDH) leakage assay, accumulation of intracellular ROS (Reactive Oxygen Species) and measurement of lipids peroxidation. The morphological changes were visualized using fluorescence microscopy.

\section{P39. Proper diet extending the healthy life expectancy of japanese people in a post-aged society}

\section{Nakagawa $Y^{\star}$, Karashima J, Hasegawa $M$ and Yamada $S$}

Jissen Women’s University, Japan, E-mail: nakagawa-yasue@jissen. ac.jp

"Healthy eating" importantly promotes Japanese people's longevity throughthe three stages of human life children in the fast stages of life.In the second stage of life, adults inhibit health deterioration, and prevent the onset of lifestyle diseases, by to be able to continuing an appropriate diet.In the third stage of life, elderly people prevent age-relatedfrailty and maintain their quality of life, by continuing toimprove eating habits in light of past experience. In order to support this hypothesis, an intervention was carried out, in which elderly peopleexaminedtheir own eating habits using commercially available ingredients. This study aimed to obtain specific information about diets that are healthy for elderly people. Participants were 3 men and 51 women totaling 54 individuals.The men's average agewas 72.3 years, that of women was 73.8 years.Participantshad been introduced to commercial diet ingredients from four to nine months previously.Interviews were conducted after the intervention. Obtained interview data described intervention materials, dietary changes after intervention, and participants' ongoingdiet progress. After the intervention, 57\% of participants expressed that their diet had improved, $41 \%$ expressed observing no change, and $2 \%$ did not give valid responses. Participants expressed many reasons for absence ofimprovement, including longstandinghabit, and not recognizing a need for dietaryimprovement to promote health.Regarding food preparation habits after the intervention, $44 \%$ of participants cooked at home, a $31 \%$ increase from before the intervention. Eighteen percent of participants indicated preferring homecooked food.Regarding ongoingsupport, improvement of simple cooking techniques, such as regularly providing publicfood education, may facilitate ongoing dietaryimprovement in society, this may it has been suggested to 1 extend the healthy lifespan elderly people.Educatingelderly people about meal ingredientssupporthealthyeating habitsamong elderly people.

P40. Microbiological quality of ice and ice machines used in food industry

\section{Bingol EB ${ }^{1 *}$, Hampikyan $\mathrm{H}^{2}$, Colak $\mathrm{H}^{2}$ and Cetin $\mathrm{O}^{1}$}

${ }^{1}$ Department of Food Hygiene and Technology, Faculty of Veterinary Medicine, Istanbul University,34320, Istanbul, Turkey 
${ }^{2}$ Department of Gastronomy and Culinary Arts, The School of Applied Sciences,Beykent University, 34500, Istanbul, Turkey, E-mail: bingolb@istanbul.edu.tr

The ice used in food industry has to be safe and the water used in ice production should have the quality of drinking water. The consumption of contaminated ice directly or indirectly may be a vehicle for transmission of pathogenic bacteria to human yielded with outbreaks of gastrointestinal diseases. The objective of this study is to monitor the microbiological quality of ice, the water used in producing ice and the hygienic conditions of ice making machines in various food enterprises. E. coli was detected in 7 (6.7\%) ice and 23 (21.9\%) ice chest samples whereas E. coli was negative in all examined water samples. Psychrophilic bacteria were detected in 83 (79.0\%) out of 105 ice chest and in $68(64.7 \%)$ out of 105 ice samples, whereas enterococci were detected only in $13(12.4 \%)$ ice samples. Coliforms were detected in $13(12.4 \%)$ water, $71(67.6 \%)$ ice chest and 54 (51.4\%) ice samples. In order to improve the microbiological quality of ice, the maintenance, cleaning and disinfecting of ice machines should be carried out effectively and periodically. Meantime, high quality water should be used for ice production.

\section{P41. The polycyclic aromatic hydrocarbons (pahs) occurence in döner kebap cooked under different heating sources}

\section{Colak $\mathrm{H}^{1^{*}}$, Hampikyan $\mathrm{H}^{2}$, Bingol $\mathrm{EB}^{1}$, Cetin $\mathrm{O}^{1}$ and Bingol $\mathrm{B}^{3}$}

${ }^{1}$ Department of Food Hygiene and Technology, Faculty of Veterinary Medicine, Istanbul University, 34320, Istanbul, Turkey

${ }^{2}$ Department of Gastronomy and Culinary Arts, The School of Applied Sciences, Beykent University, 34500, Istanbul, Turkey

${ }^{3}$ Nobel Pharmaceutical, Inkilap Mah., Akcakoca Sok., No. 10, Umraniye, 34768, Istanbul, Turkey, E-mail: hcolak@istanbul.edu.tr

Döner kebab is a traditional Turkish meat product produced from lamb, veal, beef or poultry meat and then seasoned with salt, pepper, onions, cumin, allspice and thyme. The intact or ground muscle and some animal fats $(20-40 \%)$ are shredded and then mixed with seasoning materials and melded to give a cone like shape and impaled on a döner kabab stick. The raw döner is slowly rotated in a vertical position in front of a heating source such as open gas, electric oven or charcoal to roast the surface. During the thermal processes some harmful compounds such as polycyclic aromatic hydrocarbons (PAHs) may be occurred on the surface of döner kebabs. The aim of this study is to determine $16 \mathrm{EU}$ priority PAHs in 200 meat döner kebab samples which are produced under four different heating sources obtained randomly from various buffets and restaurants located in Istanbul. The samples were analyzed by means of GC-MS. According to results all analyzed PAH compounds were detected in different levels and benzo[a]pyrene which is a good marker for total PAHs in foods, ranged between $0.29-32.41 \mu \mathrm{g} / \mathrm{kg}$. It is concluded that fatty foods such as döner should not be overcooked and contacted with flame directly.

\section{P42. Effects of short sleep duration on dietary intake and} obesity

\section{Lee $S^{\star}$, Doo M, Shin Y and Kim Y}

Department of Nutritional Science and Food Management, Ewha Womans University, Seoul, Republic of Korea, E-mail: hayeeun@empas.com
An association between short sleep duration and increased risk of obesity has been reported in recent studies. Short sleep duration may affect body mass index, appetite regulation, and a change in the composition of dietary macronutrients. The aim of this study was to examine the relationship between sleep duration and obesity-related variables or dietary macronutrients consumption as a novel risk factor for obesity in Korean. Study participants were 14,111 subjects aged 20-79 from the Korean National Health and Nutrition Examination Survey for 2010-2012. Relationship between sleep duration and obesity-related variables or dietary macronutrients intake was examined using multiple variable logistic regression models and general linear models after adjustment for age. Short sleep duration of less than 7 hours per day showed the results of increased body mass index, elevated plasma triglyceride level, and the prevalence of obesity for women. However, there was no significant correlation between sleep duration and body mass index, plasma triglyceride level, and obesity prevalence in men. Protein intake was significantly decreased in subjects with short sleep duration for both men and women. In contrast, carbohydrate consumption was significantly increased in women with short sleep duration, but not for men. Among women subjects whose carbohydrate consumption was above the median, subjects with short sleep duration showed their elevated odds of being obese (OR=1.25, 95\% CI: 1.07-1.46, $\mathrm{P}=0.028)$ compared with subjects with sleep duration more than 7 hours per day. Our findings support a significant association between sleep duration and obesity-related variables in Korean women. Especially, short sleep duration affected the increased dietary carbohydrate consumption, its influence lead to increased risk of obesity. Therefore, we cautiously suggest a potential that different sleep duration may be a cause of the differences between normal weight and overweight for Korean women.

\section{P43. The total phenols, lycopene contents and antioxidant} activities of some fruits and vegetables

\section{Zaki $\mathbf{S}^{1^{*}}$, Ezz Al-Arab ${ }^{2}$, Amin $\mathrm{W}^{1}$ and Nagi $\mathrm{H}^{1}$}

${ }^{1}$ Food Technology Department, Faculty of Agriculture, Cairo University, Egypt

${ }^{2}$ National Research Center, Dokki, Gizam Egypt, E-mail: drshafikazaki@gmail.com

A number of antioxidant nutrients and photochemical has been exhibited antioxidant properties when administered. Many natural products from vegetables, fruits, plant extracts, and herbs have been implicated in cancer prevention and that promote human health without side effects. The present investigation aimed to evaluate total phenols, lycopene contents and antioxidant properties of some dried fruits and vegetables (dried figs, apricots and raisins, carrots and tomatoes). These materials were chemically analyzed. The antioxidants activity, phenols and lycopene of the used materials were determined. The antioxidant activities of the vegetables and fruits assessed by the three different methods: 1 . a $\beta$-carotene/linoleic acid system 2. DPPH free radical scavenging assay 3 . Reducing power method. The highest values of antioxidant activity assessed by the three used methods were noticed for tomatoes, followed by those for dried figs and dried carrots, then raisins. In contrast, the least activities were found for the dried apricots. Total phenolic content of dried tomato $(162.9 \mathrm{mg} / 100 \mathrm{~g})$ was the highest and that of raisins $(45.6 \mathrm{mg} / 100 \mathrm{~g})$ was the lowest, but dried figs, carrots and apricots contained 148.6, 136.8 and $59.4 \mathrm{mg} / 100 \mathrm{~g}$, respectively. The data showed that dried tomato $(162.9 \mathrm{mg} / 100 \mathrm{~g})$ was the highest and raisins $(45.6 \mathrm{mg} / 100 \mathrm{~g})$ were the lowest, but dried figs, carrots and apricots were 148.6, 136.8 and $59.4 \mathrm{mg} / 100 \mathrm{~g}$, respectively. 
The highest content of lycopene was in dried tomatoes $(41.34 \mathrm{mg} / 100 \mathrm{~g})$, carrots had the lowest content $(1.32 \mathrm{mg} / 100 \mathrm{~g})$, while dried fruits did not contain lycopene.

P44. Vitamin-d3 upregulated protein-1(vdup1) is an antioxidant protein in immune cells under oxidative stress

Jung $\mathrm{H}^{1,2^{*}}$ and Choi $\mathrm{I}^{1,2}$

${ }^{1}$ Immunotherapy Research Center, Korea Research Institute of Bioscience and Biotechnology, Yuseong-gu, Daejeon 305-806, Republic of Korea

${ }^{2}$ Department of Functional Genomics, University of Science and Technology, Yuseong-gu, Daejeon 305-333, Republic of Korea, E-mail: haiyoung@kribb.re.kr

The regulation of ROS is an important process in determining cellular fate decisions under oxidative stress conditions (e.g., high glucose, infection or drugs). Vitamin-D3 upregulated protein1 (VDUP1) is known as a ROS regulator via direct interaction with TRX and a glucose uptake suppressor via internalization of glucose transporter 1(GLUT1) by direct interaction and reduction of GLUT1 expression. In this study, we issue that VDUP1 has an antioxidant function under oxidative stress. VDUP1 knockout mice exhibited elevated reactive oxygen species (ROS) and nitric oxide (NO) production in immune cells and were more susceptible to oxidant and endotoxin. These data indicate that VDUP1 plays a pivotal role in the protection of the immune cells as an antioxidant protein under oxidative stress and suggest that VDUP1 inducer, e.g., Vitamin D3, may regulate intracellular ROS levels by regulating the expression of VDUP1 in the immune cells during oxidative stress.

\section{P45. Monitoring of microorganisms in raw oysters of Korean} market

\section{Lee MJ`, Kim HJ, Kim JY, Kim JH, Lee HY, Shin YM and Yoon HS}

Ministry of Food and Drug Safety, Korea, E-mail: ymin0110@korea.kr

Raw oysters were analyzed for their microbial contamination (total aerobic bacteria, coliform, E. coli, S. aureus, V. parahaemolyticus, Salmonella spp.and L. monocytogenes). For this study, 150 samples were collected from Busan, Ulsan and Gyeongsangnam-do, South Korea. Experimental plans for microbiological test were in accordance with the International Commission on Microbiological Specifications for Food (ICMSF), excepted E. coli (MPN method, 30 samples). The contamination levels of total aerobic bacteria and coliform in sample were in range of $1.93 \sim 4.75$ and $0 \sim 2.72 \log$ CFU/g. E. coli was detected from 9 samples less than $230 \mathrm{MPN} / 100 \mathrm{~g}$. There were no detection of S. aureus, V. parahaemolyticus, Salmonella spp.and L. monocytogenes. The results of this study can be utilized as basic data for improving standards and specifications of Korea Food Code.

P46. Analysis of contribution rate of each Korean food group to calcium and phosphorus intake and calcium/phosphorus ratio

\section{Kim NY ${ }^{1}$, Shin $\mathrm{DM}^{1}$, Choi $\mathrm{MK}^{2}$, Park $\mathrm{HR}^{3}$, Heo $\mathrm{YR}^{4}$ and Lee $\mathrm{YK}^{1}$}

${ }^{1}$ Department of Food Science and Nutrition, Kyungpook National University, Daegu, Korea
${ }^{2}$ Department of Food and Nutrition, Kongju National University, Yesan, Korea

${ }^{3}$ Department of Food and Nutrition, Myongji University, Yongin, Korea

${ }^{4}$ Department of Food and Nutrition, Chonnam National University, Gwangju,Korea, E-mail:nd4222@naver.com

The daily calcium intake of Koreans in 2013 is $506.8 \mathrm{mg}$ (72\% of Recommended Nutrient Intake) and phosphorus intake is $1,114.2$ mg (163\% of Recommended Nutrient Intake). As high intake of phosphorus may hinder calcium absorption and cause osteoporosis, adequate phosphorus intake is needed. Therefore, to provide basic data required for nutrition education that increases calcium intake and reduces relatively high phosphorus intake, this study analyzed the contribution rate of each food group to calcium and phosphorus intake and calcium/phosphorus ratio. This study conducted two 24hour diet recall interviews with 640 adults ( 320 males and 320 females) from age 19 to 69 in each region (capital region, Chungcheong region, Gyeongsang region, Jeolla region), classified total 1,796 types of ingested foods into 31 groups, and analyzed the results using the nutritional evaluation program CAN-Pro 4.0. The contribution rate to calcium was highest in dairy products and frozen desserts at $12.60 \%(69.16 \pm 109.24 \mathrm{mg} / \mathrm{d})$, followed by stew and soup at $10.13 \%$ $(55.62 \pm 69.55 \mathrm{mg} / \mathrm{d})$. The contribution rate to phosphorus was highest in rice at $14.92 \%(60.69 \pm 107.09 \mathrm{mg} / \mathrm{d})$, followed by stir-fried foods at $8.21 \%(88.48 \pm 89.39 \mathrm{mg} / \mathrm{d})$. The groups with $\mathrm{Ca} / \mathrm{P}$ of 1 or above were pickled vegetables and salted dishes (1.27), kimchi (1.25), dairy products and frozen desserts (1.24), and vegetables and sea algae (1.05). Therefore, to increase calcium intake and reduce phosphorus intake of Koreans, it is necessary to reduce intake of food groups with high contribution rate to phosphorus. Considering that the recommended calcium/phosphorus intake ratio is $1: 1$, and the calcium/phosphorus ratio in foods with best calcium absorption is $1 \sim 2: 1$, it is necessary to provide education and publicity for people to increase intake of food groups with the $\mathrm{Ca} / \mathrm{P}$ ratio of $1 \sim 2$.

P47. Analysis of contribution rate of each Korean food group to sodium and potassium intake and ratio of sodium/ potassium

Kim NY ${ }^{1^{*}}$, Shin DM' ${ }^{1}$, Park HR ${ }^{2}$, Hyun $\mathrm{TS}^{3}$, Huh $\mathrm{YR}^{4}$, Ro $\mathrm{H} \mathrm{K}^{5}$ and Lee $\mathrm{YK}^{1}$

${ }^{1}$ Department of Food Science and Nutrition, Kyungpook National University, Daegu, Korea Korea

${ }^{2}$ Department of Food and Nutrition, Myongji University Yongin,

${ }^{3}$ Department of Food and Nutrition, Chungbuk National University, Cheongju, Korea

${ }^{4}$ Department of Food and Nutrition, Chonnam National University, Kwangju, Korea

${ }^{5}$ Department of Food Science and Nutrition, Dongshin University, Naju, Korea, E-mail: nd4222@naver.com

The purpose of this study was to analyze the contribution rate of each food group to sodium and potassium intake as well as sodium/ potassium ratio. This study conducted two 24-hour diet recall interviews with 640 adults ( 320 males and 320 females) from age 19 to 69 in 8 regions in Korea, classified total 1,796 types of ingested foods 
into 31 food groups, and analyzed the results using the nutritional evaluation program CAN-Pro 4.0. The contribution rate to sodium was highest at $11.25 \%$ by ingesting $(831.7 \pm 741.2 \mathrm{mg} / \mathrm{d})$ from noodles and dumplings, followed by $8.76 \%$ by ingesting $(647.4 \pm 477.2 \mathrm{mg} / \mathrm{d})$ from stew and soup. The contribution rate to potassium was highest at $7.98 \%$ by ingesting $(439.0 \pm 475.3 \mathrm{mg} / \mathrm{d})$ from cereals and roots/tuber crops, followed by $5.86 \%$ by ingesting $(322.4 \pm 249.7 \mathrm{mg} / \mathrm{d})$ from stew and soup. The $\mathrm{Na} / \mathrm{K}$ ratio was highest in salted seafood with $14.58 \pm 13.14$, and the food groups with the $\mathrm{Na} / \mathrm{K}$ ratio of 1 or below were fish meat (1.02), fat and oils (1.01), dairy products and frozen desserts (0.96), beverages and teas $(0.50)$, vegetables and sea algae $(0.40)$, beans.nuts.seeds $(0.13)$, rice (0.06), and fruits (0.06). The findings of this research also showed that the contribution rate to sodium intake was high in some of the food groups which also had high contribution rate to potassium intake, which indicates that it is suitable to use the sodium/potassium ratio along with the individual values of sodium and potassium in order to reduce sodium and increase potassium intake. In particular, it is necessary to provide education and publicity focusing on people in their 20 s and 30 s with high $\mathrm{Na} / \mathrm{K}$ intake ratio, so that they can ingest food groups with low $\mathrm{Na} / \mathrm{K}$ ratio.

P48. How much influence 'school lunch news' has been giving to the dietary behavior of elementary school pupils both in Japan and the United States

\section{Hasegawa $M^{\star}$, Nakagawa $Y$ and Karashima J}

Jissen Women's University, Japan, E-mail: hasegawa-megumi@ jissen.ac.jp

After world war 2nd, school lunch started with U.S. help in 1945 for the purpose of improving the nutrition situation on of Japanese pupils. Sixty five years after have passed since then, school lunch is now regarded as very important pillar for nutrition policies. The purpose of this study is put on investigating how much influence the 'School Lunch News' have been giving to the dietary behavior of pupils in both countries. The research was conducted both in Hachioji on the out skirt of Tokyo, Japan and Cincinnati, Ohio, U.S.A. The questionnaires were conducted for 64 pupils, grade 6 of H-elementary school in Hachioji and 68 pupils of S-elementary school in Cincinnati. Also another interview was conducted upon dietitian officials both in Japan and U.S.A. As the result of the questionnaires it was found that pupils were biplaized into 2 groups. One is 'the School Lunch News reading group' and the other is 'non-reading group'. This results shows that there is no definite evidence between 'school lunch behavior' an 'School Lunch News' but there was a sort of reciprocal relationship between 'checking the school lunch menu' and 'School Lunch News' in Japan. In the United States, there was considerable relationship between 'checking the school lunch menu' and 'School Lunch News'. Judging from what I have investigated, 'School Lunch News' is seems to be something effective for the level up of 'Nutrition Education' among pupils in both countries.

P49. Black rice (oryza sativa, heukmi) extracts stimulate osteogenesis but inhibit adipogenesis in mesenchymal c3h10t1/2 cells

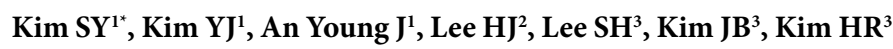
and Lee $\mathrm{SJ}^{1}$

${ }^{1}$ Department of Biotechnology, Graduate School of Life Sciences and
Biotechnology, Korea University, Seoul 136-713, Korea

${ }^{2}$ Department of Food and Nutrition, Eulji University. Seongnam-si, Gyeonggi-do, 461-713, Korea

${ }^{3}$ National Academy of Agricultural Science, Rural Development Administration, 166, Nongsaengmyeong-ro, Iseo-myeon, Wanju Gun, Jeollabuk-do, 565-851, Korea, E-mail: sungjoon.lee@gmail.com

Heukmi (Oryza sativa, Heukmi) is black-colored rice containing various bioactive compounds. We investigated the effects of black rice extracts (BREs) on osteogenic and adipogenic differentiation in C3H10T1/2 mesenchymal stem cells. BREs stimulate osteogenesis, enhancing the mRNA expression of runt-related transcription factor 2 and alkaline phosphatase (ALP), increasing ALP protein expression and cellular enzyme activity, thus elevating intracellular calcium deposition in C3H10T1/2 cells. BREs also suppressed adipogenesis in C3H10T1/2 cells, by downregulating peroxisome proliferator-activated receptor- $\gamma$, CCAAT-enhancer-binding protein $(\mathrm{C} / \mathrm{EBP}) \delta$, and $\mathrm{C} / \mathrm{EBP} \alpha$, major regulators in adipogenesis. In cells stimulated with BREs, the rate of fatty acid synthesis was significantly decreased while there was no effect on the rate of fatty acid oxidation, resulting in reduced of intracellular lipid accumulation. This reciprocal regulation of osteogenesis and adipogenesis by BREs was achieved via regulation of the Wnt signaling pathway. BREs enhanced the expression of genes in Wnt signaling pathway, including Wnt3a and $\beta$-catenin. Together, these results suggest that BREs significantly enhance osteogenic differentiation and suppress adipogenic differentiation. Induction of Wnt3a and $\beta$-catenin genes may be responsible for these effects. Thus, intake of BREs may improve bone health and attenuate bone lipid accumulation.

P50. Effects of subchronic administration of marine polyphenol phlorotannins on sleep profiles in mice

\section{Yang $\mathrm{HJ}^{\star}$, Yoon MS, Kim JY and Cho SM}

Korea Food Research Institute, E-mail: Yang.Hye-Jin@kfri.re.kr, eehdvkfl31@gmail.com

In neuropharmacology, polyphenols of herbal medicines have been considered as an important sedative-hypnotic compound. In particular, studies on the sedative-hypnotic effects of polyphenols have been limited to terrestrial plants. In our previous studies, it was demonstrated that marine polyphenol phlorotannins have sleeppromoting effects via positive allosteric modulation of the GABA type A-benzodiazepine receptors. Phlorotannins are oligomers and polymers of the monomeric unit phloroglucinol (1,3,5-tri-hydroxybenzene) and structurally different from the polyphenols of terrestrial plants, which typically contain gallic acids or flavones. In order to evaluate side effects, tolerance, and dependence of the hypnotic phlorotannins, we investigated effects of subchronic administration (3 weeks) of marine polyphenol phlorotannins on sleep profiles in C57BL/6N mice using analysis of electroencephalograms (EEG) and electromyograms (EMG). During 3 weeks, administration of PRT $(500 \mathrm{mg} / \mathrm{kg})$ significantly decreased the sleep latency and increased the total amount of non-rapid eye movement sleep (NREMS) duration compared to the vehicle. These effects were observed on the first and persisted until the last day during 21 days. However, the reduction effect of sleep latency and NREMS duration suppressed during administration of DZP (2 $\mathrm{mg} / \mathrm{kg}$ ). The DZP showed that delta activity decreased on the first day, but similar to vehicle on the last day and second day of withdrawal period. On the other hand, the EEG delta activity between the PRT treatment and the vehicle was no significant difference on the first day, and that was persisted throughout the whole treatment period. 
In conclusion, we demonstrated that PRT did not induce tolerance phenomena during subchronic administration for 3 week nor adverse effects at withdrawal. These results suggest that PRT can be potentially used as natural sleep aids without side effects for a long time.

\section{P51. Oxidation of flax seeds powder during storage time}

\section{Saphier $\mathrm{O}^{1^{*}}$, Silberstein $\mathrm{T}^{2}$, Tavor $\mathrm{D}^{1}$ and Yoshvayov $\mathbf{R}^{1}$}

${ }^{1}$ Department of Chemical Engineering, Sami Shamoon College of Engineering, Beer-Sheva, Israel

${ }^{2}$ Department of Gynecology and Obstetrics, Soroka University Hospital, Ben-Gurion University of the Negev, Beer-Sheva, Israel, E-mail: oshras@sce.ac.il

Introduction: As part of maintaining a healthy lifestyle and due to a growing awareness of the relationship between modern diet and appearances of various diseases, there has been interest in the nutritional values of flax seed. Flax seeds contain fiber, protein and minerals in high amounts that can assist and to contribute to human diet. Moreover, flax seeds are rich in antioxidants and polyphenols from the oil-rich fatty acids, containing alpha linolenic acid, ALA. Combination of omega-3 fatty acids with polyphenols has high positively benefits affecting various diseases, ranging from heart and blood vessels to cancer. However, a high content of fatty acid, flax seed makes food more sensitive to environmental conditions, which tends to oxidize fatty acid and be affected thereby impair the nutritional values of flax seed, and their impact on human nutrition. Aim of the study: To examine the aging process of flax seed powder, in order recommend storage conditions and maintenance of seed powder. Experimental: During research, we followed the change of polyphenols levels, and lipid peroxidation during long time of storage of seeds powder at different conditions. Determination of total phenols: The total phenolic contents in the extracts were determined using the Folin-Ciocalteu method. Fatty acid oxidation products were measured by analyzing the product Malondialdehyde, MDA in flax, using TRABS, Thiobarbituric acid reactive-substances method. The process of aging takes about 100 days, as during this time period, flax seed powder were exposed to different environmental conditions; in a closed container, at room temperature or refrigerator $4^{\circ} \mathrm{C}$, light or dark conditions. We also examined the influence of extreme storage conditions; Open container with light and ambient temperature. Results and conclusions: It was found that storage of flax seed powder in a closed container is possible at least for a period of 50 days, without incurring oxidation of fatty acids. In $4 \mathrm{oC}$, and dark, it is possible to extend the storage period for 65 days. About polyphenols level during storage, the main conclusion is that they have important role in the initial defense against oxidation of fatty acids. As time passes, their efficiency decreases and at the same time there may be an increase in their level. Paradoxically, after decreasing levels of polyphenols, for 20 days, there is an elevation in polyphenols level. We suggest that they may synthesize de-novo by the seeds powder in response to oxidative stress. This work has contributed greatly to the study of aging and shelf life of edible flax seed powder.

P52. The evidence for cardiovascular benefits of omega-3 fatty acids is insufficient to justify indiscriminate supplementation

\section{Pinchuk $I^{\star}$, Leshno M, Goldbourt U and Lichtenberg D}

Tel Aviv University, Sackler School of Medicine, Tel Aviv, Israel, E-mail: ilyap@post.tau.ac.il

Omega-3 is one of the most-studied food supplements. For many years, it has been regarded a scientifically proven supplement.
Recent results raised doubts whether the health benefits of omega-3 justify its indiscriminate use and in spite of the extensive research, supporters and opponents of omega 3 supplements keep arguing, on the basis of both new data and new analyses of old data, whether the available evidence justifies indiscriminate supplementation. Unlike previous meta-analysis, we considered only randomized, double blind, placebo-controlled clinical trials for the cardiovascular outcome of omega 3 capsules and analyzed the dependence of the results of these trials on the fraction of statin-treated participants. This dependence yielded estimates of the relative risk of cardiac events in statin-treated participants as well as in participants that did not take statins. The major conclusion of this analysis is that statin-treated people are hardly affected by omega 3 supplementation, whereas the risk of cardiac death in people receiving no statins is about $10 \%$ lower in omega 3 -supplemented people than in participants of a control group that do not get omega 3. Analyses of the results in terms of a Markov model indicates that omega 3 supplementation prolongs the quality-adjusted life years (QALY) by about a month, depending on age and health state. Given the pronounced benefit of hyper-triglyceridemic people, normolipidemic people that are not treated with statins may gain from omega 3 supplementation up to one month. Given the strong evidence for the benefit of statins and the results of our analysis, we do not recommend indiscriminate supplementation of omega 3.

\section{P53. Genomic characterization of foodborne pathogens}

\section{Amoako $K^{\star}$, Janzen T, Goji N and Thomas M}

Canadian Food Inspection Agency, E-mail: kingsley.amoako@ inspection.gc.ca

Microbial typing provides important information, which when combined with epidemiological evidence could determine the source of a foodborne human pathogen and its tracking to a contaminated food source. Current genotyping methods (e.g. Pulse Field Gel Electrophoresis (PFGE)) do not have the resolution needed to discriminate between closely related strains of a number of pathogens nor the ability of analysing genomic diversity. This necessitates the need for development of novel technology platforms with higher resolution for foodborne pathogen detection and characterization. Argus Whole Genome Optical Mapping (WGOM) is a rapid de novo process that generates whole genome, ordered restriction maps with no reliance on sequence information. These optical maps can subsequently be used for source tracking, gene variant detection, and assisting in next generation sequencing data assembly. In this study we applied the use of this technology for high resolution genotyping of foodborne pathogens including Listeria monocytogenes, Salmonella enterica serovars, and Escherichia coli. The Whole Genome Optical Maps provide high resolving power and detail genomic differences between the Listeria, Salmonella, and E. coli isolates. The information generated demonstrates the potential application for tracking foodborne disease outbreaks and identifying the source of foodborne pathogens. Our results suggest that differences determined by PFGE may not always completely reflect the genetic rearrangements. This technology platform generates whole genome optical maps within 24 hours, presenting an alternative to other existing typing methods and can also aid in next generation sequence assembly.

P54. Schizandra chinensis improved osteoporosis by activating estrogen receptor- $\alpha$ and $-\beta$

Yang $\mathrm{WM}^{*}$, Kim MH and Lee HS

College of Korean Medicine and Institute of Korean Medicine, 
Kyung Hee University, Seoul 130-701, Korea,E-mail:wmyang@ khu.ac.kr

Osteoporosis is a critical bone metabolic disease in estrogen depleted women after menopause. The fruit of Schizandra chinensis (SC) contains several lignans, which may be phytoestrogen. The purpose of this study was to investigate anti-osteoporotic effects of SC in vivo and in vitro. Seven weeks ICR female mice were ovariectomized and recovered to induce osteoporosis for 7 weeks. The $70 \%$ ethanol extract of SC was administrated orally for 6 weeks after the recovery period from osteoporosis induction. Bone mineral density was significantly increased following increased serum osteocalcin levels by SC treatment. SC also reduced growth plate hypertrophy and increased interstitial cells in the spongiosa area of metaphysis of the femoral head. In addition, there was a decrease in the number of pores within bone marrow cells. The serum estradiol concentration was significantly increased in the SC group. The expression of estrogen receptor- $\alpha$ and $-\beta$ was increased in the uterus and MCF-7 breast cancer cell, and transcription of two proto-oncogenes, c-fos and c-Jun, was not detected after treatment of SC. From these data, we suggest that SC might improve osteoporosis due to its phytoestrogenic effects, demonstrating the potential effects of SC on alternative medicine for treating osteoporosis.

P55. Ascorbic acid content and nitrate of the aerial part of sweet basil (ocimum basilicum 1.) Grown in pots

Muráriková $\mathrm{A}^{\star}$ and Neugebauerová J

Mendel University in Brno, Faculty of Horticulture, Department of Vegetable Growing and Floriculture, Czech Republic, E-mail:xmurarik@ node.mendelu.cz

This study was an investigation to compare the content of ascorbic acid (AA) and nitrate in 7 sweet basil (Ocimum basilicum L.) varieties grown in pots. Experiment was conducted from 23rd April to 23rd June in the greenhouse of Faculty of Horticulture, Mendel University in Brno. All the plants were harvested 8 weeks after sowing. Aerial parts were evaluated immediately after harvest. The AA content was determined by High Performance Liquid Chromatography (HPLC). The highest average AA content was confirmed in variety 〈Ohře〉 (181.9 mg.kg-1 f. w.) and lowest in variety 〈Mánes〉 (87.0 mg.kg-1 f. w.). Our results prompt the conclusion that fresh aerial parts of basil are a good source of vitamin $\mathrm{C}$ and are suitable for diet. The nitrate content was determined by Ion Selective Electrode (ISE). The content of nitrate ranged from 1230.1 to $178.7 \mathrm{mg} . \mathrm{kg}-1 \mathrm{f}$. w. with the highest content in variety «Red Rubin` and lowest content in variety «Mammolo Genovese>. Maximum acceptable content of nitrate $(2500 \mathrm{mg}$ NO3. kg-1) for summer crops (European Commission, 1997) was reached in any examination of basil varieties. This means that maximum concentration of nitrate found in this study (1230.1 mg.kg- $1 \mathrm{f}$. m.) does not represent risk for human consumption.

P56. Human placenta induced hair regrowth in chemotherapyinduced alopecia

\section{$\operatorname{Kim} \mathrm{K}^{1^{*}}$, Kim $\mathrm{MH}^{2}$ and Yang $\mathrm{WM}^{2}$}

${ }^{1}$ Department of Ophthalmology, Otorhinolaryngology and Dermatology of Korean Medicine, College of Korean Medicine, Kyung Hee University, Seoul, Republic of Korea

${ }^{2}$ College of Korean Medicine and Institute of Korean Medicine,
Kyung Hee University, Seoul 130-701, Korea, E-mail: kmdkskim@khu. $a c . k r$

Human placenta extract (HPE) has been used for tiredness and anti-aging in Korean medicine. However, its mechanism is not fully understood yet despite several studies on the hair growth of HPE. This study aimed to understand the molecular mechanisms of HPE on hair growth-promoting activity in chemotherapy-induced alopecia. To induced dystrophic catagen development accompanied with apoptosis, C57BL/6 mice in telogen were depilated to enter anagen. On 9 day after depilation, mice were injected $150 \mathrm{mg} / \mathrm{kg}$ cyclophosphamide. Dystrophic hair follicles by chemotherapy entered spontaneously into catagen on 17 days after depilation. Mice were topically treated with 1 and $100 \mathrm{mg} / \mathrm{mL}$ HPE to the dorsal skin on 9-17 days. Miniaturized hair follicles were recovered by treatment of HPE. Topical HPE treatment induced newly straight hair shafts emerged from hair follicles containing hair fibers. The expression of caspase- 3 was decreased by HPE treatment. In HPE-treated skin region, there was an increase in $\mathrm{Bcl}-2 / \mathrm{Bax}$ ratio by down-regulating the expression of pro-apoptotic Bax rather than anti-apoptotic Bcl-2 expression. These results lead to a decrease on the release of cytochrome c into cytoplasm. In summary, HPE restored apoptosis-regression dystrophic catagen in hair follicles, resulting in hair regrowth. HPE may be used for alternative treatment of chemotherapy-induced alopecia.

P57. Topical treatment of angelica sinensis ameliorated ligature-induced periodontitis via an inhibition of collagenase mmp-9

\section{Choi $\mathrm{YY}^{\star}$, Lee HS and Yang WM}

College of Korean Medicine and Institute of Korean Medicine, Kyung Hee University, Seoul 130-701, Korea, E-mail: c9640@khu.ac.kr

Periodontitis is a chronic disease associated with excessive tissue destruction including tooth-supporting structures and gingival tissues. Angelica sinensis has been reported to have several biological effects. However, there are no studies regarding on inhibitory effects of A. sinensis on periodontitis. In this study, ameliorative effects of $50 \%$ ethanol extract of A. sinensis (AS) against ligature-induced periodontitis were investigated. Sprague-Dawley (SD) rats were divided four groups; nonligated (normal), ligated and treated vehicle (control), ligated and treated $1 \mathrm{mg} / \mathrm{mL}$ AS (AS1), ligated and treated $100 \mathrm{mg} / \mathrm{mL}$ AS (AS100). 1 and $100 \mathrm{mg} / \mathrm{mL}$ AS were topically treated onto both side of first molar daily. After 2 weeks, mandibles and gingival tissues were collected.

Topical AS treatment inhibited the alveolar bone loss as shown in methylene blue staining. AS-treated group restored the infiltrate gingival tissues and cementum, indicating that AS recovered the structures of the molar. Decreases in osteoclast cells stained TRAP positive were observed by treatment of AS. To confirm the mechanism of protective effects of AS on periodontitis, the expression of collagenase in gingival tissues was investigated. The expression of MMP-9 mRNA was decreased in AS-treated gingival tissues. These results revealed that AS exhibited its therapeutic effects on periodontitis via an inhibition of collagenase MMP-9 expression. AS may be an alternative remedy for treating periodontitis.

P58. Lycium chinense inhibited oxidative stress in hydrogen peroxide-induced tm 3 leydig cells

\section{Kim MH*, Choi YY and Yang WM}

College of Korean Medicine and Institute of Korean Medicine, 
Kyung Hee University, Seoul 130-701, Korea, E-mail: kimmihye526@ gmail.com

Lycium chinense (LC) is used as functional food as well as medicinal herb. However, its antioxidant activity on testicular Leydig cells has not been investigated yet. In the present study, we assessed the cytoprotective effects of LC against hydrogen peroxide ( $\mathrm{H} 2 \mathrm{O} 2)$ induced oxidative stress in TM3 Leydig cells.

To induce the oxidative stress, TM3 Leydig cells were stimulated with $\mathrm{H}_{2} \mathrm{O}_{2}$ for $24 \mathrm{~h} .1,10$ and $100 \mu \mathrm{g} / \mathrm{mL}$ LC were pre-treated for $18 \mathrm{~h}$ and co-treated with $\mathrm{H} 2 \mathrm{O} 2$ for $24 \mathrm{~h}$. The survival rate of TM3 Leydig cells was measured using 3-(4,5-dimethylthiazol-2-yl)-2,5-diphenyltetrazolium bromide, a yellow tetrazole. Testosterone enzyme immunoassay was carried out. In addition, phosphorylation of cell proliferation-related markers as well as oxidant injury markers was shown by western blot analysis.

Decreased cell viabilities induced by $\mathrm{H}_{2} \mathrm{O}_{2}$ were significantly increased following no significant cytotoxicity by LC treatment. Marked reduction of testosterone was induced in TM3 cells with $\mathrm{H}_{2} \mathrm{O}_{2}$. Treatment of various concentrations of LC restored the testosterone production in dose-dependent manner. In addition, the expressions of inducible nitric oxide synthase and cyclooxygenase-2 were suppressed with treatment of LC. Furthermore, LC treatment leads to cell proliferation against oxidative stress by up-regulating the phosphorylation of extracellular signal-regulated kinase $1 / 2$ and protein kinase B.

Taken together, these findings indicated that LC exerted the protective effects on functions of TM3 Leydig cells from $\mathrm{H} 2 \mathrm{O} 2$-induced oxidative stress.

\section{P59. The content of vitamin $\mathrm{E}$ in different types of nuts}

\section{Táborský J*, Hejtmánková A, Kudelová V and Popov M}

Department of Chemistry, Faculty of Agrobiology, Food and Natural Resources, Czech University of Life Sciences Prague, E-mail: taborsky@ af.czu.cz

During the whole history of mankind, nuts have been very important part of food thanks to their good nutritional composition1. A high content of fat in the nuts (46-76\%) is associated with a significant content of vitamin $\mathrm{E}$ belonging to the major antioxidant substances. Vitamin $\mathrm{E}$ includes eight species of tocols, $\alpha, \beta, \gamma, \delta$-tocopherols and tocotrienols. The highest biological activity is attributable to $a$-tocoferoll. At present, however, the great attention is paid to the other tocols mainly to their hypocholesterolemic, neuroprotective and antitumor impact2,3. According to some studies, $\gamma$-tocoferol4 and tocotrienols5 may exhibit much higher biological activity than a-tocopherol, which is often a single ingredient of vitamin E1. The aim of this study was to identify all forms of vitamin $\mathrm{E}$ in different kinds of nuts obtained from different sources and their mutual comparison. All tocols were determined by reversed phase HPLC using isocratic elution with fluorescence detection. As the most important source of a-tocopherol were evaluated pistachios (average value $1132 \mu \mathrm{g} . \mathrm{g}-1$ ), $\beta$-tocopherol was present in all samples as a minor component, its maximum content was found in hazelnuts $(9.6 \mu \mathrm{g} . \mathrm{g}-1) . \gamma$-tocopherol was found in all kinds of nuts and the highest content was found again in pistachios $(584 \mu \mathrm{g} . \mathrm{g}-1)$, very high amounts of $\delta$-tocopherol were contained in Brazil nuts (2297 $\mu$ g.g-1). Tocotrienols were found in a smaller number of nut kinds than tocopherols. $\alpha$-tocotrienol was found only in three kinds of nuts - Brazil nuts, which contained the highest amount (399 $\mu \mathrm{g} . \mathrm{g}-1)$, pine nuts and hazelnuts grown in the Czech Republic, which have been analyzed soon after harvest. The content of $\gamma$-tocotrienol was also determined only in three kinds of nuts (pistachios, macadamias, and walnuts grown in the Czech Republic, which were analyzed soon after harvest), the most significant amount was found in pistachios $(34,8 \mu \mathrm{g} . \mathrm{g}-1) . \beta$ and $\gamma$-tocotrienols were below the detection limit. In the Czech Republic, walnuts and hazelnuts are the most widely cultivated and consumed nuts. While in walnuts the most important form is $\gamma$-tocopherol ( $459 \pm 40 \mu \mathrm{g} . \mathrm{g}$ - 1$)$, in hazelnuts it is $\alpha$-tocopherol $(876 \pm 151 \mu \mathrm{g} . \mathrm{g}-1)$.

\section{P60. Determination of fatty acid composition in nut oils}

\section{Táborský J*, Cejpková $\mathrm{M}$ and Hejtmánková $\mathrm{A}$}

Department of Chemistry, Faculty of Agrobiology, Food and Natural Resources, Czech University of Life Sciences Prague, E-mail: taborsky@ af.czu.cz

Nuts are valued for their sensory and nutritional attributes, especially for the high content of oil, rich in unsaturated fatty acids, which are important in human nutrition. The aim of this study was to determine and compare the fatty acids profiles in the oils of different nut types which are commonly available in the Czech Republic. Eight types of nuts were chosen for the purposes of this study (hazelnuts, cashews, macadamia nuts, almonds, Brazil nuts, pecan nuts, pine nuts and walnuts). These were analysed in three series, procured in three different sale locations. The oils were isolated by cold extraction with the use of hexane; fatty acids were trans-esterified to methyl esters, and these samples were analysed by gas chromatography with a flame ionization detector. Across all the different nut types, monounsaturated and polyunsaturated fatty acids were found to have the highest percentage in the extracted oils, ranging from $75 \%$ (Brazil nuts) to $90 \%$ (hazelnuts, almonds, walnuts). Of that percentage, oleic and linoleic acids were the highest contributors in most of the nut types. Oleic acid ranged from $17.6 \%$ (walnuts) to $80.9 \%$ (hazelnuts), with linoleic acid ranging from $1.8 \%$ (macadamia nuts) to $59 \%$ (walnuts). In macadamia nuts, palmitoleic acid percentage was found to be far higher than in any other nut type. Similarly, walnuts exhibited comparatively highest percentage (across nut types) of $\alpha$-linolenic acid (12.9\%). The highest percentage of saturated fatty acids was found in Brazil nuts (23.5\%), while the lowest was found in pine nuts $(6.8 \%)$. These results confirm the recommendation of nutritionists that nuts or nut oils are important sources of health beneficial unsaturated fatty acids (oleic, linoleic and in walnuts also $a$-linolenic acid), although amounts of individually acids significantly differ across nut types. Regarding also to their contents of phytosterols and antioxidants, some types of nuts can be used, when moderately included in a diet, to prevent risks of cardiovascular diseases.

\section{P61. Dietary polyamines and obesity: polyamine metabolic} enzymes involve in obesity

\section{Buyukuslu N*, Hizli H and Yoldas H}

Department of Nutrition and Dietetics,Faculty of Health Sciences,Istanbul Medipol University, Turkey, E-mail: nbuyukuslu@ medipol.edu.tr

The natural polyamines, putrescine, spermidine and spermine are distributed widely in all cells including adipocytes and involved in several physiological processes including gene expression and cell proliferation. The body pool of polyamines is maintained by endogenous biosynthesis, intestinal microorganisms, and the diet. 
Obesity is associated with the excess fat stored in adipocytes. Polyamine metabolism and its inhibition have been associated with increased adipose tissue and weight gain in human and animal models. Ornithine decarboxylase (ODC) and S-adenosylmethionine decarboxylase (AdoMetDC) are anabolic enzymes and spermidine/ spermine N1acetyltransferase (SSAT) and polyamine oxidase (PAO) are catabolic enzymes which regulate polyamine homoeostasis. Knockout SSAT decreased fatty acid catabolism, increased tissue adipose content, and increased weight gain indicating potential links between obesity and polyamine inhibition. Moreover, in Zucker rats, obesity was associated with the increased concentrations of endogenous spermine and spermidine. In mice on a high-fat diet, a deletion of SSAT exaggerated weight gain and the transgenic overexpression suppressed weight gain. The disruption of spermine synthase gene exhibited smaller size and lower weight than normal mice, whereas spermine synthase overexpressing mice were slightly larger than normal mice. It was suggested that not only spermine/spermidine ratios but also polyamine metabolic enzyme activities may contribute to adipogenesis. The regulatory enzyme AdoMetDC catalyzes the decarboxylation of S-adenosyl methionine (SAM) which is committed to polyamine bio-synthesis. Any disruption in decarboxylation of SAM may cause abnormal gene expression which is implicated in obesity. In addition, a study in urban Indian children showed the association of AdoMetDC variant with obesity. Human and animal studies have indicated that human milk has increased concentrations of polyamines in serum in obese children. It is obvious that there has been a correlation between fat metabolism and polyamine metabolism however further researches are needed to explore the influence of dietary polyamines on obesity.

P62. Comparative studies on biological activities of crude extracts in Korean colored rice

\section{Han $S^{\star}$, Park D, Seo W, Ra J, Song Y, Nam M and Kwon Y}

National Institute of Crop Science, RDA, South Korea, E-mail: han0si@korea.kr

Rice is consumed as a staple food by over one-half of the world's population and represents one of the most important food crops worldwide. Asian consumers require health rice cultivars and make purchase decisions with high criteria of healthy functions in rice foods. The growing interests in functional foods have intensified the research on the potential of rice as a source of bioactive micronutrients. To cope with these need, the four colored rice (Oryza sativaL.) harvested in Korea were used as research material throughout all the experiments including white (control), brown, red and black waxy giant embryo rice cultivar (BGE) as a functional rice. Bioactive constituents were determined and measured their contents from water, methanol and lipid extracts. Significant variation in functional substances (such as amino acid, poly phenol, flavonoid, fatty acid and $\gamma$-oryzanols) was observed in tested rice cultivars. The synthesis and accumulation of triglyceride (a risk factor of heart disease in blood stream) by fatty 3T3-L1 cells were suppressed $64.2 \%$ by the treatment of water extracts from BGE, which was 2 folds higher than that of methanol extracts. In addition, anti-inflammatory activity was observed from the test of mRNA expression with TNF- $\alpha$. IL-6, IL-1 $\beta$, and PGE2. Within the test, methanol extracts from black rice showed significant inhibition of expression of these cytokines compared to other cultivars. These findings indicate that black waxy giant embryo rice posses significant lowering effect of triglyceride and anti-inflammatory activities, simultaneously. Consumption of colored rice rich in beneficial bioactive compounds may be a useful dietary strategy for achieving optimal health.
P63. Methyl syringate as an appetite suppressant via the transient receptor potential channel ankryn 1 (trpa1)

\section{Kim MJ ${ }^{\star}$, Son HJ and Rhyu MR}

\section{Korea Food Research Institute, E-mail: mjkim14@kfri.re.kr}

Gastrointestinal (GI) tract regulates gastric motility and gastric emptying which is related to gastroparesis as well as appetite, food intake, and body weight. In addition, GI hormones including peptide YY (PYY) and glucagon-like peptide 1 (GLP-1) are important factors to control food intake and gastric emptying. The increment of plasma PYY (3-36) and GLP-1 enhance c-fos expression and delay gastric emptying. Somatosensory transient receptor potential channel ankryn 1 (TRPA1) is usually expressed in sensory neuron, but also in enteroendocrine cells in the gastrointestinal (GI) tract. Therefore, TRPA1 agonist may affect the functions of GI tract. In previous study, we have shown that methyl syringate, one of the pungent ingredients in Kalopanax pictus Nakai (K. pictus), acted as a TRPA1 agonist. In this study, we investigated the effects of methyl syringate on food intake, gut hormone levels, and gastric emptying in imprinting control region (ICR) mice. At $24 \mathrm{~h}$ after administration of methyl syringate $(10 \mathrm{mg} / \mathrm{kg})$, cumulative food intake and gastric emptying in ICR mice were significantly suppressed. Ruthenium red (RR, $0.33 \mathrm{mg} / \mathrm{kg}$ ), a general cation channel blocker, and HC-030031 (0.15 mg/kg), a selective TRPA1 antagonist effectively inhibited methyl syringate-induced suppression of cumulative food intake and gastric emptying. Furthermore methyl syringate (6.5 and $10 \mathrm{mg} / \mathrm{kg}$ ) effectively increased plasma PYY (3-36) level, but these phenomena were inhibited by RR $(0.33 \mathrm{mg} / \mathrm{kg})$ and HC-030031 $(0.15$ $\mathrm{mg} / \mathrm{kg}$ ). On the other hand, GLP-1 level was not changed by methyl syringate $(0.1-10 \mathrm{mg} / \mathrm{kg})$. These data suggest that methyl syringate, a TRPAl agonist can contribute to weight suppression.

\section{P64. Monitoring of microbiological contamination levels of ready-to-eat food products consumed in Korea}

\section{An ES*, Lee JK, Yoo MS, Park SJ, Ryu HY and Lim JH}

Hazardous Substances Analysis Division, Gwangju Regional Korea Food and Drug Administration, Gwangju, Korea, E-mail: jupiter12534@ gmail.com

The consumption of ready-to-eat food products has increased significantly in recent decades. All of these products in Korea Food Code were not applied to the regulation of the microbiological criteria using sampling plans recommended by The International Commission on Microbiological Specifications for Foods (ICMSF). Therefore we need to monitor the levels of microbial contamination in ready-to-eat food products by using scientifically proven techniques including statistical analysis. In this study, we investigated the microbial contamination (total aerobic counts, coliform group, Escherichia coli and food-borne pathogens) in ready-to-eat food products such as ready-to-eat foods, ready-to-cook foods, ready-to-eat fresh foods which were consumed in Korea. A total of 60 samples were collected according to the sampling plans recommended by ICMSF from a number of grocery stores and markets. All samples were analysed by using the method of Korea Food Code. The detection levels of the microbiological contamination in 20 ready-to-eat foods, 32 ready-to-cook foods and 8 ready-to-eat fresh foods ranged from Not Detected (ND) to $4.9 \mathrm{log} \mathrm{cfu} / \mathrm{g}$ for total aerobic bacteria, from ND to $1.5 \mathrm{log} \mathrm{cfu} / \mathrm{g}$ for coliforms, and from ND to 2.2 $\log \mathrm{cfu} / \mathrm{g}$ for B. cereus. But E. coli, S. aureus, C. perfringens and V. parahaemoliticus were not detected in all samples. These monitoring results will be used as fundamental data to reestablish microbiological 
criteria of Korea Food Code to harmonize international food standards.

P65. Protective effect of comp-angiopoietin-1 in inflammation-associated lymphangiogenesis in dextran sulfate sodium (dss)-induced colitis model

\section{Lee $\mathrm{AS}^{*}$}

Korea Food Research Institute, 516 Baekhyun-Dong, Bundang-Ku, Seongnam Gyeonggi 463-746, Republic of Korea, E-mail: aslee@kfri.re.kr

Ulcerative colitis is an inflammatory disease that is condition of the colon and small intestine. Animal models of colitis required administration of specific concentrations of colitis-inducing chemicals, such as dextran sulphate sodium (DSS). COMP-angiopoietin1(COMP-Ang1) was generated by replacing the $\mathrm{N}$-terminal portion of Ang1 with the short coiled-coil domain of cartilage oligomeric matrix protein (COMP), had a soluble, stable, and potent Ang1 variant. It is reported that blood serum angiopoietin-1 levels elevated in ulcerative colitis (UC) patients, as a progression factor in the development of inflammatory bowel disease (IBD). In this study, systemic delivery of COMP-Ang1 ameliorated body weight loss, DAI, shortening and histopathological score of the colon in a DSS-induced colitis model. Furthermore, COMP-Ang1 reduced the expression of pro-inflammatory cytokines and vascular endothelial growth factor (VEGF)-A, C and D, is correlated with inflammation-associated lymphangiogenesis. Treatment of COMP-Ang1 led to reduced infiltration of leukocytes to the inflamed colon. And we evaluated the expression of classically activated (M1) macrophages and alternatively activated (M2) macrophages in DSS-colitis model. Consequently, COMP-angiopoietin-1 has potential application in conditions marked by inflammation-associated lymphangiogenesis and ulcerative inflammation.

\section{P66. Flavonoids as prooxidants}

\section{Krych-Madej J* and Gebicka L}

Institute of Applied Radiation Chemistry, Faculty of Chemistry, Lodz University of Technology, Wroblewskiego 15, 93-590 Lodz, Poland, E-mail: jkrych@mitr.p.lodz.pl

Flavonoids are a group of plant polyphenols with different chemical structure and properties. Being widely distributed in fruits, vegetables, seeds, nuts, and beverages such as red wine and tea, they are an integral part of human diet. Flavonoids are known as efficient antioxidants. In vitro studies have shown that they efficiently scavenge free radicals and other oxidants, and are able to chelate metal ions. On the other hand, prooxidant activity of flavonoids has also been reported. This activity is mainly connected with the reactivity of reactive oxygen species formed during flavonoids oxidation, enzyme inhibition, and with the reactivity of oxidized flavonoids toward thiols and nucleic acids.

Recently we have shown that flavonoids inhibit catalase, one of the main enzymes of the antioxidant defense system of the cell, which decomposes hydrogen peroxide $(\mathrm{H} 2 \mathrm{O} 2)$ to water and molecular oxygen. The most potent catalase inhibitors among the tested flavonoids are myricetin, epicatechin gallate, and epigallocatechin gallate. There are even more efficient than well-known catalase inhibitor, azide. In the presence of flavonoids and under low fluxes of $\mathrm{H} 2 \mathrm{O} 2$ catalase is converted to its inactive form - Compound II. We have found that preincubation of catalase with some flavonoids before hypochlorous acid (HOCl) treatment significantly sensitizes this enzyme to the $\mathrm{HOCl}$ action. The consequences of catalase-flavonoid interactions will be presented.
P67. Comparison of functional, antioxidant and aceinhibitory activities of sardine (sardinella pilchardus) viscera protein hydrolysates using proteases from brewers? yeast surplus (saccharomyces pastorianus) and commercial proteases

\section{Vieira $\mathrm{EF}^{\star}$ and Ferreira I}

Department of Bromatology and Hydrology of Faculty of Pharmacy, University of Porto, Portugal, E-mail: elsavieiraf@gmail.com

In order to better utilize Sardinella pilchardus viscera, which is normally discarded as industrial waste in the process of fish manufacturing, hydrolysis of protein fraction was performed using different proteases: enzyme extract from spent brewer's yeast (Saccharomyces pastorianus); crude enzyme extract from sardine viscera; Alcalase ${ }^{\oplus}$; Neutrase $^{\oplus}$ and Flavourzyme ${ }^{\oplus}$. Treatments were performed independently at optimum temperature and $\mathrm{pH}$ conditions and at same E/S ratio (1:100) and time reaction (4 hours). Viscera protein hydrolysates were characterized with respect to protein recovery; hydrolysis degree; peptide molecular weight distribution (determined by size exclusion chromatography and SDS-PAGE analysis); antioxidant activities (DPPH radical-scavenging activity, ferric ion reducing antioxidant power and total phenolics); ACEInhibitory activity and functional properties (solubility, foaming, emulsifying ability). All sardine viscera hydrolysates showed different degrees of hydrolysis and different biological activities; the molecular weight distribution ranged between 20 and $<3 \mathrm{KDa}$. Flavourzyme ${ }^{\oplus}$ gave the highest yields of hydrolysis degree and FRAP antioxidant activity, $2.35 \mathrm{mM} \mathrm{TE} / \mathrm{mL}$ hydrolysate $(\mathrm{p}<0.05)$. Hydrolysates generated with crude enzyme extract from sardine viscera and with proteases from brewer's yeast extract displayed FRAP antioxidant activity of $0.76 \mathrm{mM}$ $\mathrm{TE} / \mathrm{mL}$ hydrolysate and $1.47 \mathrm{mM} \mathrm{TE} / \mathrm{mL}$ hydrolysate, respectively. The lowest ACE inhibitory activity was observed for sardine viscera hydrolysate produced by protease extract from brewing yeast surplus (1969.70 $\mu \mathrm{g}$ peptides $/ \mathrm{mL})$; Alcalase ${ }^{\oplus}$ and Neutrase $^{\oplus}$ treatments produced, respectively, sardine protein hydrolysates with ACE inhibitory activity of $119.27 \mu \mathrm{g}$ peptides/mL and $135.14 \mu \mathrm{g}$ peptides/ $\mathrm{mL}(\mathrm{p}<0.05)$.

These findings suggest that Sardinella pilchardus viscera byproduct is a good source of natural antioxidants and may be a beneficial ingredient to use in functional foods. Further work is therefore needed concerning sensory analysis of viscera hydrolysates.

P68. Physico-chemical characterization of antioxidative pecticpolysaccharide from mangosteen (garcinia mangostana) rind

\section{Tan SJ* and Gan CY}

Centre for Advanced Analytical Toxicology Services, Universiti Sains Malaysia,E-mail:szejack88@hotmail.com

Common non-starch polysaccharide materials were widely utilized in food and pharmaceutical industries. However, the attention has been shifted to utilizing agro-waste derived materials as alternative, versatile and biologically functional sources of polysaccharide. Pecticpolysaccharide extracted from Garcinia mangostana rind at different $\mathrm{pH}$ conditions $(\mathrm{MPpH} 2, \mathrm{MPpH} 3$ and $\mathrm{MPpH} 4)$ were characterized based on their physico-chemical properties in order to investigate the potential applications in the food industry. Results showed that extraction at $\mathrm{pH} 4$ gave the highest yield (21.1\%) and highest protein content $(2.9 \%)$ while extraction at $\mathrm{pH} 2$ gave highest purity $(76.8 \%)$ which fulfilled the Food Chemical Codex requirement. In terms of 
properties, it was found that this extract $(\mathrm{MPpH} 2)$ obtained a highest emulsification capacity $(81.3 \%)$ with the emulsion stability of $91.3 \%$. A thixotropic behaviour gel solution was produced using the same sample and $\mathrm{MPpH} 2$ gave the highest viscosity (viscosity decreased slightly from 1.784 to $1.322 \mathrm{~Pa} . \mathrm{S}$ ) compared to the extracts from $\mathrm{pH} 3$ and 4. High water- and oil-capacities ( 4.5 and $2.9 \mathrm{~g} / \mathrm{g}$ ) were also observed in the same sample. However, low solubility $(45.2 \%)$ was found in extract at $\mathrm{pH}$ 2. Functional group and degree of esterification (DE) studies showed that all three extracts were pectin-like polysaccharides. Apart from physico-chemical properties, all the extracts attributed to high antioxidative properties. MPpH2 consisted of $260.4 \mathrm{mg} \mathrm{GAE} / 100 \mathrm{~g}$ samples, whereas MPpH3 and MPpH4 consisted of 234.2 and 223.2 mg GAE/100 g of samples, respectively. High \%DPPHsc and FRAP values were observed in these samples as well. Having all the beneficial properties, pectic-polysaccharides from the Garcinia mangosteen rind has the potential to be imparted into the food system as functional polysaccharides.

P69. Cumin seed (cuminum cyminum), an alternative source of antioxidative and antidiabetic bioactive peptides

\section{Siow $\mathrm{HL}^{*}$ and Gan CY}

Centre for Advanced Analytical Toxicology Services, Universiti Sains Malaysia, 11800 USM, Penang, Malaysia,E-mail: candyshl89@gmail.com

Physiologically active peptides derived from food sources have gained mounting interest due to their therapeutic potential. Such peptides are inactive within the sequence, and however, they could trigger physiological action once released from their intact proteins. Bioactive peptides, which exhibited antioxidative and $\alpha$-amylase inhibition properties, were successfully extracted from cumin seeds using commercial enzyme. Based on a single factor experiment, hydrolysis parameters including incubation temperature, time and substrate-to-enzyme (S/E) ratio had a significant impact on the bioactivities of peptides. Incubation temperature of $50^{\circ} \mathrm{C}$, incubation time of $2 \mathrm{~h}$ and the use of S/E ratio of $20(\mathrm{w} / \mathrm{w})$ were demonstrated as the desired parameters in producing peptides with remarkable bioactivities in terms of DPPH scavenging abilities, ferric ion reducing power and a-amylase inhibitory activities. Sodium dodecyl sulfate-polyacrylamide gel electrophoresis (SDS-PAGE) profiles were performed to investigate the extent of protein hydrolysis under different conditions. Fractionation based on molecular mass was performed using ultrafiltration and fraction with $\mathrm{MW}<3 \mathrm{kDa}$, exerted the strongest radical scavenging activity (32.26\%DPPHsc/ $\mu \mathrm{g})$, reducing power $(11.56 \mathrm{mM} / \mu \mathrm{g})$ and $\alpha$-amylase inhibitory activity $(15.14 \% / \mu \mathrm{g})$ than other fractions. A total of 56 peptide sequences composed of 9-23 amino acid residues per molecule, with a molecular weight of $1.0-2.5 \mathrm{kDa}$, were identified as the most putative antioxidant and antidiabetic peptides using mass spectrometry. The primary structure, amino acid composition and physiochemical properties are important factors in contributing to the bioactivities of cumin seedderived peptides. In conclusion, the extracted bioactive peptides from cumin seeds showed high commercial nutraceutical and pharmaceutical values, which could be incorporated as active ingredients in functional foods or as food supplements.

P70. A workflow on the production of bioactive peptides from food sources based on the case study using pinto beans (phaseolus vulgaris cv. Pinto)

\section{Ngoh YY1* and Gan CY}

Centre for Advanced and Analytical Toxicology Services (CAATS),
Universiti Sains Malaysia, E-mail: ngohyy@hotmail.com

A workflow on the production of bioactive peptides from food sources was discussed in this study with a real case study experiment using Pinto bean (Phaseolus vulgaris cv. Pinto). This workflow proved to be functional and efficient as bioactive peptides derived from Pinto bean protein isolate (PBPI) using Protamex exhibited promising antioxidative and $\alpha$-amylase inhibition activities. Implementation of this workflow is convenient and consuming less time compared to the previous bioactive peptides studies with high possibility of positive outcome being achieved. Generally, this workflow begins with extraction of peptides from protein isolate by carrying out single factors experiments to identify the trend of the extraction parameters. In the pinto bean case study, four extraction parameters were investigated: contact time (0.5-5 h), S/E ratio (10-100), $\mathrm{pH}$ (6.5-9.0) and temperature $\left(30-60{ }^{\circ} \mathrm{C}\right)$. The influential parameters were then studied using a factorial design experiment to determine the most suitable condition in producing peptides with the highest bioactivities. Fractionation using a centrifugal membrane filter with molecular weight cutoffs of 3 $\mathrm{kDa}$ was then performed followed by subjecting into LCMS and MS/ MS analysis using Thermo LTQ/ Orbitrap Velos for the identification of peptide sequences. Peptide Ranker software was applied for the screening of potential bioactive peptides. Both antioxidant and $a$-amylase inhibition activities revealed two peptide sequences each. The sequences found were (PLPPHMLP, ACSNHSPLGWRGH) and (PLPPHDLL, FNPFPSPHTP) for antioxidant and $\alpha$-amylase inhibition activity respectively. The proposed workflow resulting in successful functionality is recommended and applicable in the field of production of bioactive peptides with the hope of exploring and discovering other potential bioactive peptides possessing activities such as antimicrobial, anticancer, antithrombotic, hypocholesterolemic and antihypertensive.

\section{P71. Significance of food composition databases for nutrition agendas}

\section{Machackova $\mathbf{M}^{\star}$}

Institute of Agricultural Economics and Information, Prague, Czech Republic,E-mail:machackova.marie@uzei.cz

Food composition databases (FCDs) are information systems including systematised and organised data concerning the nutrient composition of foods. They have been usually developed on a national level and used by a broad community of users. FCDs are important information source for the sector of nutrition agendas. This contribution covers history of food composition data collection, data sources, availability of FCDs, limits for their use, methods of data documentation, user groups, international cooperation (INFOODS, EuroFIR). A unique interface for simultaneous searches in 28 national food composition datasets - EuroFIR FoodEXplorer - is to be presented. Development of the Czech Food Composition Database www.nutridatabaze.cz will be briefly mentioned.

P72. Nutrition involvement, label reading and eating behaviour

\section{Mulders MDGH ${ }^{1^{*}}$, Klein $\mathrm{O}^{1}$ and Corneille $\mathrm{O}^{1}$}

${ }^{1}$ Université Libre de Bruxelles, Belgium

${ }^{2}$ Université Catholique de Louvain-la-Neuve, Belgium, E-mail: mmulders@ulb.ac.be

Together with the increase of obesity rates, paradoxically, the popularity of healthier foods with lower calorie and fat density has also 
increased. Food intake may be influenced by the perception of a food, like itss perceived healthiness (Paquette, 2005). The judgment of the healthiness of food is oftentimes based on its nutrient content (Carels, Konrad \& Harper, 2006), such as low amount of calories or low fat density, which can be found on the food label. Even if different labeling strategies are used to identify foods, consumers seem confused about what healthy eating should be, or how to use the more technical and numerical information on food labels (Cowburn \& Stockley, 2005). In this research we aim to investigate how label use and label knowledge are influenced by factors like (disordered) eating behavior, numeracy skills and nutrition involvement. We tested the hypothesis that higher nutrition involvement causes better performance on label reading tasks, and examined whether this relation is moderated by numeracy skills. We also tested if disordered eating patterns (measured with the EAT-26) influence nutrition involvement and score on label reading tasks. We compare the scores of people from a healthy population as well as a sample of individuals with a diagnosed eating disorder on several scales such as the Short Numeracy Scale (Lipkus et al., 2001), the Nutrition Involvement Scale (Chandon \& Wansink, 2007), the Newest Vital Sign (Weiss et al., 2005), the Eating Attitude Test-26 (Garner \& Garfinkel, 1979), and an adapted version of the Nutrition Label Survey (Rothman et al., 2006). An initial study showed that numeracy was a moderator of the influence of nutrition involvement on label reading. We are currently collecting additional data and expect to find a positive relation between both EAT-26 and nutrition involvement as well as between nutrition involvement and label reading score.

\section{P73. Dietary trends among Czech children and adolescents between 2002-2014: HBSC study}

\section{Voráčová $\mathrm{J}^{{ }^{*}}$, Sigmund $\mathrm{E}^{1}$, Sigmundová $\mathrm{D}^{1}$ and Rážová $\mathrm{J}^{2}$}

${ }^{1}$ Institute of Active Lifestyle, Faculty of Physical Culture, Palacký University Olomouc, Olomouc, Czech Republic ${ }^{2}$ The National Institute of Public Health, Prague, Czech Republic, E-mail: jaroslava.voracova@yahoo.com

Background: Unhealthy diet in children and adolescents is associated with an early onset of risk factors of chronic diseases that are the leading causes of death globally. Poor dietary patterns also increase the prevalence of obesity in adulthood. Currently, many children skip breakfast, consume soft drinks/sweets and do not eat recommended amount of fruit and vegetables. Poor eating habits in children tend to be carried into adulthood.

Methods: The trends in eating behaviours of Czech 11-, 13- and 15 -year-old children were examined by frequency of breakfast, fruit, vegetable, sweet and soft drink consumption obtained from the Health Behaviour in School-aged Children surveys in 2002, 2006, 2010 and 2014.

Results: Between 2002 and 2014, the findings showed a decrease in daily soft drink $(\mathrm{p}<0.001)$, sweet $(\mathrm{p}<0.01)$ and fruit $(\mathrm{p}<0.01)$ consumption and an increase in daily breakfast eating during weekdays $(p<0.01)$. According to gender variations, significant changes in daily fruit $(\mathrm{p}<0.01)$ and breakfast (during weekdays) $(\mathrm{p}<0.01)$ eating were observed only in girls but not boys. On the contrary, a significant decrease in daily sweet consumption $(\mathrm{p}<0.05)$ was indicated only in boys. Daily vegetable and breakfast at weekend consumption remained stable in both genders over time. More frequent daily fruit, vegetable and breakfast (at weekend) consumption was reported by girls and younger children whereas daily soft drink intake was more prevalent in boys and older children.
Conclusion: There is a need for re-evaluation of current national policies, initiatives and nutritional requirements and regulation in schools to improve eating habits of Czech children. Future research should continue monitoring trends in the Czech Republic to further evaluate effectiveness of on-going changes in national policies and intervention programmes.

\section{P74. Determining the relationship of sleep duration between energy expenditure and food intake}

\section{Deniz $S^{1 *}$ and Saka $\mathbf{M}^{2}$}

${ }^{1}$ Acıbadem Fulya Hospital, Nutrition and Dietetics Department, İstanbul, Turkey

${ }^{2}$ Baskent University, Faculty of Health Sciences, Department of Nutrition and Dietetics, Ankara, Turkey, E-mail: seyma_deniz@ hotmail.com

The current study aimed to determine the relationship of individuals> sleep duration between energy expenditure and food intake. The sample of this study composed of randomly selected individuals who applied to a private obesity clinic in İstanbul, between September and December 2013. Aged between 18-67 years old 7 male and 87 female were participated in the current study. The information about demographic characteristics, nutritional status and sleeping habits were taken via questionnaires. Sleep duration and 3 days food consumptions were recorded by the individuals themselves. Energy expenditure was calculated by using 24 -hours physical activity record form. Life satisfaction was determined by a questionnaire. The average age of participants was $39.2 \pm 11.3$ years, body mass index was $34.3 \pm 6.68$ $\mathrm{kg} / \mathrm{m}^{2}$ and sleep duration was $7.9 \pm 0.99$ hours. When the sleep duration was questioned, it was seen that $20 \%$ of the participants had 6 hours or less, $75.6 \%$ had 7 or 8 hours, and $4.3 \%$ had more than 8 hours of sleep weekdays. On the other hand, $13.8 \%$ of the participants has 6 hours or less, $61.7 \%$ had 7 or 8 hours, and $24.5 \%$ had more than 8 hours of sleep during weekends. When the mean sleep duration of individuals compared energy and fat intake according to week and weekend days, the negative relationship was not found statistically significant ( $p>0.05)$. The difference in basal metabolic rate (BMR), total energy expenditure (TEE), and physical activity levels (PAL) of individuals according to weekend and weekdays mean sleep duration, there was not any statistically significant differences $(\mathrm{p}>0.05)$. Average sleep duration evaluations showed that, there were significant positive correlations between weekend sleep time and BMR and PAL $(r=0.225, r=0.223$ respectively; $\mathrm{p}<0.05$ ). Consequently, sleep is an indispensable part of healthy life. In recent years, the effect of inadequate sleep duration on increasing rates of obesity and diabetes is being remarkable. Many studies indicate the relationship between sleep duration between optimum sleep duration and health.

P75. Comparison of eating disorders status between first and fourth year of nutrition and dietetics students in a private university in Istanbul

\section{Dumlu G*, Bakır B and Karaltı I}

Nutrition and Dietetics Department,Faculty of Health Sciences, Yeditepe University, Turkey, E-mail:gozde.dumlu@yeditepe.edu.tr

Nutrition is balanced and adequate food intake to ensure protection and development of health. When individuals' requirements, habits, family and social environments are evaluated, nutrition is multifactorial issue which shows alterations and diversity according 
to age groups. Nowadays, although healthy eating perceptions are frequently emphasized, "thin body perception" which are affected by social pressure and media" also begins significantly important for last years. This condition which increase in developed societies and some societies influenced by Western culture interest particularly university students. Involvement in new social environment, changing of eating habits, becoming "weight subject" as focus in social media and cultural environment induce development of eating disorders.

When the university students compare according to their department, some studies suggest that nutrition and dietetic students can be in highly risk group for development of eating disorder because of their enhanced knowledge status about "food", "weight control" and "body composition". The aim of this study is determination and comparison of eating disorders status between first and fourth year of nutrition and dietetic students. Two hundred two nutrition and dietetic students were recruited from a private university, in İstanbul. The survey was conducted between September 2013 and 2014. All participation was voluntary and protected by not using names. Anthropometric measurements such as weight $(\mathrm{kg})$ and height $(\mathrm{cm})$ were obtained from all participants and these parameters were used for calculation of body mass index (BMI) and Eating Attitudes Test -40 (EAT-40) was applied for determination of eating disorder status. According to data obtained from this study, while 179 of nutrition and dietetic students $(88.6 \%)$ did not have eating disorder, 6 of students (3\%) have eating disorder and 17 of students (8.4\%) have moderate risk for development of eating disorder. When students are compared according to their class, first year of nutrition and dietetic students exhibited that $3.3 \%$ of them had eating disorder behavior and $9.1 \%$ students had moderate risk for development for eating disorder; fourth year of the students demonstrated that $2.5 \%$ of them had eating disorder behavior and $7.4 \%$ of the students had moderate risk for abnormal eating behavior. The difference between classes and eating disorder behaviors of students was not found statistically significant. $(\mathrm{p}>0.05)$

P76. Evaluation of energy and nutrient content of served and consumed school lunch in an elementary school

\section{Kiran $\mathrm{M}^{1^{*}}$ and Ercan $\mathrm{A}^{2}$}

\section{${ }^{1}$ Merve Kiran Nutrition Counseling Center, Turkey}

${ }^{2}$ Baskent University, Turkey, E-mail: mervekran@gmail.com

Objective: To evaluate the nutrient content of school lunch meals and compare students> average nutrient intake from lunch with the School Meals Initiative (SMI) nutrition standards.

Methods: This study was conducted in a private elementary school located in Zonguldak, Turkey, from January to March 2014. A total of 186 students, aged between 10-15 years old, participated in the study. For 10 consecutive school days the portion sizes of school lunches served was weighed. At the end of every lunch period, each lunch tray was collected and plate wastes were filled into their respective bins separately by their meal components and then were weighed again. The amount of food waste was divided by the number of students attending school lunch and the average plate waste was calculated for energy and nutrients contents. The average daily lunch consumption was computed with the difference between the portion size of lunches served and the average plate waste. The Nutrient Data Base (BEBIS) program was used to evaluate the energy and nutrient intakes of students at school lunch and compared with SMI nutrition standards.

Results: Energy content of school meal only met the requirements of 10-13 year old girls. For the most of the students, protein, total fat, saturated fat, sodium, iron and vitamin A content of meal were more than requirements, while calcium and dietary fiber did not meet the requirements. Only vitamin C content met the SMI standards for all students. Actual consumption of calcium, dietary fiber and vitamin $\mathrm{C}$ did not meet nutritional requirements of all students. However, the students consumed more than requirements for protein, total fat, saturated fat, sodium, iron and vitamin A at lunch.

Conclusions: The school food environment has the potential on childrens> nutritional behaviour. School children spend most of their time in schools where they consume a substantial proportion of their total daily calories. Strategies to improve school nutrition programs, to increase school lunch consumption and to reduce plate waste should be developed.

P77. Association between serum ferritin and hemoglobin levels and bone health in Korean adolescents

\section{Kim $\mathrm{DH}^{1^{*}}$, Jung DW', Park YG ${ }^{2}$, Nam HY1, Nam GE ${ }^{1}$, Han $\mathrm{KD}^{2}$ and} Roh YK $^{3}$

${ }^{1}$ Department of Family Medicine, Korea University, College of Medicine, Seoul, Republic of Korea

${ }^{2}$ Department of Biostatistics, Catholic University, College of Medicine, Seoul, Republic of Korea

${ }^{3}$ Department of Family Medicine, Hallym University, College of Medicine, Chunchon, Republic of Korea,E-mail: kmcfm@hanmail.net

Background: It is important to identify risk factors for low bone mass at a young age. An influence of iron store on bone health in the general population has been reported but has not been studied in adolescents. This study aimed to investigate the relationship between hemoglobin and serum ferritin levels and bone mineral content (BMC) in South Korean adolescents.

Methods: This study was based on data collected during the 2009-2010 Korea National Health and Nutrition Examination Survey. We included 1321 subjects aged 10 to 18 years. BMC was measured at the femur and lumbar spine by dual-energy X-ray absorptiometry, and hemoglobin and serum ferritin levels were examined.

Results: In boys, hemoglobin and serum ferritin levels were positively associated with BMC at total femur and lumbar spine after adjusting for confounders, and hemoglobin levels significantly increased as BMC increased at all sites ( $\mathrm{p}$ for trend $=0.001$ for total femur, 0.01 for femur neck, and $<0.001$ for lumbar spine). Likewise, serum ferritin levels showed increasing trends according to increasing BMC of total femur and lumbar spine in boys ( $\mathrm{p}$ for trend $=0.04$ for total femur.

Conclusions: This study suggests a positive relationship between serum ferritin and hemoglobin levels and BMC in South Korean adolescent boys.

\section{P78. Multiplex immunoline test for food allergens detection}

\section{Smetanová $\mathrm{H}^{{ }^{*}}$, Plicka $\mathrm{J}^{1}$, Rysová $\mathrm{J}^{2}$ and Gabrovská $\mathrm{D}^{3}$}

${ }^{1}$ ELISA development s.r.o., Velké Žernoseky 186, 412001 Litoměřice, Czech Republic

${ }^{2}$ Výzkumný ústav potravinářský Praha, v.v.i., Radiová 7, 10231 Praha 10, Czech Republic

${ }^{3}$ Potravinářská komora ČR, Počernická 96/272, 10803 Praha 


\section{0-Malešice, Czech Republic, E-mail: smetanova@elisadevelopment.cz}

All the allergenic products and substances whose presence in food must be indicated on labelling in accordance with the EU legislation (Regulation EU No 1169/2011). Cereals containing gluten, milk, eggs, nuts, peanuts, soybeans, fish, crustaceans, molluscs, celery, lupin, sesame, mustard and sulphites belong to the group of mandatory declared allergens. The development of a highly sensitive and specific multiplex test able to detect more allergens within a single assay was aim of this work. Multiplex Immunoline test is specific against allergens Arah1 (peanut), beta-lactoglobulin (milk), casein (milk), egg white proteins (egg), tree nuts proteins from hazelnuts, walnuts, almonds, pistachios, Brazil nuts, macadamia nuts and cashew and mustard proteins from Sinapsis alba, Brassica nigra and Brassica juncea. The presence of food allergens can be find out within a short time, which can lead to time savings for food producers and trade in food commodities. Multiplex Immunoline test is based on the binding of antibodies immobilized on the solid phase to an antigens extracted from food samples. The detection is carried out by the biotinylated specific antibodies, streptavidin - alkaline phosphatase and a BCIP/ NBT colorimetrics membrane AP substrate. The different categories of commercial food products were tested for the Immunoline validation and the stability and reproducibility tests were performed.

P79. Comparison of serum vitamin d, calcium and magnesium levels for both type 2 diabetic and non diabetic individuals with nutritional habits

\section{Ozpak Akkus $\mathrm{O}^{\star}$ and Saka $\mathrm{M}$}

\section{Batman State Hospital, Turkey, E-mail: dytozlemozpak@hotmail.com}

This study was carried out to investigate the relationship between type 2 diabetes and serum vitamin $\mathrm{D}$, calcium and magnesium levels by comparing serum vitamin $\mathrm{D}$, calcium and magnesium levels with nutritional habits, anthropometric measurements, physical activity levels and some biochemical findings of both type 2 diabetic and nondiabetic individuals. The study was conducted on patients with type 2 diabetes in the last 5 years, 51 type 2 diabetic patients aged 25 and 45 and 51 healthy individuals. The measurements for both type 2 diabetics and healthy individuals were found to be missing serum vitamin $\mathrm{D}$ (84.3\% and $86.7 \%)$, normal ionized calcium $(82.3 \%$ and $84.3 \%)$ and normal magnesium $(82.3 \%$ and $64.7 \%)$ respectively. The body mass index and waist circumferences for type 2 diabetics (with higher levels of vitamin D, calcium and magnesium) were found to be significantly higher than those of the healthy individuals. A significant positive correlation between serum vitamin $\mathrm{D}$ levels with serum calcium) and the duration of benefiting from sunlight was found $(p<0.05)$. No relationship was found between serum vitamin $\mathrm{D}$, calcium and magnesium levels with HOMA-IR. The daily calcium consumption for $21.6 \%$ of the type 2 diabetics and $25.5 \%$ of the healthy individuals involved in the study were found to be less than $1000 \mathrm{mg}$. The BMI and waist circumferences for type 2 diabetics consuming 1000mg daily calcium. All type 2 diabetic women aged 31-50 take enough amount of magnesium. A significant positive correlation between individuals' magnesium consumption with body weight and serum calcium levels was found. Consequently, there is a need for more randomized controlled studies in order to evaluate the relationship between serum vitamin $\mathrm{D}$ calcium, magnesium and their consumption with type 2 diabetes and insulin resistance.

P80. Effects of bofutsushosan supplements on regulation of lipid and glucose metabolism in a high-fat diet-fed animal model; based on transcriptomic profiles with phenotype characteristics

\section{Kim $\mathrm{YJ}^{{ }^{*}}$, Choi JY ${ }^{2}$, Jung $\mathrm{UJ}^{1}$, Ryu $\mathrm{R}^{1}$, Lee $\mathrm{JH}^{1}$ and Choi MS ${ }^{1,2}$}

${ }^{1}$ Department of Food Science and Nutrition, Kyungpook National University, Daegu, Repulic of Korea

${ }^{2}$ Center for Food and Nutritional Genomics Research, Kyungpook National University, Daegu, Repulic of Korea, E-mail: freewilly59@ hanmail.net

Bofutsushosan (BT), one of Korean herbal medicines, is well known to have beneficial effects on obesity and hypertension. However, changes in transcriptional profiles in response to BT are little known and still needs to be elucidated. In the present study, we investigated the effect of BT on phenotype characteristics and elucidated antiobesity mechanism based on RNA-sequencing analysis transcriptomic profiles in an animal model of obesity. C57BL/6J mice (4-week-old, Male) were fed a normal diet ( $16.58 \% \mathrm{kcal}$ from fat, ND), high-fat diet (60\% kcal from fat, HFD), and HFD supplemented with $1.5 \%(\mathrm{w} / \mathrm{w})$ BT. They were given free access to food and distilled water for 12 weeks. After 12 weeks supplementation of BT, body weight, body weight gain, liver weight and adipose tissue weight were significantly decreased, while muscle weight was significantly increased in BT group. BT not only improved plasma lipid profiles such as total cholesterol and HDL-cholesterol but also remarkably decreased content of hepatic cholesterol and levels of liver damage markers in plasma. BT also led to decreased circulating adipokines and inflammatory cytokines levels. Moreover, BT significantly elevated activities of hepatic lipid- and glucose-regulating enzymes, such as fatty acid synthase, glucose-6phosphate dehydrogenase, glucokinase, and glucose-6-phosphatase. In addition, mRNA sequencing analysis revealed that BT up-regulated genes of adipocytokine- and insulin-signaling pathways in liver. In adipose tissue, BT down-regulated genes involved in lipid metabolism, such as PPAR signaling pathway. These findings indicate that BT can regulate the expression of genes related to lipid and glucose metabolism in a way to control the metabolic disorders occurred during obesity.

\section{P81. Anti-diabetic effects of mate in obese subjects: a} randomized controlled trial

\section{Ryu R $\mathbf{R}^{1^{*}}$, Jung UJ' ${ }^{1}$, Kim $\mathrm{YJ}^{1}$, Jeong TS $^{2}$, Choi MS ${ }^{1,3}$}

${ }^{1}$ Department of of Food Science and Nutrition, Kyungpook National University, Daegu, Republic of Korea

${ }^{2}$ National Research LaboratoryofLipid Metabolism \& Atherosclerosis, Korea Research Institute of Bioscience and Biotechnology, Daejeon, Republic of Korea

${ }^{3}$ Center for Food and Nutritional Genomics Research, Kyungpook National University, Daegu, Republic of Korea, E-mail: sangsang0119@ gmail.com

Mate is well known to have anti-diabetic, anti-inflammatory and anti-obesity properties in animal models. Especially, chlorogenic acid, the main polyphenol of mate, is reported to regulate the activity of enzymes which is associated with glucose metabolism. However, there is little known about its antidiabetic effect in human. In the present study, we investigated the effects of mate ethanol extract (ME) on levels of blood glucose and hormones involved in glucose homeostasis as well as insulin resistance in obese subjects with mild metabolic syndrome. To this study, subjects were randomly assigned to two groups and administered six capsules containing starch ( $3 \mathrm{~g} /$ day, Placebo) and ME 
( $0.426 \mathrm{~g} /$ day) for 12 weeks. Blood samples were collected with heparin to obtain the plasma. After the trial, body weight and body mass index were not significantly changed, while systolic blood pressure was improved in ME group compared to the placebo group. Fasting blood glucose level was significantly decreased in ME group. ME supplement also significantly lowered the baseline-adjusted HbA1c and HOMA-IR levels compared to Placebo group. In addition, the baseline-adjusted plasma GIP level was significantly lowered by ME supplement, although plasma insulin level was not altered. The plasma GOP and GPT levels were not significantly different between the ME supplement group and placebo group. Together, these findings suggest that ME supplementation can reduce risk factors associated with diabetes in obese subjects with mild metabolic syndrome.

P82. Optimization of medium composition for the growth of a potential probiotic strain, lactobacillus salivarius f212, using response surface methodology

Huh $\mathrm{CS}^{1,2 *}$, Park BC ${ }^{1,2}$, Yeo $S Y^{1}$, Lee $S^{1}$

${ }^{1}$ Research Institute of Eco-friendly Livestock Science, Institute of Green-Bio Science and Technology, Korea

${ }^{2}$ Graduate School of International Agricultural Technology, Seoul National University, Korea, E-mail: cshuh@snu.ac.kr

Probiotics in industries should be developed with improvement of the growth activity. The MRS (deMan Rogosa Sharp) medium has been widely used as the commercial medium for lactic acid bacteria. However, MRS medium is not enough to apply to a certain Lactobacillus strain for maximizing its growth because it is comprised of general nutrients which are suitable for the genus Lactobacillus. Therefore, it is important to find optimal growth factors for a strain in industries. These researches have been undertaken with the objective of optimizing media by applying response surface method (RSM). Most of the studies only considered number of viable cells as the response to assess their effects using RSM. However, the difference of our study from reported studies is that we considered two factors; number of viable cells $(\log 10(\mathrm{CFU} / \mathrm{ml}))$ and growth rates (min.). Because the growth rates of bacteria is closely related to bacterial productivity in industry. In this study, we optimized culture medium conditions to enhance the growth ability of a probiotic candidate, Lactobacillus salivarius F212 which was isolated from feces of a weaned pig. At first, we performed a microbiological analysis of L. salivarius F212 strain before optimization of growth medium. In initial screening, carbon and nitrogen sources and growth factors were analyzed by one-factor-at-atime (OFAT) method to confirm their direct influence on the probiotic strain growth. A central composite design was applied to identify the effect on both relative rapid growth rate (min.) and maximum growth number $(\log 10(\mathrm{CFU} / \mathrm{ml}))$. Polynominal regression model with linear and quadratic terms was used for the statistic analysis of the experimental data. Our observations suggest the optimized medium is proper to enhance growth of L. salivarius F212.

P83. Saussurea lappa clarke-derived costunolide preventstnf $\alpha$-induced breast cancer cell migration and invasion byinhibiting $\mathrm{nf}-\kappa \mathrm{b}$ activity

Choi YK ${ }^{\star}$, Woo SM, Kim AJ, Ku JM, Hong SH, Kim JH, Choi HS and Ko SG

Laboratory of Clinical Biology and Pharmacogenomics, Department of Preventive Medicine, Kyung Hee University, Korea, E-mail: goldrain0403@naver.com
Saussurea lappa Clarke (SLC) has been used as a traditional medicine in Korea, China, and Japan for the treatment of abdominal pain and tenesmus. Costunolide, a sesquiterpene lactone isolated from SLC, has diverse medicinal effects. However, the anticancer effects of costunolide are still unclear in breast cancer. In this study, we demonstrate that costunolide suppresses tumor growth and metastases of MDA-MB-231 highly metastatic human breast cancer cells via inhibiting TNF $\alpha$-induced NF- $\kappa \mathrm{B}$ activation. Costunolide inhibited MDA-MB-231 tumor growth and metastases without affecting body weights in the in vivo mouse orthotopic tumor growth assays. In addition, costunolide inhibited in vitro TNF $\alpha$-induced invasion and migration of MDA-MB-231 cells. Costunolide further suppressed TNF $\alpha$-induced NF- $\kappa \mathrm{B}$ signaling activation, resulting in a reduced expression of MMP-9, a wellknown NF- $\kappa \mathrm{B}$-dependent gene to mediate breast cancer cell growth and metastases. Therefore, we conclude that SLC and its derivative costunolide suppress breast cancer growth and metastases by inhibiting TNF $\alpha$-induced NF- $\kappa \mathrm{B}$ activation, suggesting that costunolide as well as SLC may be promising anticancer drugs, especially for metastatic breast cancer.

\section{P84. Sensitizes butein-mediated apoptosis of chronic myeloid} leukemia cells

Woo SM*, Choi YK, Kim AJ, Ku JM, Hong SH, Kim JH and Ko SG

Laboratory of Clinical Biology and Pharmacogenomics, Department of Preventive Medicine, Kyung Hee University, Korea, E-mail: taoya@ hanmail.net

A progression of chronic myeloid leukemia marked by oncogenic $\mathrm{Bcr}-\mathrm{Abl}$ mutation is tightly linked to an alteration of $\mathrm{p} 53$ pathway. It has been known that butein extracted from various plants represses cancer growth. While anti-cancer effects of butein is widely accepted, mechanisms by which butein causes apoptosis of chronic myeloid leukemia cells are yet clearly deciphered. In this study, we demonstrate that butein-induced apoptosis is mediated by p53. KBM5 chronic myeloid leukemia cells expressing wild type p53 were more sensitive to butein than p53-null K562 chronic myeloid leukemia cells in apoptotic cell death. In addition, butein arrested KBM5 cells at $S$ phase and altered expression levels of Cyclins and p53-downstream targets, MDM2 and p21. Moreover, while butein reduced MDM2 protein level in both KBM5 and K562 cells, it resulted in proteasome-independent MDM2 degradation in p53-expressing KBM5 cells but not in p53-null K562 cells. Therefore, our study suggests that p53 sensitizes buteinmediated apoptosis of leukemic cells.

P85. Water extract of deer bones activates macrophages and alleviates neutropenia

Hong $\mathrm{SH}^{\star}$, Ku JM, Choi YK, Woo SM, Kim AJ, Kim JH, Choi HS and Ko SG

Laboratory of Clinical Biology and Pharmacogenomics, Department of Preventive Medicine, Kyung Hee University, Korea, E-mail: znrznr53@naver.com

Extracts from deer bones, called nok-gol in Korean, have long been used to invigorate Qi. While neutropenia is not well detected in normal physiological condition, it could be a cause of severe problems to develop diseases such as infectious and cancerous diseases. Thus, a 
prevention of neutropenia in normal physiology and pathophysiological states is important for maintaining Qi and preventing disease progress. In cell biological aspects, activated macrophages are known to prevent neutropenia. In this study, we demonstrate that water extract of deer bone (herein, NG) prevents neutropenia by activating macrophages. In mouse neutropenia model system in vivo where ICR mice were treated with cyclophosphamide to immunosuppress, an oral administration of NG altered the number of blood cells including lymphocytes, neutrophils, basophils, and eosinophils. This in vivo effect of NG was relevant to that of granulocyte colony stimulating factor (G-CSF) that was known to improve neutropenia. Our in vitro studies further showed that NG treatment increased intracellular reactive oxygen species (ROS) and promoted macrophagic differentiation of mouse monocytic Raw264.7 cells in a dose-dependent manner. In addition, NG enhanced nitric oxide (NO) synthesis and secretions of cytokines including IL-6.

P86. Anti-allergic effects of so-cheong-ryong-tang in ovalbumin-induced allergic rhinitis model

\section{Ku JM*, Hong SH, Choi YK, Woo SW, Kim AJ, Kim JH, Choi HS} and Ko SG

Laboratory of Clinical Biology and Pharmacogenomics, Department of Preventive Medicine, Kyung Hee University, Korea, E-mail: saory ykm@naver.com

Allergic rhinitis (AR) is a global health problem, which is associated with life quality. $40 \%$ of the population worldwide is suffering from AR. Allergic rhinitis (AR) is an allergic inflammation of the nasal airways. The traditional herbal medicine "So-Cheong-Ryong-Tang" is called "Sho-seiryu-to" in Japan and "Xiao-Qing-Long-Tang" in China. So-Cheong-Ryong-Tang (SCRT) contains eight species of medicinal plants. SCRT has been used for the treatment of AR, bronchitis, bronchial asthma, and symptoms for hundreds of years in Asian countries. Recent studies indicated that SCRT inhibits histamine release, degranulation of mast cells, proliferation of eosinophils and basophils and cholinergic effects for nasal gland acinar cells. In the present study, we investigated whether SCRT suppresses the progression of AR in animal model. AR was induced by ovalbumin (OVA). Treatment with SCRT was assessed to study the effect of SCRT on AR in mice. Histological analysis, multiplex cytokine assay, blood analysis, cell viability assay, RT-PCR and Elisa assay were performed to verify inhibitory effect of SCRT on AR. SCRT reduced infiltration of inflammatory cells into nasal cavity. SCRT reduced infiltration of mast cells into nasal mucosa. SCRT reduced the levels of cytokines (IL-4 and LIF) in the serum. SCRT reduced the levels of leukocytes in the blood. SCRT decreased cell viability of HMC-1 cells and splenocyte. SCRT suppressed IL-4 level in HMC-1 cells and splenocyte cells in a dose dependent manner. SCRT suppressed IL- 6 level and TNF- $\alpha$ level in splenocyte. SCRT suppresses the progression of AR induced by OVA. SCRT might be a useful drug for the treatment of AR.

\section{P87. KAJ-001 suppress tumor growth on ct-26 cells by induces cellular senescence}

Kim $\mathrm{AJ}^{\star}$, Choi YK, Woo SM, Hong SH, Ku JM, Kim JH, Choi HS and Ko SG

Laboratory of Clinical Biology and Pharmacogenomics, Department of Preventive Medicine, Kyung Hee University, Korea, E-mail: kitkim819@naver.com

Cellular senescence is a state of irreversible stimuli for inhibition of somatic cells growth. Senescence caused by various factors of stress, such as telomeres shortening, DNA damage, oxidative stress or stimulus to drugs. Senescent cells exhibit changes, including an enlarged and flatted phenotype, expression of p16, p53 and p21. Especially, p16 is one of the most used senescence marker that expression increase with during senescence. Recently, senescence is considered to be an anticancer mechanism, which arrest the uncontrolled proliferation in many cancer cells. In this study, we demonstrated that KAJ-001, new herbal formula induces cellular senescence on CT-26 colon cancer cells. KAJ-001 inhibited tumor growth without loss of body weight in Balb/c mice. KAJ-001 effects on cell viability but does not occur apoptosis. Furthermore, KAJ-001 induces expression of p16 in CT-26 cells by p38 mitogen-activated protein kinase pathway. Therefore, we conclude that KAJ-001 suppress tumor growth on CT-26 cells by induces p16 upregulated-cellular senescence.

\section{P88. Validation of biomarkers of oxidative stress in large-} scale human studies

Jansen $\mathrm{E}^{\mathbf{1}^{\star}}$, Beekhof $\mathrm{P}^{1}$, Viezeliene $\mathrm{D}^{2}$, Ruskovska $\mathrm{T}^{3}$, Muzakova $\mathrm{V}^{4}$ and Skalicky $\mathbf{J}^{5}$

${ }^{1}$ National Institute for Public Health and the Environment, Bilthoven, Netherlands

${ }^{2}$ Lithuanian University of Health Sciences, Kaunas, Lithuania

${ }^{3}$ Goce Delcev University, Stip, Macedonia

${ }^{4}$ University of Pardubice, Czech Republic

${ }^{5}$ Regional Hospital of Pardubice, Czech Republic, E-mail: eugene. jansen@rivm.nl

Objectives: Oxidative stress has been proposed to be important in age-related processes and chronic diseases. Before biomarkers of oxidative stress are measured in large-scale human studies, they need validation on storage stability and other characteristics. Amongst several candidates (MDA, FRAP, TAS, TOS, GSH, GPX, SOD), three biomarkers were selected which cover different aspects of the oxidative stress process.

Methodology: ROM (Reactive Oxygen Metabolites, Diacron) was used as biomarker of oxidative stress, BAP (Biological Antioxidant Potential, Diacron) for anti-oxidant status and TTL (Total Thiol Levels, RelAssay) for the redox status (DX, Beckman-Coulter).

Results: The assays for the biomarkers ROM, BAP and TTL were adapted for an auto-analyzer (DX, Beckman-Coulter) with very good reproducibility. The biomarkers were stable on short- and long-term storage. Also they showed no significant differences in blood samplings at different times of the day, nor a post-prandial effect. This set of biomarkers was successfully applied in several large-scale European studies (total 20,000 samples) on aging and nutrition.

Conclusion: A set of three biomarkers of oxidative stress was selected for use on an auto-analyzer and applied successfully in largescale European studies.

P89. Aluminium, cadmium and nickel induce oxidative stress by the iron metabolism

Viezeliene $\mathrm{D}^{1^{*}}$, Jansen $\mathrm{E}^{2}$, Beekhof $\mathrm{P}^{2}$, Gremmer $\mathrm{E}^{2}$, Sadauskiene $\mathrm{I}^{1}$ and Ivanov $L^{1}$

${ }^{1}$ Lithuanian University of Health Sciences, Kaunas, Lithuania 
${ }^{2}$ National Institute for Public Health and the Environment, Bilthoven, the Netherlands, E-mail: daleviezeliene@lsmuni.lt

Objectives: Aluminium ( $\mathrm{Al})$, cadmium (Cd) and nickel (Ni) are abundant environmental metals and possible contributors to neurotoxicity, hepatotoxicity or nephrotoxicity. In the present study, effects of these metals on several biomarkers in serum, liver, kidney, and brain tissues of mice were studied.

Methodology: Balb/c mice were exposed to $\mathrm{Al}, \mathrm{Cd}$ and Ni by i.p. injection daily during 14 days. Oxidative stress (ROM), total thiols, biochemical markers and cytokines were measured in the blood and tissue homogenates.

Results: An increase of oxidative stress (ROM) and a decrease in the redox status (total thiols) was observed in the blood. The iron metabolism (total iron and iron saturation of transferrin) was disturbed by $\mathrm{Al}$ and $\mathrm{Cd}$, but not by $\mathrm{Ni}$. All three metals affected the liver and kidney (not brain) as was shown by induction of tissue enzymes, inflammatory cytokines and disturbance of redox status.

Conclusion: Mice exposure to $\mathrm{Al}$ and $\mathrm{Cd}$ resulted in a disturbance of the iron metabolism with a release of iron from its binding protein transferrin. As a result, the liberated free iron caused oxidative stress reactions in serum. Ni did not displace iron, but show a similar toxic profile in the tissues as for $\mathrm{Al}$ and $\mathrm{Cd}$.

P90. Best practices on chondroitin quality in a globalized supply chain: quality regimes, standards, and methodologies

Hildreth $\mathrm{J}^{{ }^{*}}$, Volpi $\mathrm{N}^{2}$, Zhang $\mathrm{W}^{1}$, Dinh $\mathrm{H}^{3}$ and Oketch-Rabah $\mathrm{H}^{3}$

${ }^{1}$ Synutra Pure, Ltd., Rockville, MD, USA

${ }^{2}$ University of Modena, Modena, Italy

${ }^{3}$ United States Pharmacopeia, Rockville, MD, USA, E-mail: jhildreth@synutrapure.com

There is modest clinical evidence supporting the use of Chondroitin Sulfate (CS) for pain management in arthritis patients. In the United States CS is used as a dietary ingredient in dietary supplements intended to support joint health, while in the European Union countries CS is included in treatment regimens for osteoarthritis pain management. Accordingly the United States Pharmacopeia has set CS quality standard for dietary supplement grade CS, while the European Pharmacopoeia's quality standard are for pharmaceutical grade chondroitin sulfate. It has been reported that specific activity of CS depends on the quality: such as chondroitin sulfate structure and properties that determine its bioavailability and efficacy. Thus CS quality affects therapeutic utility of commercial preparations, particularly products manufactured to meet pharmaceutical standards versus products manufactured to meet food grade standards (dietary supplements). In this poster we discuss various testing methods available that can be used to establish identity, purity, strength, composition and limit of contaminants of CS. To ensure high quality and purity of CS material, we recommend the use of compendial CS standards with complementary analytical procedures as well as adherence to proper quality systems, good laboratory practices and training. These practices will ensure that only high quality CS is used in medicines and dietary supplements so that patients and consumers can benefit appropriately.
P91. A high protein diet and isometric exercises improve body composition in er+ breast cancer patients treated with antiestrogenic medication

\section{Artene $\mathrm{D}^{1^{*}}$, Blidaru $\mathrm{A}^{2}$ andBordea $\mathrm{CI}^{3}$}

${ }^{1}$ Carol Davila Medicine University, Bucharest, Romania

${ }^{2}$ Department of Surgery at Carol Davila Medicine University

${ }^{3}$ Oncology Department at Alexandru Trestioreanu Institute of Oncology, Bucharest, Romania, E-mail: artene.diana@yahoo.com

Manybreast cancer patients gain weight during treatment increasing recurrence, oncology specific mortality and general mortality risks. Breast cancer diagnosis and treatment overthrow patients' lifestyle aggravating sedentariness and any preexisting weight gain causes like insulin and leptin resistance, dysbiosis and dyslipidemia. The aim of this study is to evaluate the efficiency of a high protein diet - based on foods naturally high in proteins, omega-3 fatty acids, calcium, probiotics and prebiotics - and of an isometric exercise interventions to generate fat loss without muscle loss in ER+ breast cancer patients taking antiestrogenic treatment. We randomized $50 \mathrm{ER}+$ breast cancer patients - taking either Tamoxifen or Aromatase Inhibitors (AI) after surgery and chemotherapy - to follow the high protein diet, or the diet and 4 ' isometric exercises per day for 8 weeks. Patients were instructed to eat only when hungry and to keep a food journal. We measured weight and body composition with a bioelectrical impedance scale after checking for hydration status. The diet group lost $2.17 \pm 2.42 \%$ body fat $(\mathrm{p}=0,000)$ with no muscle loss, and there was no statistical difference between patients taking Tamoxifen or AI regarding body composition evolution. The diet + isometric exercise group lost 2.2 more pounds than the diet only group and $0.66 \pm 0.91 \%$ visceral fat $(\mathrm{p}=0,001)$ also with no muscle loss. AI patients from the diet and exercise group did not improve muscle mass - maybe because of the musculoskeletal impact of AI medication.

In conclusion, a high protein diet can decrease body fat in ER+ breast cancer patients on antiestrogenic medication. Adding a daily minimal exercise protocol to a high protein diet decreases visceral fat which is more hormonally active. And resistance-training exercises are more appropriate for patients on AI.

\section{P92. Anti-escherichia coli activity and anti-mutagenic activity of phoenix dactylifera seeds}

\section{Gülden $\mathrm{G}^{\star}$, Elif AE and İbrahim HE}

Department of Food Engineering, University ofMersin, 33343, Mersin, Turkey, E-mail: gulgok33@gmail.com

In the past few years there have been attempts to use agricultural by-products for potential health benefits in the diet formulation of human nutrition.For Muslims all over the world dates are used as food about 6000 years and are mentioned importantly in many times in the Quran.The date seeds have possed extractible high value components for safely human consumption as functional foods and ingredients in nutraceuticals. Date seeds represent about $10-15 \%$ of the date fruits weight and have phytochemical compositions and good nutritional values. However, date seeds are utililized as domesticated animal feed and waste and have very little and inadequate use at present. Date seeds have been scientifically demonstrated to have therapeutic implications against such as diabetes, hyperlipidemia, obesity, cholesterol, coloncancer and intestinaldisordersdue to high dietary fiber contents and bioactive constituents. Additionly date seeds have antiviral, 
gastrointestinal protective, hepatoprotective and nephroprotective activities at preclinical studies. The seeds of date include high amounts of bioactivecompounds (polyphenolic antioxidants and dietary fiber), which may have positive health effects and contribute to preventing various infectious diseases. In the study, the anti-Escherichia coli activity and anti-mutagenic activity of date seeds water extract from date palm three cultivars grown (Safawi, Sughai, Mabrum) in Madinah from Saudi Arabia were assessed by discdiffusionmethod and Umu-test using S.typhimuriumTA1535/pSK1002, respectively. The MIC values of date fiber extracts on E. coli were determined 0.26, 0.51, $1.03 \mathrm{mg} /$ $\mathrm{mL}$ for Safawi, Sughai and Mabrum, respectively. Acording to Umutest, Mabrum, Safawi and Sughai were highly suppressed $67.6 \%, 56.6 \%$, $14.4 \%$ of the mutagenicity of furylfuramide(S9-) and 3.6\%, $43.1 \%$, $16.5 \%$ of the mutagenicity of 2 -aminoanthracene $(\mathrm{S} 9+)$, respectively.

P93. Establishment of novel microbial criteria by monitoring of indicator organisms and food-borne pathogens in various food categories

\section{Heo EJ ${ }^{\star}$, Kim SH, JoonIS, Kim YH, Song BR, Kim JK and Kwak HS}

Food Microbiology Division, Ministry of Food and Drug Safety, Korea, E-mail:dvmheo@korea.kr

The food safety regulation and standard could be varying from country to country and could cause issues in trading unless the regulation is grounded by scientific knowledge.

In this research, we monitored the microbial population and analyzed the results to determine the actual level of microbial contamination in various foods using relatively new statistical analysis (Microbiological sampling plan, International Commission on Microbiological Specification for Foods). Our goal is building entirely new standards for various food categories addressed in the Korean "Food Code". Indicator organisms (i.e. total aerobic count, E.coli and coliform) and food-borne pathogens (i.e. Bacillus cerus, Vibrio parahaemolyticus, Staphylococcus aureus and Clostridium perfringens) have been monitored in forty four food categories. As the result of the monitoring, total aerobic count, E. coli and coliform in noodle were found at the range of $0 \sim 5.70$ and $0 \sim 3.80 \log 10$ CFU/g, respectively. Long shelf-life foods such as coffee and soft drink did not show any contaminations of those indicators except total aerobic count with contamination range of $0 \sim 1.30 \log 10 \mathrm{CFU} / \mathrm{g}$ and $0 \sim 0.30 \log 10 \mathrm{CFU} / \mathrm{g}$, respectively. Fermented foods such as Kimchi and Korean traditional sauces showed B. cereus contamination of $0 \sim 2.10 \log 10 \mathrm{CFU} / \mathrm{g}$ and $0 \sim 3.30 \log 10 \mathrm{CFU} / \mathrm{g}$, respectively. With the monitoring, we proposed revised version of statistic standard for microbial contaminations in 6 different food categories. We also proposed new regulations for 2 types of foods including Kimch and alcohol. The proposed standards here will be great addition in the «Food Code» after evaluation of social and economical impact as well as professional advisory committee discussion.

P94. Development of novel real-time pcr assay for the detection of toxoplasma gondii in retail meat products

Heo $\mathrm{EJ}^{{ }^{*}}$, Suh $\mathrm{SH}^{1}$, Joo $\mathrm{IS}^{1}$, $\mathrm{Kim} \mathrm{SH}^{2}$, Yoon $\mathrm{SH}^{2}$, Lee $\mathrm{JS}^{2}$ and Kwak HS

${ }^{1}$ Food Microbiology Division, Ministry of Food and Drug Safety, Cheongju-si, Chungcheongbuk-do, Korea

${ }^{2}$ Microbiology Division, Ministry of Food and Drug Safety, Cheongjusi, Chungcheongbuk-do, Korea, E-mail:dvmheo@korea.kr

Although reasonable numbers of PCR amplification assays were developed, the majority of rapid detection of Toxoplasma gondii (T. gondii) in animals and their meat products has been done by immunogenic assays. Thus, there is still a need for more reliable and certified PCR amplification method for T. gondii detection in retail meat products. Recently, a 529-bp repeat element that exists in 200-300 copies per genome of $\mathrm{T}$. gondii genome has been spotlighted for its usefulness as potential detection sites. In this study, three amplification sites in 529-bp repeat element were selected for the detection of three types of T. gondii (type I, II and III) used for the development of real-time PCR assay. Each primer sets were targeted 80-bp (P1), 82bp (P2) and 70-bp (P3) of different regions of 529-bp element. The First and third primer sets (P1 and $\mathrm{P} 3$ ) had shown strong positivity with all three types of $\mathrm{T}$. gondii while the second primer set (P2) had not shown any amplification. The specificity test performed with 14 different food-borne pathogens as well as 3 protozoan parasites such as Giardia lamblia, Ctyptosporidium parvum and Entamoeba histolytica. Addition of an internal amplification control did not affect the PCR performance and was useful to monitor PCR inhibition. The real-time PCR developed in this study shown promising results to be used in $\mathrm{T}$. gondii detection in retail meat samples.

P95. Streptomyces as antagonist of mycotoxigenic fungi in cereals

\section{Meklat $\mathrm{A}^{1,2^{*},}$ Bouras $\mathrm{N}^{1,3,}$ Mathieu $\mathrm{F}^{4}$ and Sabaou $\mathrm{N}^{1}$}

${ }^{1}$ Laboratoire de Biologie des Systèmes Microbiens (LBSM), Ecole Normale Supérieure de Kouba, Alger, Algeria

${ }^{2}$ Département de Biologie et Physiologie Cellulaire, Faculté des Sciences de la Nature et de la Vie, Université Saâd Dahleb, Blida, Algeria

${ }^{3}$ Département de Biologie, Faculté des Sciences de la Nature et de la Vie et Sciences de la Terre, Université de Ghardaïa, BP 455, Ghardaïa 47000, Algeria

${ }^{4}$ Laboratoire de Ge'nie Chimique UMR 5503 (CNRS/INPT/ UPS),INP/ENSAT de Toulouse, Université de Toulouse, 1 Avenue de l'Agrobiopôle, B.P. 32607, 31326 Castanet-Tolosan Cedex, France, E-mail:atika_mek@yahoo.fr

The goal of this work is to study the antagonistic properties of newly isolated strain of Streptomyces (S3) against different mycotoxigenic fungal species belonging to Aspergillus, Penicillium and Fusarium. The obtained results showed that the strain is active against all targeted fungi. Furthermore, the experiment was performed by testing three culture media, and by evaluating the production of the antifungal compounds (measured by the agar well diffusion method) during kinetics in shaken cultures. The culture medium Bennett was chosen to enhance the production of antifungal compounds by the used strain of Streptomyces. The comparison of antibiography results indicated that the Bennett medium is better for the production of the antifungal activity produced by the strain S3. The UV-visible spectroscopy indicated the non-polyenic nature of the produced antifungal compounds. The purification by HPLC showed that the strain S3 produced different antifungal compounds.

P96. White sorghum flour characterization used to prepare dietetic bread and cookies

\section{Kadri $\mathrm{F}^{1^{*}}$, Boujeniba $\mathrm{M}^{1}$ and Mokrane $\mathrm{H}^{2}$}

${ }^{1}$ Department of Natural Sciences, Ecole Normale Superieure de Kouba, Algeria 
${ }^{2}$ Department of of Chimestry, Laboratory of Bioactif Products and Biomass Valorization, Ecole Normale Superieure de Kouba, Algeria, E-mail:fkadri74@hotmail.com

Sorghum is a gluten-free cereal that is a safe alternative for people with celiac disease and gluten intolerance. It also presents a variety of other health benefits. The objective of this work is to value local cultivars of sorghum in the human diet. Composite flour of soft wheat and white sorghum were evaluated for their technological and organoleptic properties for the preparation of dietetic bread and biscuits. Flours showed compliance with standards for the water content which varies between $9.94 \%$ and $12.47 \%$, and fat acidity ranging between $0.029 \%$ and $0.087 \%$. Sorghum flour presented a high level of ash $(1.79 \%$ Dry Matter) which enabled the enrichment of composite flours. Technological analyzes showed that the falling number increased proportionally with the incorporation rate of sorghum flour, from 242 up to 314 seconds. Gluten rate decreased from $24.18 \%$ to 0 for the wet gluten and from $8.47 \%$ to 0 for the dry gluten according to the incorporation rate of sorghum flour. The alveogram showed that wheat flour incorporated at $25 \%$ of sorghum flour retained the properties of flours intended for panification. Composite flour at $25 \%$ sorghum yielded highly appreciated bread by $80 \%$ of tasters. However, the composite $50 \%$ sorghum flour gave a biscuit enjoyed by a majority $(80 \%)$ of tasters, for its taste and crunchy texture. The $100 \%$ sorghum flour was appreciated by $60 \%$ of tasters for its sandy texture, taste and smell of grain. It is interesting to popularize the use of sorghum flour alone or when incorporated to wheat flour in order to take advantage of its nutritional and dietary values.

\section{P97. Determination of anthropometric measurements of turkish women by age groups}

\section{Mendane $S^{\star}$, Sinem M, Esra K, Perim T and Gül K}

Department of Nutrition and Dietetics, Health Science Faculty, Başkent University, Turkey, E-mail: saka@baskent.edu.tr

The prevalence of obesity has increased dramatically in populations of developed countries and has become a major challenge to public health. Anthropometric measurements represent an important component of nutritional assessment in women. The aim of this study was to determine the relationship between anthropometric measurements and age groups. A total 638 volunteer women (age between 20-63 years) participated in this study. A questionnaire consisted of anthropometric measurements (body weight, height, waist circumference, waist-to-height ratio and neck circumference) and demographic features were administered by face to face interview. Body Mass Index was calculated. Body Mass Index and waist circumference were evaluated according to World Health Organization classifications. All data was evaluated by SPSS Windows 17.0 for Windows. The mean age of the participants was $35.1 \pm 12.10$ years. The $40.0 \%$ of women were in $20-29,22.9 \%$ in $30-39,21.9 \%$ in $40-49,15.2 \%$ in 50 years and over age group. The mean body weight, Body Mass Index, waist, hip, waist/ hip ratio, waist/height ratio, neck and mid upper arm circumferences increase with age. According to Body Mass Index ( $\geq 25 \mathrm{~kg} / \mathrm{m} 2)$, waist circumference $(\geq 88 \mathrm{~cm})$ and waist/height ratio $(\geq 0.5)$; the obesity prevalence in women aged 50 years and over were $52.6 \%, 73.2 \%, 62.9 \%$ , respectively. In women, Body Mass Index correlated positively with waist/height ratio $(r=0.901, p=0.000)$, body weight $(r=0.940, p=0.000)$, mid upper arm circumference $(\mathrm{r}=0.637, \mathrm{p}=0.000)$, waist circumference $(\mathrm{r}=0.913, \mathrm{p}=0.000)$, neck circumference $(\mathrm{r}=0.649, \mathrm{p}=0.000)$ and waist/ hip ratio $(\mathrm{r}=0.517, \mathrm{p}=0.000)$. As a conclusion the evaluation of obesity, BMI, waist, hip, neck circumferences, waist/hip and waist/height ratios are valuable assessments in epidemiological studies and clinical practice.

P98. Obesity and nutritional status among children with autism

\section{Mendane $S^{\star}$, Gül K and Sinem M}

Department of Nutrition and Dietetics, Health Science Faculty, Başkent University, Turkey, E-mail: saka@baskent.edu.tr

Autistic children are often described as having unusual eating habits and physically inactive. The aim of this study was to evaluate the body mass index and nutritional status of children with autism. A total of 94 autistic children (59 male, 35 female) with an age between 5-18 years participated in this study. A questionnaire was administered by face to face interview. Body weight and height were measured by dietitians. Body Mass Index (BMI) was calculated [body weight $(\mathrm{kg}) /$ height (m)2] and categorized according to World Health Organization classifications. Nutritional status of the children was evaluated by food frequency questionnaire. SPSS 13.0 for Windows was used to evaluate all data. The mean BMI of the male and female autistic children was $21.1 \pm 5.22 \mathrm{~kg} / \mathrm{m} 2$ and $20.8 \pm 6.59 \mathrm{~kg} / \mathrm{m} 2$, respectively ( $p>0.05)$. The overweight and obese percentages of the male and female autistic children were $23.7 \%, 42.4 \%$ and $17.1 \%, 31.4 \%$, respectively. There were no statistically significant differences by gender $(\mathrm{p}>0.05)$. According to autism degree, overweight and obesity is more prevalent in mild group than moderate and severe groups, but the differences were not statistically significant $(\mathrm{p}>0.05)$. The mean daily dietary energy, protein (total energy \%), carbohydrate (total energy \%) and total fat (total energy \%) intakes of the male and female autistic children were $1565 \pm 507.51 \mathrm{kcal}, 15.4 \pm 3.10 \%, 46.6 \pm 8.57 \% 37.9 \pm 7.06$ $\%$ and $1359 \pm 574.51 \mathrm{kcal}, 15.4 \pm 4.37 \%, 47.3 \pm 10.53 \%, 37.1 \pm 8.96 \%$, respectively. There were no significant differences between males and females $(p>0.05)$. As a conclusion, obesity frequency and dietary total fat intake are higher than the recommendations in autistic children. Researches are needed to determine the risk factors associated with obesity in children with autism.

\section{P99. Evaluation of nutritional status, biochemical parameters and anthropometric measurements of patients with non- alcoholic fatty liver disease}

\section{Kiziltan $G^{*}$ and Tekin $P$}

Department of Nutrition and Dietetics, Health Science Faculty, Baskent University, Ankara, Turkey, E-mail: gkizilta@baskent.edu.tr

The aim of this study was to determine the nutritional status, biochemical parameters and anthropometric measurements of patients with non-alcoholic fatty liver disease. The research was conducted on 100 (63 women, 37 men) new diagnosed patients with fatty liver at Baskent University Ankara Hospital Internal Diseases Gastroenterology Department. A questionnaire, consist of demographic features, general and nutritional habits was administered by a dietitian. Anthropometric measurements and body composition were evaluated; body mass index was calculated. Nutritional status was determined by food frequency questionnaire. All data was evaluated by SPSS 17.0 for Windows. The mean age of the patients was $44.68 \pm 13.58$ years. The most frequent complications among patients were insulin resistance, obesity, hyperlipidemia and hypertension. The $54.1 \%$ of men and $66.7 \%$ of women were obese. The mean serum levels of fasting blood glucose, ALT, total cholesterol, triglyceride, LDL-cholesterol and uric acid were higher than reference ranges while serum HDL-cholesterol levels were 
lower than the references. The $83.1 \%$ of the patients' HOMA-IR value was found 2.5 and over. It was determined that $54.1 \%$ of men and $44.4 \%$ of women skipped at least one meal. Daily total energy and fat intake was higher than recommendations among patients $(\mathrm{p}<0.05)$. It was found that $61 \%$ of the patients craved sugar and sugary foods and the most craved sugary foods were desserts. The $21 \%$ of the patients' daily fructose intakes were $50 \mathrm{~g}$ and above, the $65 \%$ of the patients' dietary sucrose intakes were higher than the recommendations. The total daily energy intakes of both men and women were lower than their daily energy expenditure $(\mathrm{p}<0.05)$. As a result, it was determined that factors such as high fructose consumption, high-fat diet, high energy intake, low energy consumption, irregular meals, insulin resistance, obesity and hyperlipidemia are triggering risk factors for Non-Alcoholic Fatty Liver Disease.

P100. The effect of medical nutritional theraphy on metabolic syndrome components in patients with hyperthyroidism and hypothyroidism

\section{Kiziltan $\mathrm{G}^{\mathbf{1}^{*}}$ and Dirikli Bardak $\mathrm{N}^{2}$}

${ }^{1}$ Department of Nutrition and Dietetics, Health Science Faculty,Baskent University, Ankara, Turkey

${ }^{2}$ Nazal Nutrition Center, Nicosia, Northern Cyprus, E-mail: gkizilta@baskent.edu.tr

The objective of this study was to determine the effect of medical nutritional therapy on metabolic syndrome components in patients with hyperthyroidism and hypothyroidism. The study was carried on 120 women ages between 20-64 years between August 2014 and October 2014. During 3 month periods an individualized medical nutritional therapy was applied to the patients. To analyze the nutritional status of the patient, food frequency questionnaire and three days food records were administered. Some anthropometric measurements and biochemical parameters were analyzed and physical activity status were evaluated. The metabolic syndrome was determined by Adult Treatment Panel III (ATP III) criteria. The mean age of the patients was $43.4 \pm 11.36$ years. The percentages of the patients with hypothyroidism and hyperthyroidism were $84.6 \%$ and $15.4 \%$, respectively. The mean body mass index (BMI) of the patients with hypothyroidism was $30.44 \pm 5.67 \mathrm{~kg} / \mathrm{m}^{2}$ at the beginning of the study, $28.19 \pm 5.34 \mathrm{~kg} / \mathrm{m}^{2}$ at the end of the study. In hyperthyroidism group the BMI at the beginning was $24.06 \pm 2.73 \mathrm{~kg} / \mathrm{m}^{2}$ whereas at the end of the study the value was decreased to $22.69 \pm 2.54 \mathrm{~kg} / \mathrm{m}^{2}$. The changes of BMI in patients with hyperthyroidism and hypothyroidism were statistically significant $(\mathrm{p}<0.05)$. According to ATP III criteria the metabolic syndrome prevalence at the beginning of the study in patients with hypothyroidism and hyperthyroidism were $75.2 \%$ and $15.8 \%$, respectively. At the end of the study the metabolic syndrome prevalence was decreased in both patients with hypothyroidism and hyperthyroidism dramatically $(\mathrm{p}<0.05)$.There were positive correlations between the serum TSH (thyroid stimulated hormone) and BMI $(\mathrm{r}=0.292, \mathrm{p}<0.05)$; insulin resistance (HOMA-IR) and BMI $(\mathrm{r}=0.639, \mathrm{p}<0.05)$.

P101. The evaluation of anthropometric measurements and body images of young adults attended to a private sport center in Turkey

\section{Turker $\mathrm{PF}^{1^{*}}$ and Avan $\mathrm{Z}^{2}$}

${ }^{1}$ Department of Nutrition and Dietetics, Baskent University, Health Science Faculty, Turkey

\section{${ }^{2}$ Ankara Community Sport Center, Turkey, E-mail: pfturker@} baskent.edu.tr

The aim of this study was to evaluate anthropometric measurements, body image of individuals attended to a private sport center in Turkey. The study was conducted on 100 individuals, \%45 of whom are female and $\% 55$ of whom are male, between the ages of 18-30 years. A questionnaire form was administered including the questions related with sociodemographic attributes and nutritional habits by face to face interview method. Some anthropometric measurements of individuals were assessed. Body mass index (BMI) was evaluated by World Health Organisation standards. "Berscheid, Walster and Bohrnstedt Body Image Questionnaire" and "Fox Body Attractiveness Subscale of Physical Self-Perception Profile'(PSPP)" scales were used to determine body image satisfaction and the perceived body attractiveness, respectively. The mean age of individuals was $26.93 \pm 2.55$ years. The mean BMI of the male and female participants were $26.33 \pm 4.16 \mathrm{~kg} / \mathrm{m} 2$ and $23.42 \pm 5.47 \mathrm{~kg} / \mathrm{m} 2$, respectively. The mean of waist/hip ratio individuals of the male and female were $0.86 \pm 0.05$, $0.73 \pm 0.08$, respectively. When body image satisfaction were examined according to gender, body image satisfaction score of males $(3.90 \pm 0.57)$ were considerably higher than females $(3.60 \pm 0.64)(\mathrm{p}<0.05)$. On the other hand, analysis indicated no significant differences the perceived body attractiveness ( $p>0.05$ ). When analyzing body image satisfaction of female and male individuals according to BMI, mean body image satisfaction score were significantly lower in obese males (3.46 \pm 0.64$)$ compared to normal weight $(4.00 \pm 0.48)$ and overweight males $(4.01 \pm 0.57)(\mathrm{p}<0.05)$. It was found that body attractiveness score in obese male and female individuals was lower than normal weight and overweight participants. It was also determined that underweight female individuals' body attractiveness score was the highest compared with the others. However, females whose waist hip/ratio higher than 0.85 more satisfied with their body image than whose waist/hip ratio under $0.85(\mathrm{p}<0.05)$. As a conclusion, anthropometric measurements play an important role in body image and body attractiveness satisfactions.

\section{P102. In vitro screening for anti-dementia activity of seaweed extracts}

\section{Son $\mathrm{H}^{\star}$, Um M, Yoon M, Cho S, Kim I, Han D and Lee C}

Divisions of Functional Food Research, Korea Food Research Institute, Seongnam 463-746, Republic of Korea, E-mail: Son.Hyuniung@kfri.re.kr

Alzheimer's disease (AD) is the most common form of dementia in the elderly. Considering the complex multifactorial etiology of $\mathrm{AD}$, drugs such as donepezil, tacrine, and galantamine were developed; however, the potentially dangerous side effects of these drugs has limited their use. Thus, current study is investigating whether phytochemicals in plant and food exert beneficial effects on prevention and treatment of $\mathrm{AD}$. In present study, we investigated that methanolic extracts of 15 kinds of seaweed from Jeju Island (South Korea) were screened for acetylcholinesterase and $\beta$-secretase inhibitory activity, antioxidant activity, and neuronal survival in order to evaluate their potential as anti-dementia agent. As a result, Ecklonia cava, Hydroclathrus clathratus, and Ecklonia kurome extracts were found to be the most effective on acetylcholinesterase inhibitory activity. $\beta$-secretase is the key enzymes in amyloid precursor protein processing. In $\beta$-secretase activity assay, Ecklonia kurome extract was effectively inhibited in a dose-dependent manner $(\mathrm{p}<0.05)$. The antioxidant activity of seaweed extracts was measured by using 2,2 -azino-bis-3-ethylbenzothiazoline- 
6-sulphonic acid (ABTS) assay. It is found that Ecklonia kurome extracts had the highest ABTS scavenging activity (IC50 $=0.07 \pm 0.01$ $\mathrm{mg} / \mathrm{ml}$ ). To determine if seaweed extracts can protect cultured human neuronal cells from $\beta$-amyloid-induced neuronal cell death, we treated SH-SY5Y cells with $\beta$-amyloid(1-42). As expected, the neuronal cell death induced by $\beta$-amyloid in SH-SY5Y cells was diminished by the Grateloupia lanceolata, Sargassum siliquastrum, and Ecklonia kurome extracts. Taken together, these results showed that the Ecklonia kurome extract has the potential anti-dementia activity, which suggests that it might provide an effective strategy for improving dementia.

\section{P103. Image analysis on adipocytes for clinical diagnose on} breast cancer

Suárez-Nájera $\mathrm{LE}^{1^{*}}$, Chanona-Pérez $\mathrm{JJ}^{1}$, Valdivia-Flores $\mathrm{A}^{2}$, MéndezMéndez $\mathrm{JV}^{3}$, Salcedo-Vargas $\mathrm{M}^{4}$ and Cárdenas-Pérez $\mathrm{S}^{1}$

${ }^{1}$ Departamento de Ingeniería Bioquímica, Instituto Politécnico Nacional, Escuela Nacional de Ciencias Biológicas, Plan de Ayala y Carpio s/n, Santo Tomás, C.P. 11340, México, D.F.

${ }^{2}$ Instituto Politécnico Nacional, Centro Interdisciplinario de Ciencias de la Salud, Unidad Milpa Alta, Carr. Xochimilco-Oaxtepec Km 39.5, C.P. 12000, México, D.F.

${ }^{3}$ Centro de Nanociencias y Micro y Nanotecnologías, Instituto Politécnico Nacional, Unidad Profesional “Adolfo López Mateos", Luis Enrique Erro s/n, Zacatenco, C.P. 07738, México, D.F.

${ }^{4}$ Centro Médico Nacional Siglo XXI, Hospital de Oncología, Laboratorio de Oncología Genómica, Av. Cuauhtémoc 330, Cuauhtémoc, Doctores, C.P. 06720, México, D.F., E-mail: luis.eduardo. suarez@hotmail.com

During the Breast Cancer (BC) development some changes can be observed in the adipose tissue microstructure which leads to its own full destruction. Moreover, it is well known that obesity is a risk factor on BC development. Therefore an evaluation which includes the morphologic analysis on cancer-related adipocytes and the patient's nutrition stage (PNS) is important in order to understand how microstructural changes occur in the breast adipose tissue during the $\mathrm{BC}$ stages and between $\mathrm{BC}$ types. For this purpose, image analysis (IA) was used to determine morphometric parameters on cancer-related adipocytes in human tissue. Human tissue used for IA was adipose tissue from BC patients enrolled at National Medic Center Century XXI (NMCCXXI), Mexico. Complete medical diagnose for each patient was determined in the NMCCXXI. For IA, images of the adipose tissue were acquired by means of light microscopy. The captured images were analyzed through the software Fiji. The IA consisted in the segmentation of the images in order to achieve a segmentation of reasonable quality. Descriptive Statistics and an ANOVA were used for results comparison. Statistical analysis showed a logarithmic distribution of results. Results showed that healthy adipocytes have a larger size than the ones near to cancer cells. With regard to the shape, results showed that healthy adipocytes are larger, oval, polygonal and slight rough, while the adipocytes near to the cancer regions have a smaller size, its shape is more circular and are rougher than the healthy ones, which suggests that adipocytes are involved in tumorigenesis and metastasis processes. Finally, it can be concluded that IA was useful to prove that there is a morphometric difference between healthy and cancer cells. Future work will analyze PNS and will provide more information between BC and PNS.
P104. Relationship between prevalence of metabolic syndrome and dietary patterns in obese Mexican population

Madrigal-Acevedo $\mathrm{J}^{{ }^{*}}$, Castro-Reyes $\mathrm{MA}^{2}$, Chanona-Pérez $\mathrm{JJ}^{1}$ and Valdivia-Flores $\mathbf{A}^{2}$

${ }^{1}$ Departamento de Ingeniería Bioquímica, Escuela Nacional de Ciencias Biológicas, Instituto Politécnico Nacional, Mexico

${ }^{2}$ Especialidad en Manejo Nutricional de la Obesidad y el Síndrome Metabólico del Centro Interdisciplinario de Ciencias de la Salud, Unidad Milpa Alta, Instituto Politécnico Nacional, Mexico, E-mail: jocelynmadrigal580@yahoo.com.mx

In Mexico the prevalence of obesity in young people has been increasing in the last 10 years, and the presence of this pathology is a risk for developing Metabolic Syndrome (MS). Data from 2012 showed that around $70 \%$ of the Mexican population over 20 years have overweight and obesity, moreover in the country has a prevalence of MS of $59.7 \%$ in obese people (1). Based on this, a study was conducted on the university students with Body Mass Index (BMI) from 30 to 39.9. The study evaluated the prevalence of MS with reference to the diagnostic criteria of the International Diabetes Federation (IDF) and additionally analyzed the frequency of food consumption was assessed by applying the Consumption Frequency Questionnaire (CFQ), which is an instrument designed by the Centro de Salud en Investigación Poblacional of National Public Health Institute (Mexico). For data analysis, descriptive statistics and principal component analysis (PCA) were employment. The results showed that, the prevalence of MS considering three or more IDF criteria for diagnosis of study population was $61.7 \%$, furthermore with regard to CFQ three different dietary patterns were found, were it was highlighted for all of them a high consumption of foods rich in carbohydrates such as beans and corn tortilla and as well as a high consumption of meat, fat, sugar and low consumption of fruits and vegetables, which is expected for obese Mexican population (2). Finally it can be concluded that obesity, and even MS are related to the type of food, which is consistent with literature (3). Future work will be focused on a comparison between dietary patterns and prevalence of MS in both obese and normal weight populations.

P105. Association of metabolic syndrome with the cardioankle vascular index in asymptomatic Korean population

\section{Nam SH ${ }^{1^{*}}$, Kang SG ${ }^{1}$ and Song SW ${ }^{1,2}$}

${ }^{1}$ Department of Family Medicine, St. Vincentss Hospital, College of Medicine, The Catholic University of Korea, Suwon, Republic of Korea

${ }^{2}$ Health Promotion Center, St. Vincentss Hospital, College of Medicine, The Catholic University of Korea, Suwon, Republic of Korea, E-mail: shnam1130@naver.com

Aim: Metabolic syndrome is characterized by a cluster of atherosclerotic cardiovascular risk factors. The Cardio-Ankle Vascular Index (CAVI) reflects arterial stiffness and may be used as an indicator of atherosclerotic cardiovascular disease. In this study, we investigated the association of CAVI with metabolic syndrome. Methods: A total of 1,144 adults were included in this study. We measured CAVIs and examined blood samples to identify metabolic syndrome according to WHO Asia-Pacific criteria and NCEP-ATPIII criteria. AST, ALT, r-GTP, BUN, creatinine, high sensitivity C-reactive protein and uric acid were also measured.

Results: CAVI values were significantly higher in subjects 
with metabolic syndrome than those without metabolic syndrome, and increased according to the number of metabolic syndrome components present. Subjects with high fasting blood sugar levels or high blood pressure showed high CAVI values. Multiple regression analysis showed that age, sex, diastolic blood pressure, and uric acid were independent predictors of CAVI.

Conclusion: Subjects with metabolic syndrome had high CAVIs, which indicated arterial stiffness and were closely associated with an increase in the number of metabolic risk factors. The individual risk factors for metabolic syndrome have the synergistic effect of elevating arterial stiffness in asymptomatic Korean population.

\section{P106. Ultrasensitive detection of uranyl ion in natural water using gold nanowire combined dnazyme sensor}

\section{Gwak R*, Kim H and Kim B}

Korea Advanced Institute of Science and Technology, E-mail: mushad@kaist.ac.kr

Uranium is a one of essential raw material in nuclear energy generation, however there is also concern about severe damages to human bodies and natural environments. Here, we report ultrasensitive and high selective uranyl ion detection method in natural water by plasmonic nanowire interstice (PNI) sensor combined with DNAzyme cleaved reaction. The DNAzyme-substrate which includes raman active molecules is selectively cleaved by uranyl ion, as a consequence, the released part of substrate strand is captured by PNI sensor modified with complementary DNA and then finally we could observe that SERS signal is turned on. Combining the high sensitivity of the PNI sensor and DNAzyme cleaved reaction significantly improves uranyl ion detection performance, resulting in the detection limit $1 \mathrm{pM}$ and also showed high selectivity for target uranyl ion. Furthermore, the PNI sensor excellently worked in uranium-contaminated natural water samples, which showed the potential application of this sensor for practical uranyl ion detection. Therefore, we expect that not only uranyl ion but also various toxic metal ions can be detected with high sensitivity and selectivity by applying various ion specific DNA base ligands to this PNI sensor.

P107. Ultraselective targeting from well-ordered antibodies on ultraflat single-crystalline au nanoplates for attomolar detection of c-reactive protein

\section{Lee $\mathrm{MY}^{\star}$ and Kim B}

Department of Chemistry, Korea Advanced Institute of Science and Technology, Daejeon305-701,South Korea, E-mail: leemiyeon@kaist. ac.kr

Because cardiovascular diseases induce serious complications and require continuous treatments after onset, their prophylaxis through early-diagnosis is highly important. The C-reactive protein (CRP) is acute phase reactant which is formed in body with inflammation, having been reported widely as a prognostic marker of cardiovascular diseases. For the ultrasensitive CRP detection, we combined cysteine tagged protein $G$ which immobilizes anti-CRPs with correct orientations to an ultraflat, ultraclean and single-crystalline $\mathrm{Au}$ nanoplates through Au-S bonding. Especially, atomically smooth single-crystalline $\mathrm{Au}$ nanomaterials is very suitable to fabricate reproducible sensitive sensors. And we confirmed a high surface coverage of anti-CRPs supported by protein $\mathrm{G}$ on the Au nanoplates from atomic force microscopy measurement. Interestingly, the well- ordered anti-CRPs by combination of the Au nanoplate and protein $G$ show the great selectivity toward non-target compounds at their concentration commonly found in blood. Moreover, surface enhanced Raman scattering sensor prepared by assembly of [Au nanoplate + protein G] complex and Au nanoparticles can detect CRP with 1 $\mathrm{aM}$ of detection limit. The ultraflat $\mathrm{Au}$ nanoplate might be the best substrate to immobilize protein $\mathrm{G}$ without negative effects caused by surface roughness. We expect that the detection platform employing the complex of single-crystalline Au nanoplates and protein G can be used in various high-sensitive biosensor applications which employ the IgG typed antibodies.

P108. Relationship between nutrition, inflammatory bowel diseases and anemia

\section{Yoldas $\mathrm{H}^{\star}$, Hizli $\mathrm{H}$ and Togay S}

Department of Nutrition and Dietetics, School of Health Science, Istanbul Medipol University, Turkey,E-mail: hyoldas@medipol.edu.tr

Inflammatory bowel diseases (IBD) comprise two main distinct clinical entities: Crohn $>$ s disease (CD) and ulcerative colitis (UC). These diseases occur in genetically susceptible individuals, influence gastrointestinal system is generally idiopatic. Anemia, a frequent systemic complication in patients with inflammatory bowel disease (IBD), has a complex and multifactorial pathogenesis (inflammation, iron, B12 and folic acid deficiency, myelosuppression). Etiology of anemia of chronic disease in IBD is corruption at different levels of erythropoiesis. Anemia of chronic disease occurs when iron formed after destruction of erythrocytes is stored/held in macrophages and dendritic cells in the reticuloendothelial system (RES) instead of being transferred to the erythroid progenitor cells. All these mechanisms are «functional iron deficiency» causes. Although in this case the iron in the body is not available for erythropoiesis. It was reported that presence of chronic inflammation in the body and negative effects of the drugs used in the treatment cause this situation. Recently there is a significant increase in prevalence of IBD but although many experimental studies have been performed, no agent could be found providing a complete cure in clinical practice. Treatment of IBD is mostly medical; there isn't any evidence based protocol for medical nutrition therapy. Human microbiota is a complex structure consisting of bacteria, virus, fungi and protozoa. Especially intestinal microbiota hosts 100 trillion microorganisms and it is a complex microbial community which plays an important role in immune system. Some of these microorganisms have probiotic features. Probiotics are living organisms that provide benefits to the health of the host when consumed in adequate amounts. Mostly we get probiotics from the foods in our diet and they are colonized especially in the gut. Although Lactobacillus and Bifidobacterium species are the most commonly seen probiotic microorganisms, Escherichia coli Nissle, Enterococs, some Bacillus species, Saccharomyces boulardii and some strains of Saccharomyces cerevisiae (baker's yeast) may show probiotic features. Fermented products especially contain lactic acid producing lactic acid bacteria (LAB). In this large familiy mostly Lactobacillus, Streptococcus, Enterococcus, Lactococcus, Bifidobacterium, and Leuconostoc exist. All LAB (except for Lactobacillus bulgaricus and Streptococcus thermophilus) are members of the gut flora. Thus, the treatment of these diseases, nutritional therapy are recommended as the search for alternative treatment methods. Bacteria found in the flora, foods contribute to the improvement of our health by positively affecting the gut microbiota. These microroganisms, old and forgotten precious friends traditionally found in our foods and useful for our 
body, are gaining importance day by day and their place in literature is increasingly improving.

\section{P109. The influence of $\alpha$ - lipoic acid on selected biochemical parameters in rats' livers exposed to sodium fluoride}

Grzegorzak $\mathrm{N}^{1^{*}}$, Stawiarska- Pięta $\mathrm{B}^{1}$, Janiga $\mathrm{B}^{1}$, Baczyńska $\mathrm{K}^{1}$, Zalejska- Fiolka $\mathrm{J}^{2}$ and Birkner $\mathrm{E}^{2}$

${ }^{1}$ Department of Pathology, School of Pharmacy, Division of Laboratory Medicine in Sosnowiec, Medical University of Silesia, Katowice, Poland

${ }^{2}$ Department of General Biochemistry, School of Medicine, Division of Dentistry in Zabrze, Medical University of Silesia, Katowice, Poland, E-mail:bpieta@sum.edu.pl,grzegorzak.natalia@gmail.com

Nowadays person's exposure to water containing fluoride concentrations which exceeds the limit values is a big issue because it relates to areas inhabited by large populations. It contributes to skeletal and dental fluorosis. Fluoride affects the activity of enzymes interfering with the metabolism of lipids, proteins and carbohydrates. It generates oxidative stress which contributes to the severity of the damage to the cells. The aim of the study was to evaluate the effect of a diet supplementation with $\alpha$ - lipoic acid on the biochemical parameters the rat's liver after the fluorine intoxication. The study was conducted on 18 Wistar rats. The rats were divided into 3 groups. The animals in the control group were given distilled water. Rats from the studied groups (1st, 2nd) were given $\mathrm{NaF}(2,5 \mathrm{mg} / \mathrm{rat} / 24 \mathrm{~h})$. Additionally, rats from the 2 nd group were administered $\alpha$ - lipoic acid $(17,5 \mathrm{mg} / \mathrm{rat} / 24 \mathrm{~h})$. The experiment lasted 5 weeks. To biochemical examination, one needed collected some fragments of livers. $10 \%$ of these parts served as homogenates in which the activity of enzymes was determined: alkaline phosphatase (ALP) and leucyloaminopeptidase (LAP). What is more, the concentrations of urea, proteins and FRAP were evaluated. Sodium fluoride influenced significantly the tested parameters. The tendency has been shown toward significant increase in ALP activity and a significant decrease in the activity of LAP in case of the group exposed to NaF. There was also a significant decrease in urea concentration and a FRAP. Administration of antioxidant resulted in significant increase in activity of LAP and serum urea as compared to the corresponding value observed in Group I (NaF). $\alpha$ - lipoic acid has not affected the ALP activity and the concentration of FRAP. The results show the positive, but not fully effective, influence of $\alpha$ - lipoic acid on the changes caused by $\mathrm{NaF}$ in rats'liver.

P110. The influence of antioxidants on the morphology of the thymus and adrenal glands of rats intoxicated with sodium fluoride

Stawiarska-Pięta $B^{1^{*}}$, Janiga $B^{1}$, Grzegorzak $N^{1}$, Dziurdzikowska $K^{1}$, Zalejska- Fiolka $\mathrm{J}^{2}$ and Birkner $\mathrm{E}^{2}$

${ }^{1}$ Department of Pathology, Faculty of Pharmacy in Sosnowiec, Medical University of Silesia in Katowice, Poniatowskiego 15, 40-055 Katowice, Poland

${ }^{2}$ Department of General Biochemistry, Faculty of Medicine in Zabrze, Medical University of Silesia in Katowice, Poniatowskiego 15, 40-055 Katowice, Poland, E-mail: bpieta@sum.edu.pl

In recent years, studies are being conducted on the effect of antioxidants on the biochemical and morphological changes of induced fluorine within the different organs. They are related to the demonstrated influence of fluorine on oxidation-reduction system, especially on the activity of antioxidant enzymes. The aim of the study was to examine the influence of antioxidants on morphological picture of the adrenal glands and the thymus of rats exposed to sodium fluoride. The study was performed on $24 \mathrm{Wistar}$ rats. The animals were divided into 4 groups. Rats from control group were given distilled water. Rats from the studied groups (1st, 2nd and 3rd) were given $\mathrm{NaF}(2,5$ $\mathrm{mg} / \mathrm{rat} / 24 \mathrm{~h}$ ). Additionally, rats from the 2 nd group were given $\mathrm{CoQ}$ $(200 \mathrm{mg} / \mathrm{rat} / 24 \mathrm{~h})$. In the $3 \mathrm{rd}$ group the animals were given vitamin $\mathrm{E}$ (3 mg/rat/24h), vitamin A (250 IU/rat/24h) and CoQ in the dose as before. The experiment lasted for 7 weeks. Upon dissection the thymus and the adrenal glands were taken for histopathological examination. The pathomorphological changes in the organs were assessed with preparations obtained with the normal paraffin method, stained with hematoxilin and eosin. Morphological changes were assessed in the light of Olimpus microscope. In the studied thymuses, as well as in the adrenal glands of rats exposed to sodium fluoride retrogressive changes have been shown. Besides, the erythrorrhagia and hyperemia were also revealed. The results show the positive, but not fully effective, influence of CoQ on the changes caused by fluoride in rats' organs. The use of a combination of antioxidants: CoQ and vitamins $\mathrm{A}$ and $\mathrm{E}$ has occurred to be fully effective in protecting in respect of the thymus adrenal glands. Conducted research indicates the possibility of prevention from effects of exposure to fluoride through antioxidant dietary supplement.

P111. The effect of nutrition and food consumption on the size of the ecological footprint in somogy county (Hungary)

\section{Lelovics $\mathrm{Zs}^{\star}$ and Tossenberger J}

Kaposvár University, Faculty of Agricultural and Environmental Sciences, Hungary,E-mail: lelovics@yahoo.com

Aims: The ecological footprint represents the extent of our environmental impact. With the help of the concept the effect of our activities on nature can be measured and demonstrated. The authors aimed to define Somogy County's population's ecological footprint focusing on food consumption and nutrition.

Materials and Methods: The authors conducted an anonymous, voluntary and self-administered ecological footprint survey with participants over 18 years $(n=493,41 \%$ men and $59 \%$ female, mean age $38+/-9$ years) based on the Hungarian version of Eric Krausess calculation method known as the Association for Environmental Management. The term relates to the value of energy and material used by human activity in an area of a hectare. The calculation defines the required area of land and water surface. The data analysis was performed by comparing $95 \%$ confidence intervals and two-sample t-tests.

Results: When calculating the ecological footprint, the average scores were $366+/-72,6,0-7,8$ hectares respectively, while taking into account the population and area of the Earth, 1.8 hectares are available per individuals. If the entire population of Earth would lead a life similar to the young and middle-aged population living in Somogy County there should be three planets like Earth to serve them. The authors give a detailed presentation of the results in their lecture.

Conclusions: The ecological footprint gives an expressive aspect of the environmental strain, and with the concept the measurement and the presentation of our effect on nature became possible. Based on the answers of the questioned individuals from Somogy County, it can be stated that they live in great style. Nutrition and food preparing form only one feature of the desired sustainable consumption behavioural attitudes. The authors' results, correlating with the literature, show that 
consuming organic food contributes to the sustainable development. One quarter of the scores concerning nutrition attitudes could be earned with the frequency of meat and fish consumption. Even some of the participants, among those who do not consume meat or fish are not concerned with the origin of the food, and do not pay attention to choose home-made food rather than ready made products either. The questionnaire helps to differentiate those who do not consume meat in order to protect the environment, yet they might have other possibilities to do so. On one hand, with analysing the ecological footprint questionnaire the environmental strain is measurable, and on the other hand it is possible to emphasize the related nutritional attitudes and interrelations.

\section{P112. Screening of nutrition and nutritional status of marfan syndrome patients}

\section{Kiss $\mathbf{A}^{1^{*}}$ and Lelovics $\mathrm{Zs}^{2}$}

${ }^{1}$ Faculty of Food Science, Corvinus University of Budapest, Budapest, Hungary

${ }^{2}$ Kaposvár University, Kaposvár, Hungary, E-mail: kiss.anna891@ gmail.com

Aims: Marfan syndrome is a genetic disorder of the connective tissue, which affects approximately 2000-3000 individuals in Hungary. Given its multi-systemic manifestations, this disorder is often difficult to diagnose. To date, the National Marfan Register system contains approximately 250 cases, and this number is dynamically increasing. Our goal is to assess the nutrition and nutritional status of Hungarian patients suffering from Marfan syndrome.

Methods: We screened in 2014 the nutritional status of 114 voluntary Marfan-syndrome patient elder than 18 years (50\% male, $50 \%$ female) with the validated Malnutrition Universal Screening Tool (MUST), and with the Food Frequency Questionnaire for valid nutritional status assessment.

Results of the research: According to our results the risk of malnutrition for these people is high (25.5\%), but the prevalence of the malnutrition depends on the assessed and number of those parameters. The proportion of malnutrition among people according to their Body Mass Index is $12.3 \%$, and according to their weight loss is also $8.8 \%$. We found that the effect of acute illnesses on the malnutrition risk is $10.5 \%$.

Conclusions: Based on the reference of the European Union, national health care systems need to attend particularly to the recognition of rare diseases, and to the development of patient treatment. The screening of nutrition and nutritional status of Marfan syndrome patients also provides new opportunities to study Hungarian patients with Marfan syndrome to reduce the prevalence of malnutrition important to screen the nutritional status.

P113. Utilization of solid wastes of food industry to produce biocatalysts for fermentations at extremely low temperatures

Dimitrellou D*, Ganatsios V, Bekatorou A, Koutinas A and Kanellaki M

Department of Chemistry, Food Biotechnology Group, University of Patras, 26500 Patras, Greece, E-mail: dimitrellou@gmail.com

Low temperature fermentation was always a challenge for food industry due to numerous advantages compared to traditional fermentations resulting to products with improved flavor and nutritional value. In addition the production of new biocatalysts suitable for applications in such low temperatures is also a necessity. Nanotechnology, the last years, has spread its applications also in food sector establishing great potentiall. Especially nano-tubular cellulose technology is gaining attention for several applications for food production2. The aim of the present study was to evaluate the use of solid wastes of food industry as supports for the development of new biocatalysts suitable for applications at low temperature fermentations in foods. Therefore solid wastes of food industry such as brewers' spent grains, wheat bran, peanut and coconut shells were subjected to delignification with $\mathrm{NaOH}$ solution. After production, the tubular cellulose from all origins was used as support for cell (Saccharomyces cerevisiae or Lactobacillus casei) immobilization. The immobilized biocatalysts were subjected to thermal drying with air circulation at $38 \mathrm{oC}$ for 24 hours and then were stored at $5 \mathrm{oC}$ for up to three months, in order to make them more compatible with the food industry need for dried and ready to use biocatalysts. After storage the thermal dried biocatalysts were used for beer (Saccharomyces cerevisiae) and fermented milk (Lactobacillus casei) production. Fermentation kinetics were performed at each temperature and the main characteristics of the produced beer and fermented milk were measured. The results confirmed the positive effect of immobilization on the characteristics of the produced fermented products and the suitability of these new biocatalysts for low temperature fermentations in food industry. The results of the present study are very promising for possible applications in food industry sector using low cost food industry wastes as supports for new biocatalysts production for numerous applications in food production.

P114. Association of household composition with healthy food intake and obesity among us adults

\section{Sung $\mathrm{JH}^{1^{*}}$, Lee $\mathrm{JY}^{2}$ and Lee $\mathrm{JE}^{1}$}

${ }^{1}$ Jackson State University, USA

${ }^{2}$ University of Miami,USA,E-mail: jung.h.lee@jsums.edu

Background: Promoting healthy eating is important in preventing obesity which is an imminent critical health threat, with high likelihood for multiple co-morbidities as cardiovascular disease and hypertension, renal failure, and nutrition imbalance. Household members formulate/share the common norm and values on eating behavior through frequent interaction among members. Therefore, household composition may be an important factor in understanding the eating pattern and epidemic episode of obesity.

Objective: This study is to investigate the association of household composition with eating pattern and obesity among US adults.

Methods: A total of 432,607 adults from the BRFSS data were included in the analyses. Healthy eating was defined as daily consumption of 5 or more servings of fruits and vegetables. Household composition is defined as 6 varied sets of household members of adult women, adult men and children. A series of SURVEYLOGISTIC analyses controlling for major demographics were conducted to determine the association of household composition with eating pattern.

Results: While a higher prevalence of consuming 5 or more servings of fruits and vegetables (f/v) daily was reported among those in household of adult women only $(27.6 \%)$ and adult women + adult men (25.0\%), a lowest prevalence was reported among those in household 
without adult women (adult men only, 18.6\% and adult men + children, 18.6\%). This pattern differed by marital status, race, educational level and age. Unmarried adults living in single family household (24.7\%) consumed v/f more than those living in multi-family household (22.0\%). A multivariable SURVEYLOGISTIC analysis including three household components revealed that while number of adult men in the household $(\mathrm{p}=0.0002)$ was negatively associated with $\mathrm{f} / \mathrm{v}$ intake, number of adult women $(\mathrm{p}<0.0001)$ and number of children $(\mathrm{p}=0.0090)$ were positively associated with it. Another SURVEYLOGISTIC regression demonstrated that while less $\mathrm{f} / \mathrm{v}$ intake and less adult men was linked to higher obesity, more children and more adult women were associated with higher obesity.

Conclusions: Fruit and vegetable intake was the lowest among adults living in the household without adult women. Our multivariable analysis also suggested that number of adult women was the most important component in consuming fruit and vegetables, implying adult women may be key target in promoting family healthy eating.

\section{P115. Study of the effect of thymus vulgaris and curcuma} longa on diabetic rats

\section{Khafagy $\mathrm{M}^{\star}$ and Abd El-Rahman AN}

Department of Nutrition and Food Science, Faculty of Home Economics, Minoufiya University, Shebin El-kom, Egypt, E-mail: maikhafagy46@yahoo.com

This study was conduced to investigate the effect of Thymus vulgaris and Curcuma longa on Diabetic Rats. Thirty six mature albino rats weighting 150-160g B.w.\% each were used, and divided into 6 equal groups, one was kept as a control-ve group, while the other groups were made diabetes by Aloxan $(150 \mathrm{mg} / \mathrm{kg})$. The tested plants were given as a percent of $5 \%$ and $10 \%$ from the Basel diet for four weeks. After the treatment, blood samples were taken and serum glucose, triglyceride, total cholesterol, HDL-C, LDL-C, VLDL levels were measured, and (F.I, FER, BWG\%) and histopathological changes of liver and kidney were examined. The results indicated that rats treated with Aloxan recorded significantly increasing in serum glucose, total cholesterol, LDL-C, VLDL and triglyceride levels $(\mathrm{p}<0.005)$ while HDL-C level was decreased $(p<0.005)$ as compared to the control group. Treatment of diabetic rats with Thymus vulgaris and Curcuma longa caused a decrease in serum glucose, total cholesterol, LDL-C, VLDL and triglyceride levels $(\mathrm{p}<0.005)$ with an increase in HDL-C level $(\mathrm{p}<0.005)$. When compared with the control group. Also, diabetic groups had histopathological changes on liver and kidney through degeneration hyperemia, inflammatory reaction. The post-treatment of tested plants leads to prevent some of the previous histopathological changes. It could be concluded that the tested plants were effective in protecting against diabetic rats not only decreased the level of serum glucose but also has beneficial effect on serum lipids. Therefore, we recommended those tested plants by a moderate amount to be included in our daily diets and drinks.

P116. Studying the effect of leaves and seeds powder of carica papaya at two doses on hyperuricemic of experimental animals

\section{Hussein $\mathrm{ES}^{1^{*}}$, Yahia $\mathrm{NM}^{2}$ and Hussein $\mathrm{EA}^{2}$}

${ }^{1}$ Department ofNutrition and Food Science, Faculty of Home Economics, Minofiya University, Egypt
${ }^{2}$ Department ofNutrition and Food Science, Faculty of Home Economics, Al-Azhar University,Egypt,E-mail: dresam_boudi@yahoo. com

Hyperuricemia is a primary risk factor for the development of gout. A Carica papaya fruit is a source of nutrients such as provitamin A carotenoids, vitamin $\mathrm{C}$, folate and dietary fiber, pulp and seeds also contain a variety of phytochemicals, including lycopene and polyphenols. The current research study the effect of seeds and leaves of Carica papaya at two different concentrations (5 and 10\%), on level of Hyperuricemia between healthy and hyperuriecemic rats. Thirty six male albino rats weight $150 \pm 10 \mathrm{~g}$ divided into six groups (+ve), (-ve), (5 and $10 \%)$ leaves and seeds powder. Hyperuricemic induced by alcohol ( $2 \mathrm{~g} / \mathrm{kg}$ of body weights every 24 hours) for six days. Food intake (FI), body weight gain and organs weight were recorded. Furthermore, Biochemical analysis of serum; urea, creatinine, uric acid, total protein, albumin, globulin, A/G ratio, AST, ALT, total cholesterol, triglyceride, HDL-c, LDL-c, and VLDL-c were determined. Both seeds and leaves caused an increase in the body weight for both used concentrations. However used leaves at 5\% raised FI between in all the studied groups. On the other hand, kidney weight shows no effects between all the groups in both leaves and seeds although there is an increase in the liver weight for both leaves and seed 10,5\%, respectively. Surprisingly, all the determined urea levels in all the studied groups decreased; however, seeds with $10 \%$ were the highest reduction among all groups. Uric acids significantly decreased at level $10 \%$ in seed powder, there were a significantly decreased in serum ALT and AST at two levels for leaves and seeds powder. Seeds powder at two doses caused a decrease in total protein and globulin while, in serum albumin was increased significantly at two levels. Leaves powder at $10 \%$ and seeds at two doses cause a decrease in serum total cholesterol and serum triglycerides. Serum HDL was significantly increased while, serum LDL was decreased. Consumption Carica papaya seeds or leaves caused improvement benefits in health status.

P117. Antioxidant activities of some extract of tamarix gallica 1. containing phenolic compounds

\section{Akkal S*, Lefaha M and Zaabat $\mathrm{N}$}

Department of Chemistry, Research Unity:Valorization of Natural Resources, Bioactive Molecules, University of Constantine 1, 25000 Constantine, Algeria, E-mail: salah4dz@yahoo.fr

The genus Tamarix L. belongs to the Tamaricaceae family. The Tamarix is found to be rich in polyphenolic compounds such as flavonoids, phenolic acids, tannins and coumarins. Several researches proved antioxidant and antimicrobial activities of some Tamarix species. In Algeria, Tamarix genus includes ten species. The present work deals with the phytochemical and biological studies as well as the antioxidant activities of methanol and ethyl acetate extracts obtained from Tamarix gallica, collected during the flowering. The phytochemical analysis of aerial parts of Tamarix gallica was undertaken. Ethyl acetate and methanol extracts led to the isolation of three known phenolic compounds: 3', 3, 5-tri hydroxy 4', 7- diméthoxy flavone(1) , 5-Hydroxy 4>,3,7-trimethoxyflavone(2) , 3, 5, 7-tri hydroxy 4'- méthoxy flavone(3) and isorhamnetine(4). The structures of these compounds were elucidated by MS and a series of $1 \mathrm{D}$ and 2D NMR analyses. The antioxidant activities of some extracts and the pure isolated compounds (1-3) were determined by two different analytical methods: cupric reducing antioxidant capacity (CUPRAC) and 2, 2'-diphenyl-1-picrylhydrazyl (DPPH). 
P118. Highly selective and sensitive optical sensor for determination of creatinine in blood and urine samples based on silver nanoparticles

\section{Khayatian $\mathrm{G}^{\star}$ and Mohammadi S}

Department of Chemistry, University of Kurdistan, Sanandaj, Iran, E-mail:gkhayatian@uok.ac.ir

Creatinine (2-amino-1-methyl-5H-imidazol-4-one) is one of the most common biomarkers and the end metabolic product of the creatine in mammals. Determination of creatinine concentration in biological fluids is important in the evaluation of renal function and muscle damage [1, 2]. The normal physiological creatinine concentrations in serum are in range of 35 to $140 \mu \mathrm{M}$. The increased creatinine concentration leads to diabetic nephropathy, preeclampsia, glomerulonephritis, urinary tract obstruction and renal failure, while the decreased creatinine concentration leads to muscular dystrophy and myasthenia. Therefore, the determination of creatinine in body human plasma or urine is of great importance from the biological and pharmacological stand points [1]. In this study, a simple, fast, selective and sensitive colorimetric method was developed for the determination of the highly important diagnostic paramater creatinine. Silver nanoparticles were capped with 2,2>-thiodiacetic acid, and it is found that creatinine causes the aggregation of these AgNPs, while other biomolecules do not have an effect. As a result, the color of the solution changes from yellow to green which can be detected visually or by spectrophotometry. The aggregation of the AgNPs was confirmed by UV-vis absorptiometry and transmission electron microscopy. This colorimetric probe allows creatinine to be rapidly quantified with a 3.0 $\mathrm{nM}$ limit of detection. The ratio of absorptions at $560 \mathrm{~nm}$ and $390 \mathrm{~nm}$ is linearly correlated to the concentration of creatinine in the $0.01 \mu \mathrm{M}$ to $1.0 \mu \mathrm{M}$ range. The method was successfully applied to the determination of creatinine in (spiked) human serum plasma and urine samples and gave recoveries that ranged from 89.4 to $102.1 \%$. The method was also validated by using the Jaffe standard method.

\section{Author Index}

$\begin{array}{ll}\text { Akkal S } & \text { A64 } \\ \text { Aljumayi H } & \text { A21 } \\ \text { Amoako K } & \text { A40 } \\ \text { An ES } & \text { A44 } \\ \text { Ancillotti C } & \text { A15 } \\ \text { Artene D } & \text { A54 } \\ \text { Aruwa S } & \text { A12 } \\ \text { Asanuma N } & \text { A26 } \\ \text { Athauda LK } & \text { A18 } \\ \text { Benedec D } & \text { A24 } \\ \text { Betz JA12 } & \\ \text { Bingol EB } & \text { A35 } \\ \text { Bondarenko I } & \text { A07 } \\ \text { Brasacchio C } & \text { A16 } \\ \text { Buchler T } & \text { A14 } \\ \text { Buyukuslu N } & \text { A43 }\end{array}$

\begin{tabular}{|c|c|}
\hline Colak H & A35 \\
\hline Chang E & A 37 \\
\hline Choi YK & A51 \\
\hline Choi YY & A41 \\
\hline Deniz S & A47 \\
\hline Devar S & A22 \\
\hline Dieleman L & $\mathrm{A} 07$ \\
\hline Dimitrellou D & A63 \\
\hline Dostálek P & A09 \\
\hline Dumlu G & A48 \\
\hline Fibigr J & A28 \\
\hline Gorska S & A06 \\
\hline Grzegorzak N & A61 \\
\hline Gülden G & $\mathrm{A} 17, \mathrm{~A} 54$ \\
\hline Gwak R & A60 \\
\hline Hamouda A & $\mathrm{A} 20, \mathrm{~A} 21$ \\
\hline Han S & A43 \\
\hline Hasegawa M & A38 \\
\hline Heo EJ & A54, A55 \\
\hline Hildreth J & A13, A53 \\
\hline Hizli H & A26 \\
\hline Hong SH & A52 \\
\hline Horna A & A11 \\
\hline Huh CS & A51 \\
\hline Hussein E & A64 \\
\hline Iwamoto A & A31 \\
\hline Jadavji N & $\mathrm{A} 14$ \\
\hline Jandera P & $\mathrm{A} 10$ \\
\hline Jang YH & $\mathrm{A} 30$ \\
\hline Jansen E & $\mathrm{A} 03, \mathrm{~A} 13, \mathrm{~A} 53$ \\
\hline Jansom C & A20 \\
\hline Jansom V & $\mathrm{A} 20$ \\
\hline Jeung W & A32 \\
\hline Jirillo E & A06 \\
\hline Jung $\mathrm{H}$ & A36 \\
\hline Jung S & A29 \\
\hline Kadri F & A55 \\
\hline Kameya H & $\mathrm{A} 24$ \\
\hline Kato N & A18 \\
\hline Khafagy M & A64 \\
\hline
\end{tabular}




\begin{tabular}{|c|c|c|c|c|}
\hline Khayatian G & A65 & Nanasombat S & A21 & \\
\hline Kim AJ & A52 & Neunghye L & A29 & \\
\hline Kim DH & A49 & Ngoh YY & A46 & \\
\hline Kim H & A09, A23 & Oyong G & $\mathrm{A} 10$ & \\
\hline Kim K & A41 & Özden Tuncer B & & A33 \\
\hline Kim MH & A41 & Ozpak Akkus O & A49 & \\
\hline Kim MJ & A43 & Pavicic Zezelj S & A29 & \\
\hline Kim NY & A 37 & Pinchuk I & A39 & \\
\hline Kim SJ & A25 & Piwoni A & A34 & \\
\hline Kim YJ & A50 & Russell M & A03 & \\
\hline Kiran M & A 48 & Ryu R & A50 & \\
\hline Kiss A & A62 & Saka M & A56 & \\
\hline Kiziltan G & A57 & Saphier O & A39 & \\
\hline Koren O & A03 & Semenova VN & $\mathrm{A} 30$ & \\
\hline Krejčí J & A18 & Sevinc E & A23 & \\
\hline Krych-Madej J & A 44 & Schiefer HP & A19 & \\
\hline $\mathrm{Ku} \mathrm{JM}$ & A52 & Siow HL & A46 & \\
\hline Lee AS & A44 & Smetanova $\mathrm{H}$ & A49 & \\
\hline Lee MY & A60 & Son $\mathrm{H}$ & A58 & \\
\hline Lee MJ & A 37 & Stasiuk M & $\mathrm{A} 22$ & \\
\hline Lee S A36 & & Stawiarska-Pięt & ta B & A61 \\
\hline Lee WK & A24 & Stepankova R & A08 & \\
\hline \multicolumn{2}{|l|}{ LeeSJ A17, A38 } & \multicolumn{2}{|c|}{ Suárez-Nájera LE } & A58 \\
\hline Lelovics Zs & A62 & Sung JH & A63 & \\
\hline Lichen Y & A05 & Szeiffova Bacova & $\mathrm{a} B$ & A27 \\
\hline Mabrouk RR & A13 & Táborský J & A42 & \\
\hline Macka M & A11 & Takahara K & A32 & \\
\hline \multicolumn{2}{|c|}{ Madrigal-Acevedo J } & Tan SJ & A45 & \\
\hline Mach I & A15 & Tiperciuc B & A30 & \\
\hline \multicolumn{2}{|c|}{ Machackova M A46 } & \multicolumn{2}{|c|}{ Tlaskalová-Hogenová H } & A08 \\
\hline Marsh A & A05 & Tribulova N & $\mathrm{A} 27$ & \\
\hline Meklat A & A55 & Tuncer Y & A34 & \\
\hline Mestecky J & A03 & Turker PF & A57 & \\
\hline Monro J & A04 & Ukai M & $\mathrm{A} 23$ & \\
\hline Moustafa $\mathrm{H}$ & A 22 & Um M & A33 & \\
\hline Mucha M & A 12 & Viczenczová C & A26 & \\
\hline Mulders, MDGH & A47 & Vieira EF & A16, A45 & \\
\hline Muráriková A & $\mathrm{A} 40$ & Viezeliene D & A53 & \\
\hline Nakagawa Y & A35 & Vodicka P & A04 & \\
\hline Nam SH & A59 & Voráčová J & A47 & \\
\hline
\end{tabular}




$\begin{array}{llll}\text { Walsh CM } & \text { A25 } & \text { Yang WM } & \text { A40 } \\ \text { Woo SM } & \text { A51 } & \text { Yoldas H } & \text { A60 } \\ \text { Yang HJ } & \text { A39 } & \text { Zaki S } & \text { A36 }\end{array}$

Copyright: ( 02016 Horna A. This is an open-access article distributed under the terms of the Creative Commons Attribution License, which permits unrestricted use, distribution, and reproduction in any medium, provided the original author and source are credited. 